\title{
Analysis of Stability and Dispersion in a Finite Element Method for Debye and Lorentz Dispersive Media
}

\author{
H. T. Banks, ${ }^{*}$ V. A. Bokil,$^{\dagger}$ and N. L. Gibson ${ }^{\ddagger}$ \\ Center for Research in Scientific Computation \\ North Carolina State University \\ Raleigh, N.C. 27695-8205
}

August 22, 2006

\begin{abstract}
:
We study the stability properties of, and the phase error present in, a finite element scheme for Maxwell's equations coupled with Debye or Lorentz polarization. In one dimension we consider a second order formulation for the electric field with an ordinary differential equation for the polarization added as an auxiliary constraint. The finite element method uses linear finite elements in space for the electric field as well as the polarization, and a theta scheme for the time discretization. Numerical experiments suggest the method is unconditionally stable for both Debye and Lorentz models. We compare the stability and phase error properties of the method presented here with those of finite difference methods that have been analyzed in the literature. We also conduct numerical simulations that verify the stability and dispersion properties of the scheme.
\end{abstract}

Keywords: Maxwell's Equations, Debye, Lorentz, Finite Elements, FDTD, dissipation, dispersion.

*email: htbanks@ncsu.edu

†email: vabokil@ncsu.edu

${ }^{\ddagger}$ email: ngibson@ncsu.edu 


\section{Introduction}

Noninvasive interrogation of the interior of tissues and other materials by electromagnetic waves has important applications in various fields including medical imaging for the early detection of anomalies and nondestructive damage detection in aircraft $[2,3]$. For example, microwave imaging for breast cancer detection is expected to be safe for the patient and has the potential to detect very small cancerous tumors in the breast [16]. This ability for detection is based on the difference in electrical properties of malignant and normal tissues. Biological tissue interactions with the fields are defined by their complex permittivity which is a function of the various electric and magnetic polarization mechanisms and conductivity of the biological medium. Similarly, nondestructive damage detection in materials for the detection of defects such as cracks is based upon the changes in the electrical properties that occur due to the presence of these defects. Thus, one of the aims of electromagnetic interrogation problems is the determination of the dielectric properties of the materials under investigation.

To computationally simulate these electromagnetic interrogation problems requires setting up a suitable inverse problem which involves numerous forward simulations of the propagation of transient electromagetic waves in lossy dispersive dielectrics such as biological tissue. Hence, the development of accurate, consistent and stable discrete forward solvers is very important, as errors in the numerical solvers can result in inaccurate determination of the dielectric properties that determine the characteristics of the material being investigated.

The electric and magnetic fields inside a material are governed by the macroscopic Maxwell's equations along with constitutive laws that account for the response of the material to the electromagnetic field. In special cases Maxwell's equations can be reduced to a vector wave equation in the electric or magnetic fields. Numerical approximation algorithms of time-dependent wave equations and Maxwell's equations introduce error into the amplitude and speed of the propagating waves. These errors include dissipation, the dampening of some frequency modes, and dispersion, the frequency dependence of the phase velocity of numerical wave modes in the computational grid.

Dielectric materials have actual physical dispersion. The complex electric permittivity of a dielectric medium is frequency dependent (has dielectric dispersion). Thus, an appropriate discretization method should have a numerical dispersion that matches the model dispersion as closely as possible. Dielectric materials also have physical dissipation, or attenuation, which must also be correctly computed by a numerical method. In particular, if a method does not sufficiently damp initial disturbances, possibly due to round-off error, the method can become unstable. Certain algorithms have criteria based on discretization parameters to determine when it may be unstable; these are called conditionally stable methods.

The stability and dispersion properties for the finite difference time domain (FDTD) schemes applied to Maxwell's equations in free space are well known (see [35]). Additionally, different time domain finite element methods have also been devised for the numerical approximation of Maxwell's equations in free space (see [27, 23] and the references therein). While free space analyses for finite element and finite difference methods are well documented, stability and phase error analysis for dispersive dielectrics has been primarily focused on finite difference methods (see $[28,35]$ for standard second order methods and 
[29] for higher order schemes). The treatment for finite element methods has been limited to scalar-potential formulations to model dielectric dispersion at low frequencies ([31]), scalar Helmholtz equation ([24]), and in some cases, hybrid methods ([15]).

There are several FDTD extensions that have been developed to model electromagnetic pulse propagation in dispersive media. One technique is to add to Maxwell's equations a set of ODE's that relate the electric displacement $\vec{D}(t)$ to the electric field $\vec{E}(t)$ [18], or a set of ODE's that model the dynamic evolution of the polarization vector $\vec{P}(t)$ driven by the electric field $[20,19]$. Dielectric dispersion can be expressed in the time domain as a convolution integral involving the electric field and a causal susceptibility function. The recursive convolution method $[26,25,21]$ uses a recursive technique to update the convolution representation of the constitutive law along with the FDTD time update of Maxwell's equations. There are other methods such as the Z-transform [34, 33] and the TLM method [9] that have also been used to model pulse propagation in dispersive media. Many of these methods have been compared and analyzed for their numerical errors and stability properties $[28,36,30,10,12]$.

In this paper we study the propagation characteristics of the discretized Maxwell's equations with Debye or Lorentz polarization, using a finite element method, in terms of numerical stability and dispersion analyses. We obtain information about the expected accuracy of the method from the construction of the dispersion relation which relates the numerical wave number $k$ to the frequency $\omega$ for waves propagating in the finite element grid and then compare with the dispersion relation for the corresponding continuous model (differential equations). We compare the stability and dispersion properties of the finite element method with those of finite difference methods analyzed in [28]. Such finite element methods have been used for the electromagnetic interrogation of dielectric media with a metal backing, for the determination of dielectric properties and geometrical dimensions of the medium [5], as well as for the detection of cracks in composite materials [8]. In [1], finite element methods were used to interrogate dielectric media with acoustic waves as virtual reflectors.

We analyze one dimensional models to which we apply standard linear finite elements for the spatial discretization of the electric field and the polarization. Maxwell's equations are reduced to a wave equation in the electric field, and the constitutive law in the medium involves an ordinary differential equation that describes the dynamic evolution of the polarization driven by the electric field. The entire system is rewritten in first order form and time discretized using a theta scheme.

The numerical stability results for Debye and Lorentz media suggest that the extension of the finite element scheme for these media retain the unconditional stability property of the scheme in free space. The well known Lax-Richtmayer theorem [32] states that the convergence of consistent difference schemes to initial value problems represented by PDE's is equivalent to stability. Thus, unconditional stability is a desirable property of difference schemes as it allows the choice of the time step to be determined by the physical dimensions of the problem, such as a relaxation time. The FDTD methods, on the other hand, are conditionally stable. Since the stability condition is determined by the smallest cell size in the domain, the FDTD analysis of very fine geometric structures requires a large number of time iterations. In the finite element case unconditional stability allows us to choose the Courant number, which relates the time step to the mesh step size, to minimize the numerical phase error. 
The numerical dispersion analysis shows that for accuracy we need to resolve the shortest time scale in the problem which agrees with the result obtained in [28]. This is reflected in the fact that the time step for simulating Debye media should be chosen to be about $O\left(10^{-3}\right) \tau$, where $\tau$ is the relaxation time of the medium, whereas for Lorentz media, the time step should be chosen to be the minimum of $O\left(10^{-2}\right) \tau$ and $0\left(10^{-2}\right)\left(\frac{2 \pi}{\omega_{0}}\right)$, where $\omega_{0}$ is the resonance frequency of the medium.

We thus determine a guideline for users in which the time step is chosen to minimize the dissipation of the scheme and the Courant factor is chosen to minimize the phase error, providing good agreement between the exact and numerical complex permittivity.

\section{Model formulation}

We consider Maxwell's equations which govern the electric field $\vec{E}$ and the magnetic field $\vec{H}$ in a domain $\Omega$ from time 0 to $T$ given as

$$
\begin{aligned}
\frac{\partial \vec{D}}{\partial t}+\vec{J}-\nabla \times \vec{H} & =0 \text { in }(0, T) \times \Omega, \\
\frac{\partial \vec{B}}{\partial t}+\nabla \times \vec{E} & =0 \text { in }(0, T) \times \Omega, \\
\nabla \cdot \vec{D} & =0 \text { in }(0, T) \times \Omega, \\
\nabla \cdot \vec{B} & =0 \text { in }(0, T) \times \Omega, \\
\vec{E}(0, \vec{x}) & =0 \text { in } \Omega, \\
\vec{H}(0, \vec{x}) & =0 \text { in } \Omega .
\end{aligned}
$$

The fields $\vec{D}, \vec{B}$ are the electric and magnetic flux densities respectively. All the fields in (2.1) are functions of position $\vec{x}=(x, y, z)$ and time $t$. We have $\vec{J}=\vec{J}_{c}+\vec{J}_{s}$, where $\vec{J}_{c}$ is a conduction current density and $\vec{J}_{s}$ is the source current density. However, we will assume $\overrightarrow{J_{c}}=0$ in this paper, as we are interested in dielectrics with no free charges. Appropriate boundary conditions are added to system (2.1) to terminate the computational domain.

Constitutive relations which relate the electric and magnetic fluxes $\vec{D}, \vec{B}$ to the electric and magnetic fields $\vec{E}, \vec{H}$ are added to these equations to make the system fully determined and to describe the response of a material to the electromagnetic fields. In free space, these constitutive relations are $\vec{D}=\epsilon_{0} \vec{E}$, and $\vec{B}=\mu_{0} \vec{H}$, where $\epsilon_{0}$ and $\mu_{0}$ are the permittivity and the permeability of free space, respectively, and are constant [17]. In general there are different possible forms for these constitutive relationships. In a frequency domain formulation of Maxwell's equations, these are usually converted to linear relationships between the dependent and independent quantities with frequency dependent coefficient parameters. We will consider the case of a dielectric in which magnetic effects are negligible. Thus, within the dielectric medium we have constitutive relations that relate the flux densities $\vec{D}, \vec{B}$ to the electric and magnetic fields, respectively, as

$$
\begin{aligned}
\vec{D} & =\epsilon_{0} \vec{E}+\vec{P}, \\
\vec{B} & =\mu_{0} \vec{H}
\end{aligned}
$$


In (2.2a), the quantity $\vec{P}$ is called the macroscopic electric polarization. (A discussion of the relationship between the macroscopic polarization and the microscopic material properties leading to distributions of relaxation times and other dielectric parameters in the constiutive laws can be found in [6].) Electric polarization may be defined as the electric field induced disturbance of the charge distribution in a region. This polarization may have an instantaneous component as well as delayed effects; the latter will usually have associated time constants called relaxation times which are denoted by $\tau$. We define the instantaneous component of the polarization to be related to the electric field by means of a dielectric constant $\epsilon_{0}$ and a susceptibility $\chi$. The remainder of the electric polarization, called the relaxation polarization, is denoted as $\vec{P}_{\mathrm{R}}$. Therefore, we have

$$
\vec{P}=\vec{P}_{\mathrm{I}}+\vec{P}_{\mathrm{R}}=\epsilon_{0} \chi \vec{E}+\vec{P}_{\mathrm{R}}
$$

and hence the constitutive law (2.2a) becomes

$$
\vec{D}=\epsilon_{0} \epsilon_{r} \vec{E}+\vec{P}_{\mathrm{R}}
$$

where $\epsilon_{r}=(1+\chi)$ is the relative permittivity of the dielectric medium. We will henceforth denote $\vec{P}_{\mathrm{R}}$ by $\vec{P}$, as the instantaneous polarization will be absorbed into the dielectric constant $\epsilon_{r}$. The following section defines the equations for polarization models of interest in this paper.

\subsection{Models for Polarization}

To describe the behavior of the media's relaxation polarization $\vec{P}$, one may use ordinary differential equation models that describe microscopic polarization mechanisms such as dipole or orientational polarization (Debye), as well as ionic and electronic polarization (Lorentz), and other frequency dependent polarization mechanisms [4]. For more complex dielectric materials, a simple Debye or Lorentz polarization model is often not adequate to characterize the dispersive behaviour of the material. One can then turn to combinations of Debye, Lorentz, or even more general $n$th order mechanisms [5] as well as Cole-Cole type (fractional order derivative) models [11]. Additionally, materials may be represented by a distribution of the associated time constants or even a distribution of polarization mechanisms (see $[7,6]$ ). In this report we concentrate our analysis on single pole Debye and Lorentz polarization models.

\subsubsection{Debye Model}

The differential equation of the Debye model for orientational or dipolar polarization is given by

$$
\tau \frac{\partial \vec{P}}{\partial t}+\vec{P}=\epsilon_{0}\left(\epsilon_{s}-\epsilon_{\infty}\right) \vec{E} .
$$

Here $\epsilon_{s}$ is the static relative permittivity. The presence of instantaneous polarization is accounted for in this case by the coefficient $\epsilon_{\infty}$ in the electric flux equation. That is, $\epsilon_{r}=\epsilon_{\infty}$. The remainder of the electric polarization is seen to be a decaying exponential with relaxation parameter $\tau$, driven by the electric field, less the part included in the 
instantaneous polarization. This model was first proposed by Debye [14] to model the behaviour of materials that possess permanent dipole moments. The magnitude of the polarization term $\vec{P}$ represents the degree of alignment of these individual moments and is based on a uniformity assumption at the molecular level (see [6]). The choice of coefficients in (2.5) gives a physical interpretation to $\epsilon_{s}$ and $\epsilon_{\infty}$ as the relative permittivities of the medium in the limit of the static field and very high frequencies, respectively. In the static case, we have $\vec{P}_{t}=0$, so that $\vec{P}=\epsilon_{0}\left(\epsilon_{s}-\epsilon_{\infty}\right) \vec{E}$ and $\vec{D}=\epsilon_{0} \epsilon_{s} \vec{E}$. For very high frequencies, $\tau \vec{P}_{t}$ dominates $\vec{P}$ so that $\vec{P} \approx 0$ and $\vec{D}=\epsilon_{0} \epsilon_{\infty} \vec{E}$ (thus the notation of $\infty$ ).

The Debye model is most often used to model electromagnetic wave interactions with water-based substances, such as biological materials. In particular, biological tissue is well represented by multi-pole Debye models, by accounting for permanent dipole moments in the water. The Debye model has other physical characteristics which make it attractive from an analytical point of view (for details, see [36]).

\subsubsection{Lorentz Model}

The Lorentz model for electronic polarization in differential form is represented with the second order equation:

$$
\frac{\partial^{2} \vec{P}}{\partial t^{2}}+\frac{1}{\tau} \frac{\partial \vec{P}}{\partial t}+\omega_{0}^{2} \vec{P}=\epsilon_{0} \epsilon_{d} \omega_{0}^{2} \vec{E},
$$

where $\epsilon_{d}=\epsilon_{s}-\epsilon_{\infty}$ and $\omega_{0}$ is the resonance frequency of the material.

The Lorentz model is formulated by modeling the atomic structure of the material as a damped vibrating system. Applying classical Newtonian laws of motion, the displacement of the outermost shell of the atom is found to be a solution to a second-order, ordinary differential equation [36].

\subsection{Reduction to One Dimension}

The electric field is assumed to be polarized to have oscillations in the $x$ - $z$ plane only, as described in [5]. Restricting the problem to one dimension, we can write the electric and magnetic fields, $\vec{E}$ and $\vec{H}$ respectively, as follows

$$
\begin{aligned}
\vec{E}(t, \vec{x}) & =\hat{i} E(t, z) \\
\vec{H}(t, \vec{x}) & =\hat{j} H(t, z),
\end{aligned}
$$

so that we are only concerned with the scalar values $E(t, z)$ and $H(t, z)$. In this case Maxwell's equations become:

$$
\begin{gathered}
\frac{\partial E}{\partial z}=-\mu_{0} \frac{\partial H}{\partial t} \\
-\frac{\partial H}{\partial z}=\frac{\partial D}{\partial t}+J_{s} .
\end{gathered}
$$

We take the partial derivative of Equation (2.7a) with respect to $z$, and the partial of Equation $(2.7 \mathrm{~b})$ with respect to $t$. Equating the $\frac{\partial^{2} H}{\partial z \partial t}$ terms in each, and thus eliminating the magnetic field $H$, we have:

$$
E^{\prime \prime}=\mu_{0}\left(\ddot{D}+\dot{J}_{s}\right)
$$


(where ${ }^{\prime}$ denotes $z$ derivatives and ${ }^{\circ}$ denotes time derivatives). Using the constitutive law for he electric flux density given by $D=\epsilon_{0} \epsilon_{\infty} E+P$, we have

$$
\mu_{0} \epsilon_{0} \epsilon_{\infty} \ddot{E}+\mu_{0} \ddot{P}-E^{\prime \prime}=-\mu_{0} \dot{J}_{s} \text { in } \Omega=[a, b] .
$$

In order to have a finite computational domain, we impose absorbing boundary conditions at $z=a$ and $z=b$, which are modeled as

$$
\left[\dot{E}-\mathrm{c} E^{\prime}\right]_{z=a}=0 ;\left[\dot{E}+\mathrm{c} E^{\prime}\right]_{z=b}=0,
$$

where $c=1 / \sqrt{\epsilon_{0} \mu_{0}}$ is the speed of light in vacuum. With these boundary conditions, any incident signal passes out of the computational domain, and does not return. The homogeneous initial conditions in 1D become

$$
E(0, z)=0, P(0, z)=0, \dot{P}(0, z)=0, \dot{E}(0, z)=0 .
$$

\section{Numerical Solution}

\subsection{Spatial Discretization Using Finite Elements}

We apply a finite element method using standard piecewise linear one dimensional basis elements to discretize the model (2.8) in space. Let $N$ be the number of intervals in the discretization of $z$, and $\Delta z=(b-a) / N$, then the finite element discretization has an order of accuracy of $O\left(\Delta z^{2}\right)$. The resulting system of ordinary differential equations after the spatial discretization is the semi-discrete form

$$
\epsilon_{\infty} \mu_{0} \epsilon_{0} M \ddot{e}+\mu_{0} M \ddot{p}+B \dot{e}+K e=\mu_{0} J
$$

with either

$$
\dot{p}+\lambda p=\epsilon_{0} \epsilon_{d} \lambda e,(\text { Debye Media) }
$$

or

$$
\ddot{p}+\lambda \dot{p}+\omega_{0}^{2} p=\epsilon_{0} \epsilon_{d} \omega_{0}^{2} e,(\text { Lorentz Media), }
$$

where $\lambda=\frac{1}{\tau}$. The vectors $e$ and $p$ represent the values of $E$, and $P$, respectively at the nodes $z_{i}=i \Delta z$. The mass matrix $M$ has entries

$$
M_{i j}=\left\langle\phi_{i}, \phi_{j}\right\rangle:=\int_{a}^{b} \phi_{i} \phi_{j} d z
$$

where $\left\{\phi_{i}\right\}_{i=0}^{N}$ are the basis functions. The stability matrix $K$ has entries

$$
K_{i j}=\left\langle\phi_{i}^{\prime}, \phi_{j}^{\prime}\right\rangle:=\int_{a}^{b} \phi_{i}^{\prime} \phi_{j}^{\prime} d z
$$

The matrix $B$ results from the boundary conditions where

$$
B_{i j}=\frac{1}{c}\left[\phi_{i}(a) \phi_{j}(a)-\phi_{i}(b) \phi_{j}(b)\right] .
$$


Finally, $J$ is defined as

$$
J_{i}=-\left\langle\phi_{i}, \dot{J}_{s}\right\rangle:=-\int_{a}^{b} \dot{J}_{s} \phi_{i} d z
$$

For Debye media, we differentiate (3.2) and substitute for $\ddot{p}$ into (3.1) to obtain an equation only dependent explicitly on $p$, given as

$$
\begin{aligned}
& \epsilon_{\infty} M \ddot{e}+\left(B+\epsilon_{d} \lambda M\right) \dot{e}+\left(c^{2} K-\epsilon_{d} \lambda^{2} M\right) e+\frac{\lambda^{2}}{\epsilon_{0}} M p=\frac{1}{\epsilon_{0}} J, \\
& \dot{p}+\lambda p-\epsilon_{0} \epsilon_{d} \lambda e=0 .
\end{aligned}
$$

In the above $c=1 / \sqrt{\epsilon_{0} \mu_{0}}$ is the speed of light in vacuum.

For Lorentz media we substitute (3.3) into (3.1) to get

$$
\begin{aligned}
& \epsilon_{\infty} M \ddot{e}+B \dot{e}+\left(c^{2} K+\epsilon_{d} \omega_{0}^{2} M\right) e-\frac{\omega_{0}^{2}}{\epsilon_{0}} M p-\frac{\lambda}{\epsilon_{0}} M \dot{p}=\frac{1}{\epsilon_{0}} J, \\
& \ddot{p}+\lambda \dot{p}+\omega_{0}^{2} p=\epsilon_{0} \epsilon_{d} \omega_{0}^{2} e .
\end{aligned}
$$

For linear finite elements in one dimension, the entries of the mass matrix $M$ are

$$
M_{i j}= \begin{cases}2 \Delta z / 3, & \text { if } 0<i=j<N \\ \Delta z / 3, & \text { else if } i=j=0 \text { or } N \\ \Delta z / 6, & \text { else if } i=j \pm 1\end{cases}
$$

The entries of the stiffness matrix can be calculated as

$$
K_{i j}= \begin{cases}2 / \Delta z, & \text { if } 0<i=j<N \\ 1 / \Delta z, & \text { else if } i=j=0 \text { or } N \\ -1 / \Delta z, & \text { else if } i=j \pm 1\end{cases}
$$

\subsection{Time Discretization Using Finite Differences}

In order to solve the semi-discrete form of our equations we may convert each coupled second order system of equations into one larger first order system and apply a theta method [22]. As our finite element method is second order in space, if we choose $\theta=\frac{1}{2}$ the discretization is second order in time as well (thus, we have used $\theta=\frac{1}{2}$ throughout). Therefore, for appropriately smooth data and with $\Delta t=O(\Delta z)$, the combined method is second order in time and space. For the system of equations corresponding to the Debye model we consider an additional method of temporal finite difference for comparison.

\subsubsection{Debye Media}

In the first method to be considered, we will convert the coupled second order system of equations into one larger first order system and simply apply a theta method as described above. In the second method, we will solve first for the polarization with a forward differencing scheme using the initial conditions, and then use polarization solution vector to update 
a second order central difference scheme for the magnitude of the electric field. We then continue this process iteratively, alternating between solving for $p$ and for $e$. Both methods are second order in time and space for appropriately smooth data (and with $\Delta t=O(h)$ ).

Method 1: For Debye media we convert (3.4a)-(3.4b) into a first order system of equations in three unknowns, $X=[e, p, d]^{T}$, where $d=\dot{e}$, resulting in

$$
\bar{M} \dot{X}+\bar{K} X=\bar{J},
$$

with

$$
\begin{gathered}
\bar{M}=\left[\begin{array}{ccc}
I & 0 & 0 \\
0 & I & 0 \\
0 & 0 & \epsilon_{\infty} M
\end{array}\right], \\
\bar{K}=\left[\begin{array}{ccc}
0 & 0 & -I \\
-\epsilon_{0} \epsilon_{d} \lambda & \lambda & 0 \\
\left(c^{2} K-\epsilon_{d} \lambda^{2} M\right) & \frac{\lambda^{2}}{\epsilon_{0}} M & B+\epsilon_{d} \lambda M
\end{array}\right],
\end{gathered}
$$

and

$$
\bar{J}=\left[\begin{array}{ccc}
0 & 0 & \frac{1}{\epsilon_{0}} J
\end{array}\right]^{T} .
$$

We apply a theta-scheme to (3.8) to obtain

$$
(\bar{M}+\theta \Delta t \bar{K}) X^{n+1}=(\bar{M}-(1-\theta) \Delta t \bar{K}) X^{n}+\left(\theta \bar{J}^{n+1}+(1-\theta) \bar{J}^{n}\right) .
$$

For $\theta=0.5$ the scheme can be written as

$$
\left(\bar{M}+\frac{\Delta t}{2} \bar{K}\right) X^{n+1}=\left(\bar{M}-\frac{\Delta t}{2} \bar{K}\right) X^{n}+\frac{1}{2}\left(\bar{J}^{n+1}+\bar{J}^{n}\right) .
$$

where, $\bar{J}^{n}=\bar{J}(n \Delta t)$ and $X^{n}=X(n \Delta t)$.

As we are assuming a fixed time step $\Delta t$, the matrix to be inverted does not change over time. Therefore, we solve for the LU factorization at the beginning and use back substitution at each time step. Since the matrices $\bar{M}$ and $\bar{K}$ are sparse, we employ the sparse matrix package UMFPACK [13]. For problems where the number of time steps is small compared with the number of nodes, it may be more efficient to use an iterative solver with the previous solution vector as the initial iterate.

Method 2: In our second method we use a second order central difference scheme to solve (3.4a). Our approach is to first solve for $p$ using a $\theta$-method, and then use that approximation to solve for $e$ at the next time step. Thus, our finite difference approximation for $(3.4 \mathrm{~b})$ is

$$
p^{n+1}=p^{n}-\Delta t \lambda p^{n+\theta}+\Delta t \lambda \epsilon_{d} e^{n+\theta}
$$

where $v^{n+\theta}=\theta v^{n+1}+(1-\theta) v^{n}$, for $v=e$ or $v=p$. This implies

$$
p^{n+1}=p^{n}+\frac{\lambda \Delta t}{1+\lambda \Delta t \theta}\left(\epsilon_{d} e^{n+\theta}-p_{n}\right) .
$$


Once we have $p^{n+1}$ we can solve for $e^{n+2}$. Applying second order central difference with averaging to $(3.4 \mathrm{a})$ gives

$$
\begin{aligned}
& \frac{1}{\Delta t^{2}} \epsilon_{\infty} M\left(e^{n+2}-2 e^{n+1}+e^{n}\right)+\frac{1}{2 \Delta t}\left(B+\epsilon_{d} \lambda M\right)\left(e^{n+2}-e^{n}\right) \\
& +\frac{1}{4}\left(c^{2} K-\epsilon_{d} \lambda^{2} M\right)\left(e^{n+2}+2 e^{n+1}+e^{n}\right)=\frac{1}{\epsilon_{0}} J^{n+1}-\frac{\lambda^{2}}{\epsilon_{0}} M \bar{p}^{n+1} .
\end{aligned}
$$

Defining $h_{\tau}=\lambda \Delta t$ and solving for the $e^{n+2}$ term we have

$$
\begin{aligned}
& {\left[\left(\epsilon_{\infty}+\frac{\epsilon_{d} h_{\tau}}{2}-\frac{\epsilon_{d} h_{\tau}^{2}}{4}\right) M+\frac{1}{2} \Delta t B\right] e^{n+2}=\left[\left(2 \epsilon_{\infty}+\frac{\epsilon_{d} h_{\tau}^{2}}{2}\right) M-\frac{c^{2} \Delta t^{2}}{2} K\right] e^{n+1}} \\
& -\left[\left(\epsilon_{\infty}-\frac{\epsilon_{d} h_{\tau}}{2}-\frac{\epsilon_{d} h_{\tau}^{2}}{4}\right) M+\frac{c^{2} \Delta t^{2}}{4} K+\frac{1}{2} \Delta t B\right] e^{n}+\frac{\Delta t^{2}}{\epsilon_{0}} J^{n+1}-\frac{h_{\tau}^{2}}{\epsilon_{0}} M p^{n+1}
\end{aligned}
$$

or equivalently,

$$
A_{1} e^{n+2}=A_{2} e^{n+1}+A_{3} e^{n}+\frac{\Delta t^{2}}{\epsilon_{0}} J^{n+1}-\frac{h_{\tau}^{2}}{\epsilon_{0}} M p^{n+1} .
$$

Note that in this case $A_{1}$ is tridiagonal and the matrix is the same for each time step, so we may store the Crout LU factorization and use back substitution to solve the system at each time step. For tridiagonal matrices the factorization and the back substitution are both order $O(N)[22]$.

Again, for $\theta=\frac{1}{2},(3.15)$ will be second order in time if the corresponding solution is $C^{3}$ in time. Equation (3.18) is also second order in time assuming an exact solution for $P$, and that $E$ has four continuous time derivatives (for the second order difference approximation). The truncation error for this approximation is

$$
T\left(t_{n}\right)=\Delta t^{2}\left(\frac{1}{12} E^{(4)}+\frac{1}{6} E^{(3)}+\frac{1}{4} E^{(2)}\right) .
$$

Therefore, since the semi-discrete form is $O\left(h^{2}\right)$, this approximation method overall is $O\left(h^{2}\right)$ when $\Delta t=O(h)$.

\subsubsection{Lorentz Media}

For Lorentz media we convert (3.5a)-(3.5b) into a first order system of equations in three unknowns, $X=[e, p, d, q]^{T}$, where $d=\dot{e}, q=\dot{p}$, resulting in

$$
\bar{M} \dot{X}+\bar{K} X=\bar{J}
$$

with

$$
\bar{M}=\left[\begin{array}{cccc}
I & 0 & 0 & 0 \\
0 & I & 0 & 0 \\
0 & 0 & \epsilon_{\infty} M & 0 \\
0 & 0 & 0 & I
\end{array}\right],
$$




$$
\bar{K}=\left[\begin{array}{cccc}
0 & 0 & -I & 0 \\
0 & 0 & 0 & -I \\
\left(c^{2} K+\epsilon_{d} \omega_{0}^{2} M\right) & -\frac{\omega_{0}^{2}}{\epsilon_{0}} M & B & -\frac{\lambda}{\epsilon_{0}} M \\
-\epsilon_{0} \epsilon_{d} \omega_{0}^{2} & \omega_{0}^{2} & 0 & \lambda
\end{array}\right]
$$

and

$$
\bar{J}=\left[\begin{array}{llll}
0 & 0 & \frac{1}{\epsilon_{0}} J & 0
\end{array}\right]^{T} .
$$

As before, we apply a theta-scheme to $\bar{M} \dot{X}+\bar{K} X=\bar{J}$ to obtain

$$
(\bar{M}+\theta \Delta t \bar{K}) X^{n+1}=(\bar{M}-(1-\theta) \Delta t \bar{K}) X^{n}+\left(\theta \bar{J}^{n+1}+(1-\theta) \bar{J}^{n}\right),
$$

For $\theta=0.5$ the scheme can be written as

$$
\left(\bar{M}+\frac{\Delta t}{2} \bar{K}\right) X^{n+1}=\left(\bar{M}-\frac{\Delta t}{2} \bar{K}\right) X^{n}+\frac{1}{2}\left(\bar{J}^{n+1}+\bar{J}^{n}\right) .
$$

where, $\bar{J}^{n}=\bar{J}(n \Delta t)$ and $X^{n}=X(n \Delta t)$.

Again we are assuming a fixed $\Delta t$, therefore we solve for the LU factorization at the beginning and use back substitution at each time step.

\section{Stability Analysis}

Fourier analysis is an important tool in the study of stability of finite difference and finite element schemes. The Fourier transform of a function gives an alternative representation of the function, and one can infer certain properties of a function from its Fourier transform. The Fourier inversion formula represents a function as a superposition of waves $\mathrm{e}^{i \omega z}$ with different amplitudes that are given by the Fourier transform. Under the Fourier transform the operation of differentiation is converted into the operation of multiplication by $\mathrm{i} \omega$.

An important application of Fourier analysis is the von Neumann analysis of stability of difference schemes. With the use of Fourier analysis we can give necessary and sufficient conditions for the stability of these schemes. For a difference scheme, advancing the solution of the scheme by one time step is equivalent to multiplying the Fourier transform of the solution by an amplification factor. The amplification factor is so called because its magnitude is the amount that the amplitude of each frequency in the solution, given by the Fourier transform of the solution at time step $n$, is amplified in advancing the solution to time step $n+1$. All the information of a scheme is contained in its amplification factor. In particular, the stability and accuracy of schemes can be determined from the amplification factor. Using Fourier analysis on the scheme to calculate the amplification factor $\zeta$ is equivalent to relacing discrete values of any unknown field vector $v_{m}^{n}$, at time $t=n \Delta t$ and spatial position $z=m \Delta z$, in the scheme by $\zeta^{n} e^{\mathrm{i} m \theta}$ for each value of $n$ and $m$. The resulting equation is a polynomial in $\zeta$ and is called the stability polynomial or the characteristic polynomial for the scheme. The stability polynomial usually depends on the discretization parameters, such as the time step and the mesh step size as well as the medium parameters. The roots of this polynomial can be obtained and will determine the stability of the scheme. 
Since the roots of the polynomial are the amplification factors of the scheme, the scheme will be stable if the amplitude of the roots is less than or equal to 1 .

We follow [28] to derive stability results for the finite element schemes presented here. Since the boundary conditions do not affect the stability and dispersion properties of the scheme in the interior of the domain, we neglect the effects of boundary conditions $(B=0$ in (3.1)) in our analysis. As the stability and dispersion properties of the scheme are also independent of the source $J_{s}$, we take $J=0$ in (3.1) without loss of generality.

\subsection{Free Space}

For the stability analysis in free space we have $P=0$. Thus, in equation (3.1) we also substitute $\ddot{P}=0$. In free space we have the equation

$$
\mu_{0} \epsilon_{0} M \ddot{e}+K e=0 .
$$

In the above we have also assumed that $\epsilon_{\infty}=1$. Using $\mathrm{c}=1 / \sqrt{\epsilon_{0} \mu_{0}}$, and rewriting (4.1) in first order form in the variables $X=[e, d]^{T}$, where $d=\dot{e}$, we have

$$
\left[\begin{array}{cc}
I & 0 \\
0 & \frac{1}{c^{2}} M
\end{array}\right] \dot{X}+\left[\begin{array}{cc}
0 & -I \\
K & 0
\end{array}\right] X=0 .
$$

Applying a theta scheme to (4.2) we get

$$
\left[\begin{array}{cc}
I & -\theta \Delta t I \\
\theta \Delta t K & \frac{1}{c^{2}} M
\end{array}\right] X^{n+1}=\left[\begin{array}{cc}
I & (1-\theta) \Delta t I \\
-(1-\theta) \Delta t K & \frac{1}{c^{2}} M
\end{array}\right] X^{n}=0 .
$$

We note the following identities associated with the application of the mass and the stiffness matrices on vectors $\phi_{j}^{n}=\tilde{\phi} \zeta^{n} \mathrm{e}^{i(k j \Delta z)}$.

1. For the mass matrix $M$ we have

$$
\begin{aligned}
M \phi_{j}^{n} & =\frac{\Delta z}{6} \phi_{j-1}^{n}+\frac{2 \Delta z}{3} \phi_{j}^{n}+\frac{\Delta z}{6} \phi_{j+1}^{n} \\
& =\left(\mathrm{e}^{-i k \Delta z}+4+\mathrm{e}^{i k \Delta z}\right) \frac{\Delta z}{6} \phi_{j}^{n} \\
& =\left(3-2 \sin ^{2}(k \Delta z / 2)\right) \frac{\Delta z}{3} \phi_{j}^{n}
\end{aligned}
$$

2. For the stiffness matrix $K$ we have

$$
\begin{aligned}
K \phi_{j}^{n} & =-\frac{1}{\Delta z} \phi_{j-1}^{n}+\frac{2}{\Delta z} \phi_{j}^{n}-\frac{1}{\Delta z} \phi_{j+1}^{n} \\
& =\left(\mathrm{e}^{-i k \Delta z}-2+\mathrm{e}^{i k \Delta z}\right) \frac{-1}{\Delta z} \phi_{j}^{n} \\
& \left.=\frac{4}{\Delta z} \sin ^{2}(k \Delta z / 2)\right) \phi_{j}^{n} .
\end{aligned}
$$


We also define two quantities

$$
\begin{aligned}
& \kappa=3-2 \sin ^{2}\left(\frac{k \Delta z}{2}\right), \\
& \eta=3 \nu^{2} \sin ^{2}\left(\frac{k \Delta z}{2}\right),
\end{aligned}
$$

where the Courant number is

$$
\nu=\frac{\mathrm{c} \Delta t}{\Delta z},\left(\epsilon_{\infty}=1\right)
$$

In a general dispersive material $\nu=\frac{\mathrm{c} \Delta t}{\sqrt{\epsilon_{\infty}} \Delta z}$. In air $\epsilon_{\infty}=1$. To determine the stability criterion for the finite element scheme in free space we now substitute

$$
X_{j}^{n}=\left[\begin{array}{c}
e_{j}^{n} \\
d_{j}^{n}
\end{array}\right]=\left[\begin{array}{c}
\tilde{e} \\
\tilde{d}
\end{array}\right] \zeta^{n} \mathrm{e}^{\mathrm{i}(k j \Delta z)},
$$

into the discrete equations (4.3) with $\theta=1 / 2$. Here $k$ is the wave number, Using the identities (4.4) and (4.5) we obtain a homogeneous linear system of the form

$$
\mathcal{A} \tilde{\mathcal{X}}=0
$$

with

$$
\mathcal{A}=\left[\begin{array}{cc}
\zeta-1 & -\frac{\Delta t}{2}(\zeta+1) \\
\frac{2 \Delta t}{\Delta z} \sin ^{2}\left(\frac{k \Delta z}{2}\right)(\zeta+1) & \frac{\Delta z}{3 c^{2}}\left(3-2 \sin ^{2}\left(\frac{k \Delta z}{2}\right)\right)(\zeta-1)
\end{array}\right]
$$

and

$$
\tilde{\mathcal{X}}=[\tilde{e}, \tilde{d}]^{T}
$$

By setting the determinant of $\mathcal{A}$ to be zero, we obtain the stability polynomial

$$
\zeta^{2}-2 \zeta\left(\frac{\kappa^{2}-\eta^{2}}{\kappa^{2}+\eta^{2}}\right)+1=0,
$$

From (4.12) we can show that $|\zeta|=1$ always, regardless of the medium parameters. This implies that the finite element scheme with the theta method $(\theta=1 / 2)$ in free space is unconditionally stable as well as non-dissipative.

\subsection{Debye Media}

To determine the stability conditions for the finite element scheme described as Method 1 in Section 3.2.1 for Debye media we substitute

$$
X_{j}^{n}=\left[\begin{array}{c}
e_{j}^{n} \\
p_{j}^{n} \\
d_{j}^{n}
\end{array}\right]=\left[\begin{array}{c}
\tilde{e} \\
\tilde{p} \\
\tilde{d}
\end{array}\right] \zeta^{n} \mathrm{e}^{\mathrm{i} k j \Delta z},
$$


in the discrete equations (3.13) (with $J=0$ ). As in the case of free space, we obtain a homogeneous system of the type $\mathcal{A} \tilde{\mathcal{X}}=0$. We then set $\operatorname{det}(\mathcal{A})=0$ to obtain the stability polynomial

$$
a_{3} \zeta^{3}+a_{2} \zeta^{2}+a_{1} \zeta+a_{0}=0
$$

where the coefficients of the stability polynomial are given as

$$
\begin{aligned}
& a_{3}=\eta^{2}\left(h_{\tau}+2\right)+\kappa^{2}\left(h_{\tau} \epsilon_{s}+2 \epsilon_{\infty}\right), \\
& a_{2}=\eta^{2}\left(3 h_{\tau}+2\right)-\kappa^{2}\left(h_{\tau} \epsilon_{s}+6 \epsilon_{\infty}\right), \\
& a_{1}=\eta^{2}\left(3 h_{\tau}-2\right)-\kappa^{2}\left(h_{\tau} \epsilon_{s}-6 \epsilon_{\infty}\right), \\
& a_{0}=\eta^{2}\left(h_{\tau}-2\right)+\kappa^{2}\left(h_{\tau} \epsilon_{s}-2 \epsilon_{\infty}\right),
\end{aligned}
$$

where $\eta$, and $\kappa$ are as defined in (4.6b), and (4.6a), respectively, and $h_{\tau}=\Delta t / \tau$.

To determine the stability polynomial for the finite element scheme described in Method 2 in Section (3.2.1), we substitute (4.13) in the discrete equations (3.17). Following the procedure discussed above we obtain the stability polynomial

$$
b_{3} \zeta^{3}+b_{2} \zeta^{2}+b_{1} \zeta+b_{0}=0
$$

with coefficients

$$
\begin{aligned}
& b_{3}=\eta^{2}\left(h_{\tau}+2\right)+\kappa^{2}\left(h_{\tau} \epsilon_{s}+2 \epsilon_{\infty}\right)-\frac{\kappa^{2} h_{\tau}^{3} \epsilon_{d}}{4}, \\
& b_{2}=\eta^{2}\left(3 h_{\tau}+2\right)-\kappa^{2}\left(h_{\tau} \epsilon_{s}+6 \epsilon_{\infty}\right)+\frac{\kappa^{2} h_{\tau}^{3} \epsilon_{d}}{4}, \\
& b_{1}=\eta^{2}\left(3 h_{\tau}-2\right)-\kappa^{2}\left(h_{\tau} \epsilon_{s}-6 \epsilon_{\infty}\right)+\frac{\kappa^{2} h_{\tau}^{3} \epsilon_{d}}{4}, \\
& b_{0}=\eta^{2}\left(h_{\tau}-2\right)+\kappa^{2}\left(h_{\tau} \epsilon_{s}-2 \epsilon_{\infty}\right)-\frac{\kappa^{2} h_{\tau}^{3} \epsilon_{d}}{4},
\end{aligned}
$$

If we neglect terms involving $h_{\tau}^{3}$, the stability polynomial of Method 2 for Debye is the same as in Method 1. Thus for small values of $h_{\tau}$, the two methods have the same stability properties. In the rest of this section we refer to Method 1 as the finite element scheme and we will compare stability properties of this scheme with those of the finite difference scheme [18] analyzed in [28].

In Figure 1 we plot the absolute value of the largest root of (4.14) for the finite element scheme with $\nu=1$ as a function of $k \Delta z$. In Figure 2 we plot the the absolute value of the largest root of the stability polynomial of the finite difference scheme JHT [18], with $\nu=1$ as a function of $k \Delta z$.

In Figure 3 (left) we plot the absolute value of the largest root of (4.14) for the finite element scheme with $\nu=1$ as a function of the wave number $k$. In Figure 3 (right) we plot the the absolute value of the largest root of the stability polynomial of the finite difference scheme JHT [18], with $\nu=1$ as a function of $k$.

To generate these plots we assumed the following values of the physical parameters: $\tau=8.1 \times 10^{-12}, \epsilon_{\infty}=1$, and $\epsilon_{s}=78.2$. The time step $\Delta t$ is determined by the choice of $h_{\tau}$ and the physical parameter $\tau$. These plots show varying values of $h_{\tau}$ from 0.1 to 0.001 . 

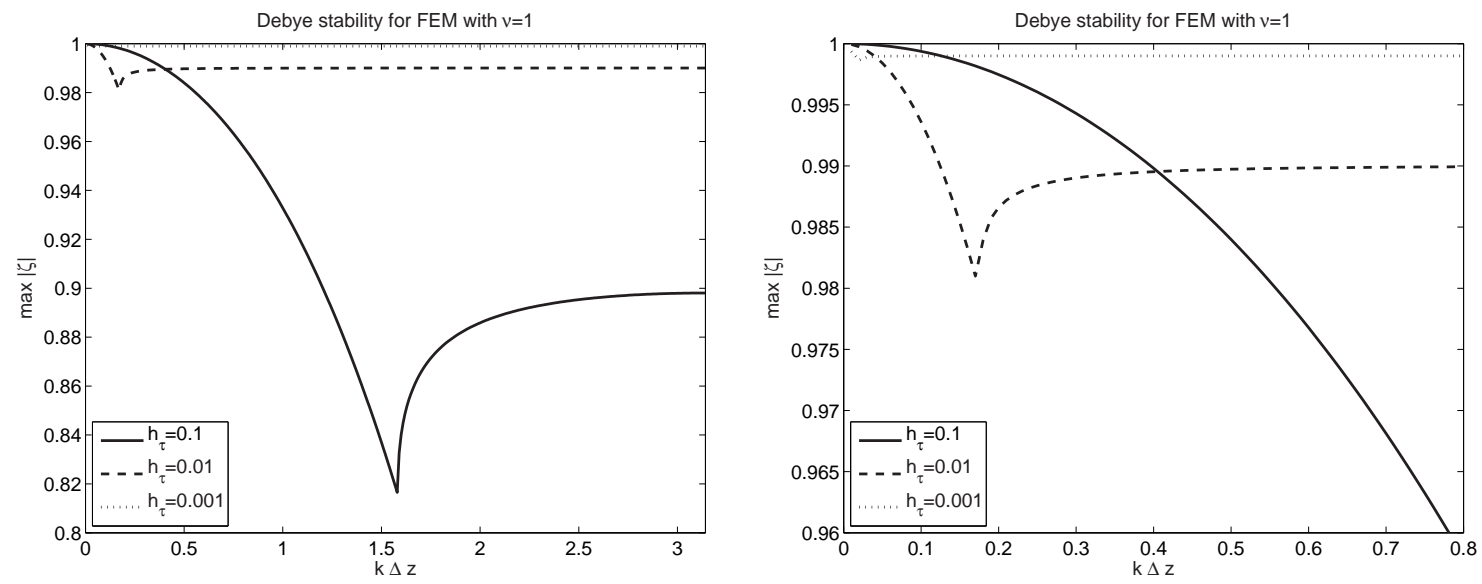

Figure 1: Plot of $\max |\zeta|$ versus $k \Delta z$ for $h_{\tau}=\{0.1,0.01,0.001\}$ for the finite element scheme in a Debye medium, with $\nu=1$. (Right plot is zoom of left plot.)

From the plots we can see that the dissipation of the numerical schemes can be reduced by decreasing $h_{\tau}$. For stability and least dissipation, $h_{\tau}=0.001$ is recommended. For the finite element scheme increasing $\nu$ from 1 to 16 does not seem to change the stability behaviour of the scheme. This suggests the unconditional stability of the finite element scheme. However, the finite difference scheme is conditionally stable and has the stability criteria $\nu \leq 1$. The stability criteria for the finite difference scheme have been derived in $[28,10]$.

In Figure 4 we plot $\max |\zeta|$ versus $k \Delta z$ for $h_{\tau}=0.1$ (left) and $h_{\tau}=0.3$ for the finite element schemes of Method 1 (FEM1) and Method 2 (FEM2) in a Debye medium, with $\nu=1$. From this plot we can see a slight difference in the two schemes when $h_{\tau}=0.3$. However, for $h_{\tau}=0.1$ the two schemes produce the same plots. 

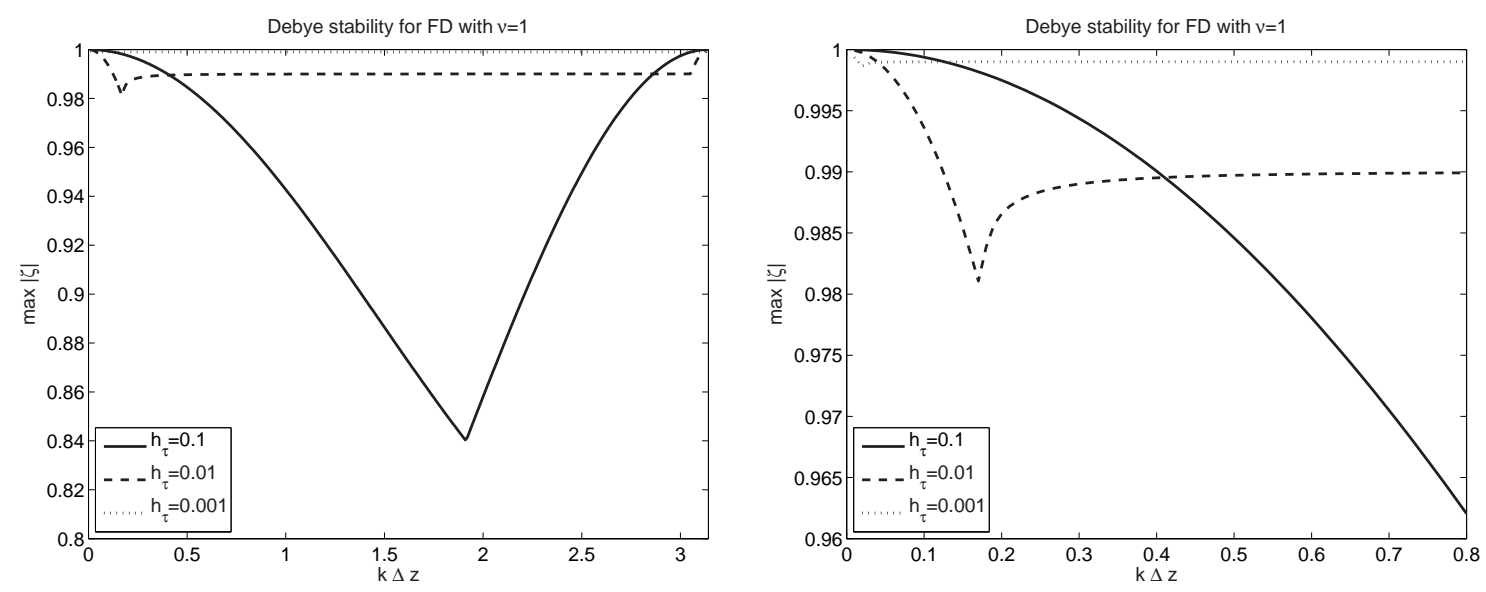

Figure 2: Plot of $\max |\zeta|$ versus $k \Delta z$ for $h_{\tau}=\{0.1,0.01,0.001\}$ for the finite difference scheme JHT in a Debye medium, with $\nu=1$. (Right plot is zoom of left plot.)
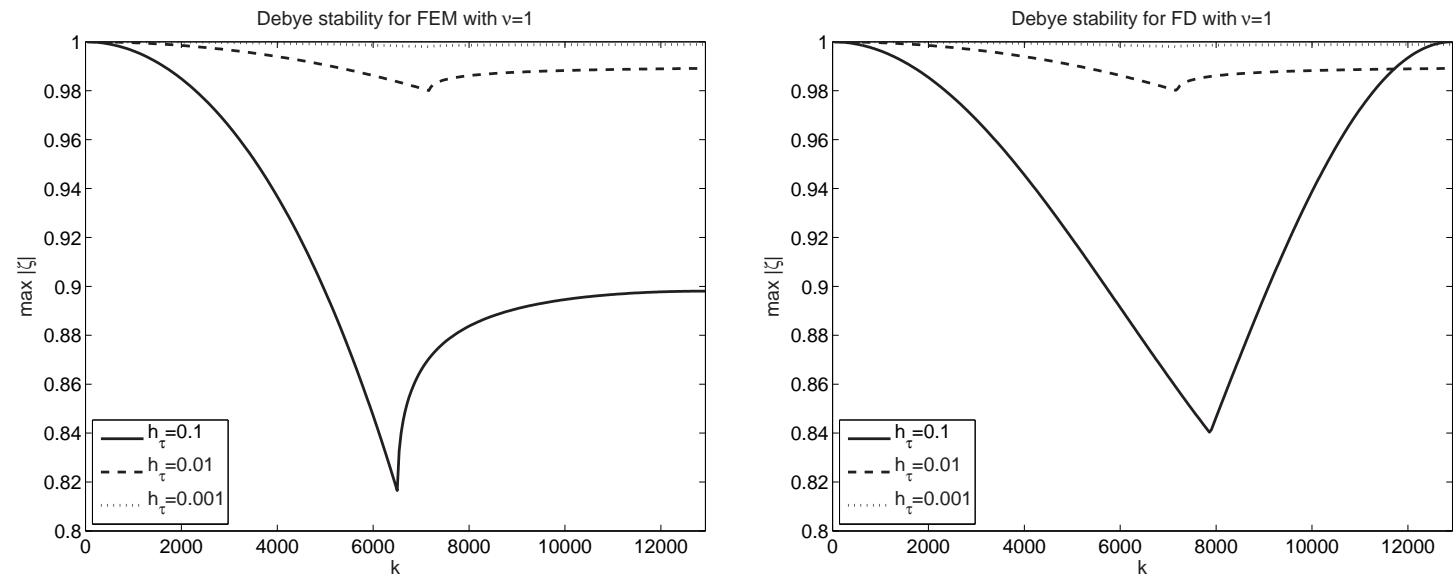

Figure 3: Plot of $\max |\zeta|$ versus the wave number $k$ for $h_{\tau}=\{0.1,0.01,0.001\}$ for the finite element scheme (left) and the finite difference scheme (right) in a Debye medium, with $\nu=1$. 

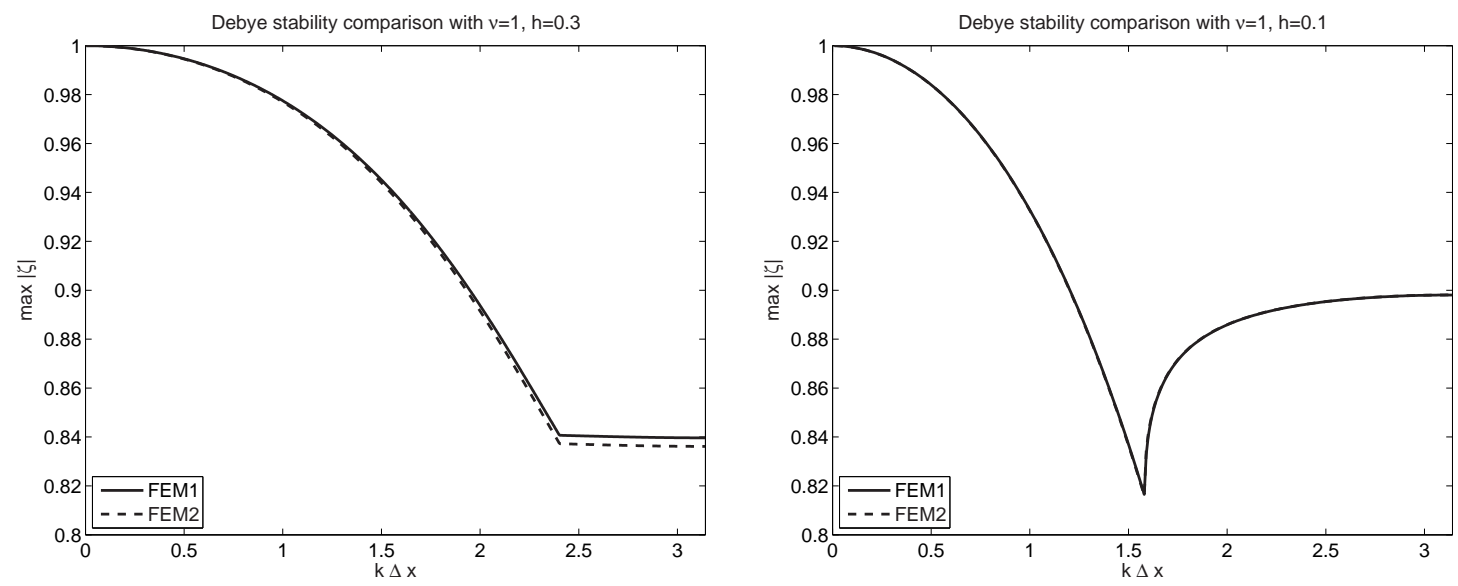

Figure 4: Plots of $\max |\zeta|$ versus $k \Delta z$ for $h_{\tau}=0.1$ (left) and $h_{\tau}=0.3$ for the finite element schemes of Method 1 (FEM1) and Method 2 (FEM2) in a Debye medium, with $\nu=1$.

\subsection{Lorentz Media}

To determine the stability conditions for Lorentz media we substitute

$$
X_{j}^{n}=\left[\begin{array}{c}
e_{j}^{n} \\
p_{j}^{n} \\
d_{j}^{n} \\
q_{j}^{n}
\end{array}\right]=\left[\begin{array}{c}
\tilde{e} \\
\tilde{p} \\
\tilde{d} \\
\tilde{q}
\end{array}\right] \zeta^{n} \mathrm{e}^{\mathrm{i} k j \Delta z}
$$

in the discrete equations (3.24). As in the case of free space, we obtain a homogeneous system of the type $\mathcal{A} \tilde{\mathcal{X}}=0$. We then $\operatorname{set} \operatorname{det}(\mathcal{A})=0$ to obtain the stability polynomial

$$
a_{4} \zeta^{4}+a_{3} \zeta^{3}+a_{2} \zeta^{2}+a_{1} \zeta+a_{0}=0
$$

where the coefficients of the stability polynomial are given as

$$
\begin{aligned}
& a_{4}=\eta^{2}\left(2 \pi^{2} h_{0}^{2}+h_{\tau}+2\right)+\kappa^{2}\left(2 \pi^{2} h_{0}^{2} \epsilon_{s}+h_{\tau} \epsilon_{\infty}+2 \epsilon_{\infty}\right) \\
& a_{3}=\eta^{2}\left(8 \pi^{2} h_{0}^{2}+2 h_{\tau}\right)-\kappa^{2} \epsilon_{\infty}\left(8+2 h_{\tau}\right) \\
& a_{2}=\eta^{2}\left(12 \pi^{2} h_{0}^{2}-4\right)-\kappa^{2}\left(4 \pi^{2} h_{0}^{2} \epsilon_{s}-12 \epsilon_{\infty}\right) \\
& a_{1}=\eta^{2}\left(8 \pi^{2} h_{0}^{2}-2 h_{\tau}\right)-\kappa^{2} \epsilon_{\infty}\left(8-2 h_{\tau}\right) \\
& a_{0}=\eta^{2}\left(2 \pi^{2} h_{0}^{2}-h_{\tau}+2\right)-\kappa^{2}\left(2 \pi^{2} h_{0}^{2} \epsilon_{s}-h_{\tau} \epsilon_{\infty}+2 \epsilon_{\infty}\right),
\end{aligned}
$$

where $\eta$, and $\kappa$ are as defined in (4.6b), and (4.6a), respectively, $h_{\tau}=\Delta t / \tau$, and $h_{0}=\Delta t / T_{0}$, where $T_{0}=2 \pi / \omega_{0}$.

We plot the absolute value of the largest root of (4.25) for $\nu=1$ versus $k \Delta z$ for the finite element scheme in Figures 5, and for the finite difference schemes JHT [18] and KF [19] in Figures 6 and 7. 

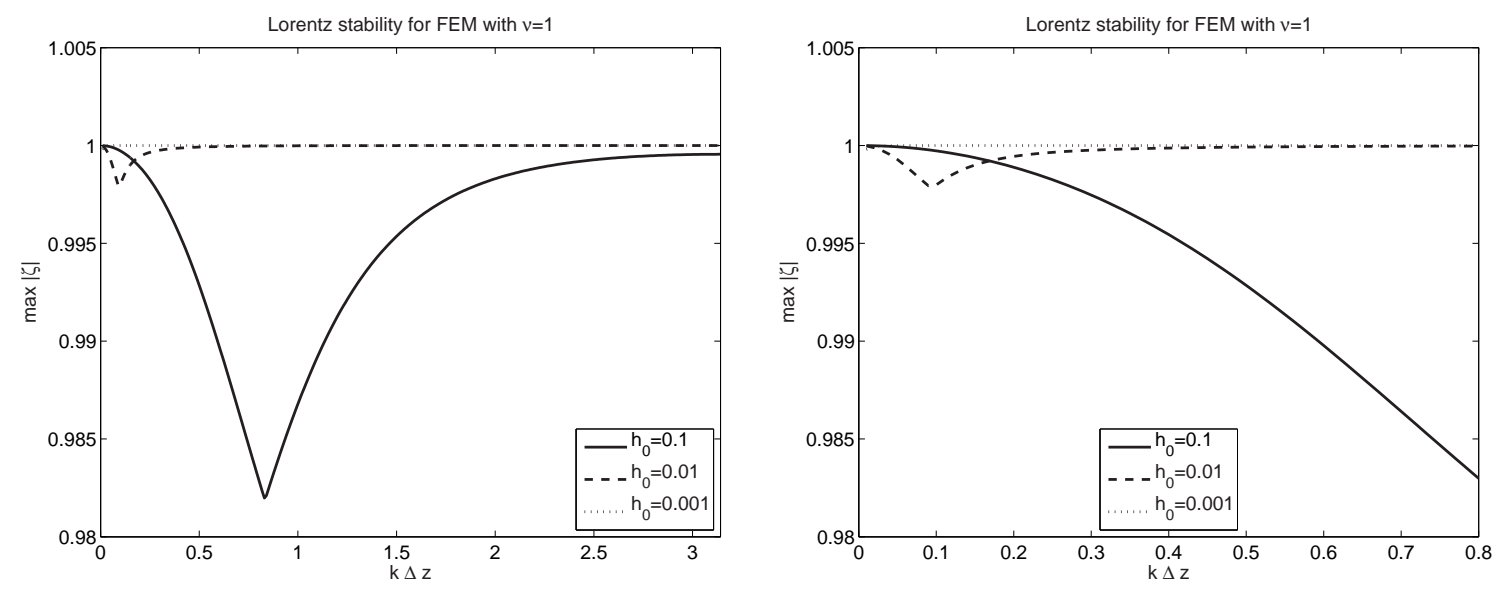

Figure 5: Plot of $\max |\zeta|$ versus $k \Delta z$ for $h_{\tau}=\{0.1,0.01,0.001\}$ for the finite element scheme in a Lorentz medium, with $\nu=1$. (Right plot is zoom of left plot.)

We then plot the absolute value of the largest root of (4.25) for $\nu=1$ versus the wave number $k$ for the finite element scheme in Figures 8, and for the finite difference schemes JHT and KF in Figures 9.

To generate these plots we assumed the following values for physical parameters: $\tau=$ $1.786 \times 10^{-16}, \epsilon_{\infty}=1, \epsilon_{s}=2.25$, and $\omega_{0}=4 \times 10^{16}$. For the Lorentz medium, all time scales must be properly resolved, therefore the time step $\Delta t$ is determined by the choice of either $h_{\tau}$ or $h_{0}$, whichever is most restrictive. For the current parameter values, $T_{0}<\tau$, thus $h_{0}$ is used. The plots show varying values of $h_{0}$ from 0.1 to 0.001 . From the plots we can see that the dissipation of the numerical schemes can be reduced by decreasing $h_{0}$. For stability and least dissipation, $h_{0}=0.01$ is recommended.

As in the case of the finite element method for Debye media, we see that increasing $\nu$ from 1 to 16 does not affect the stability properties of the finite element scheme for Lorentz media. This suggests the unconditional stability of the finite element method. However the finite difference scheme is again conditionally stable with the criteria $\nu \leq 1$. The stability criteria for the finite difference scheme for Lorentz media have also been derived in $[28,10]$. 

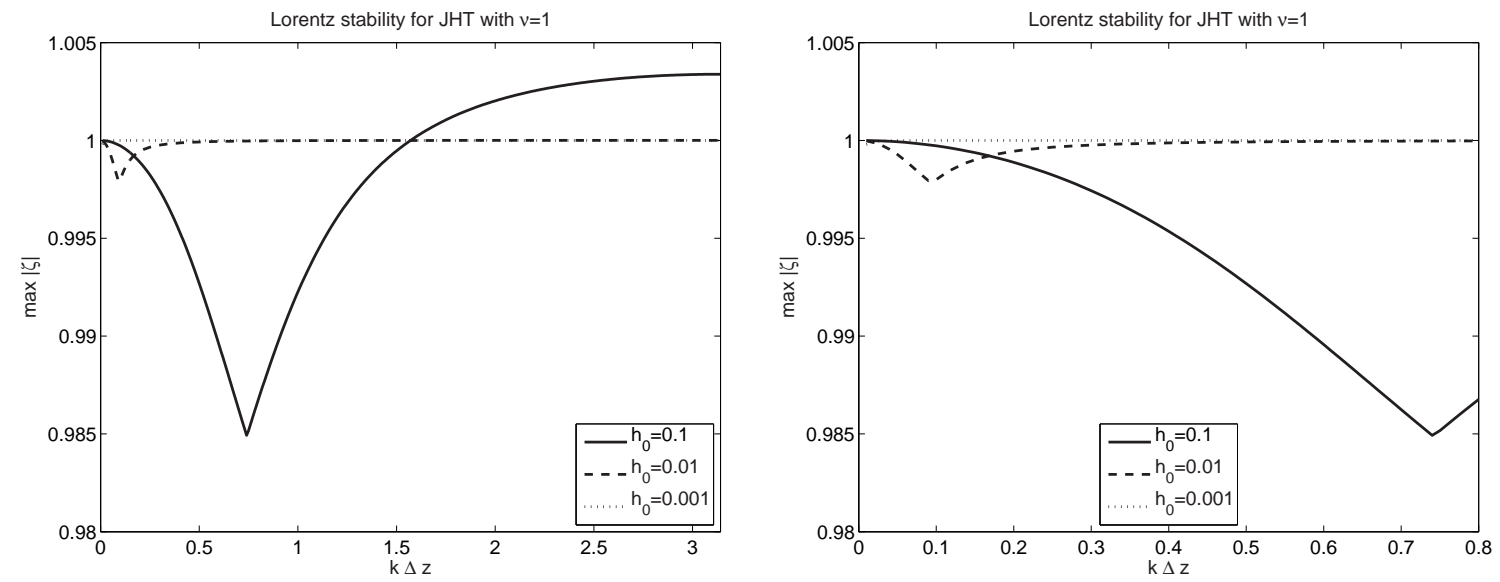

Figure 6: Plot of $\max |\zeta|$ versus $k \Delta z$ for $h_{\tau}=\{0.1,0.01,0.001\}$ for the JHT finite difference scheme in a Lorentz medium, with $\nu=1$. (Right plot is zoom of left plot.)
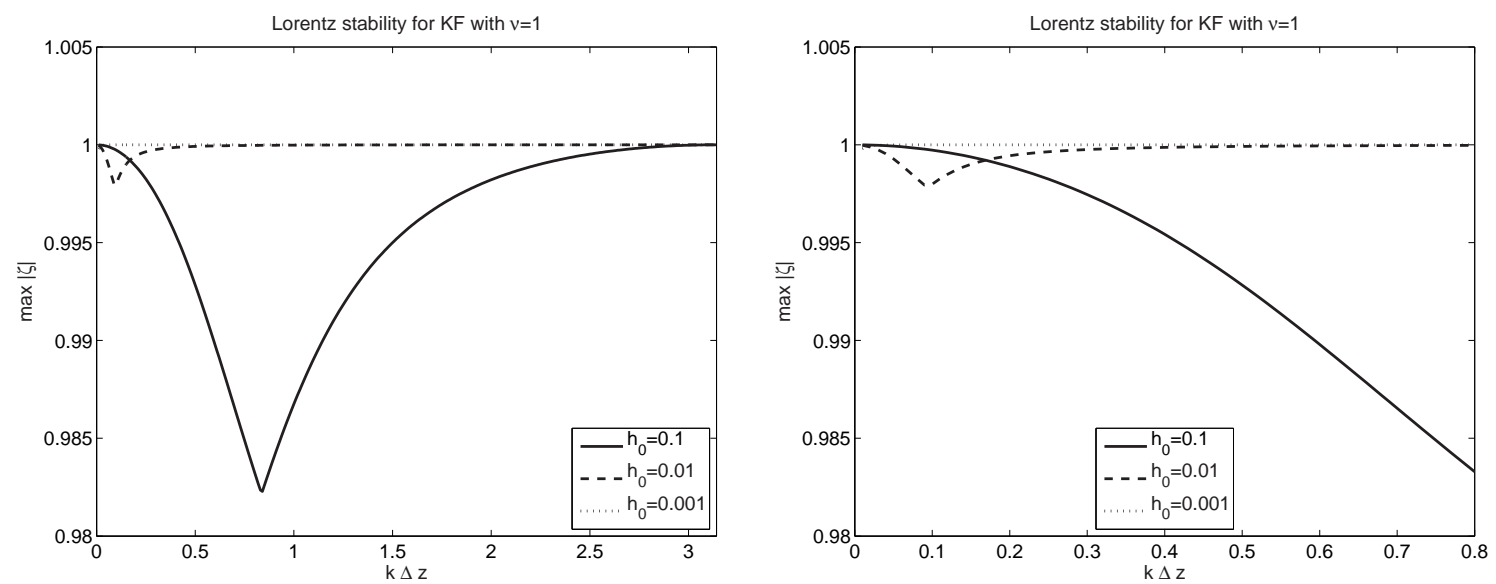

Figure 7: Plot of $\max |\zeta|$ versus $k \Delta z$ for $h_{\tau}=\{0.1,0.01,0.001\}$ for the KF finite difference scheme in a Lorentz medium, with $\nu=1$. (Right plot is zoom of left plot.) 


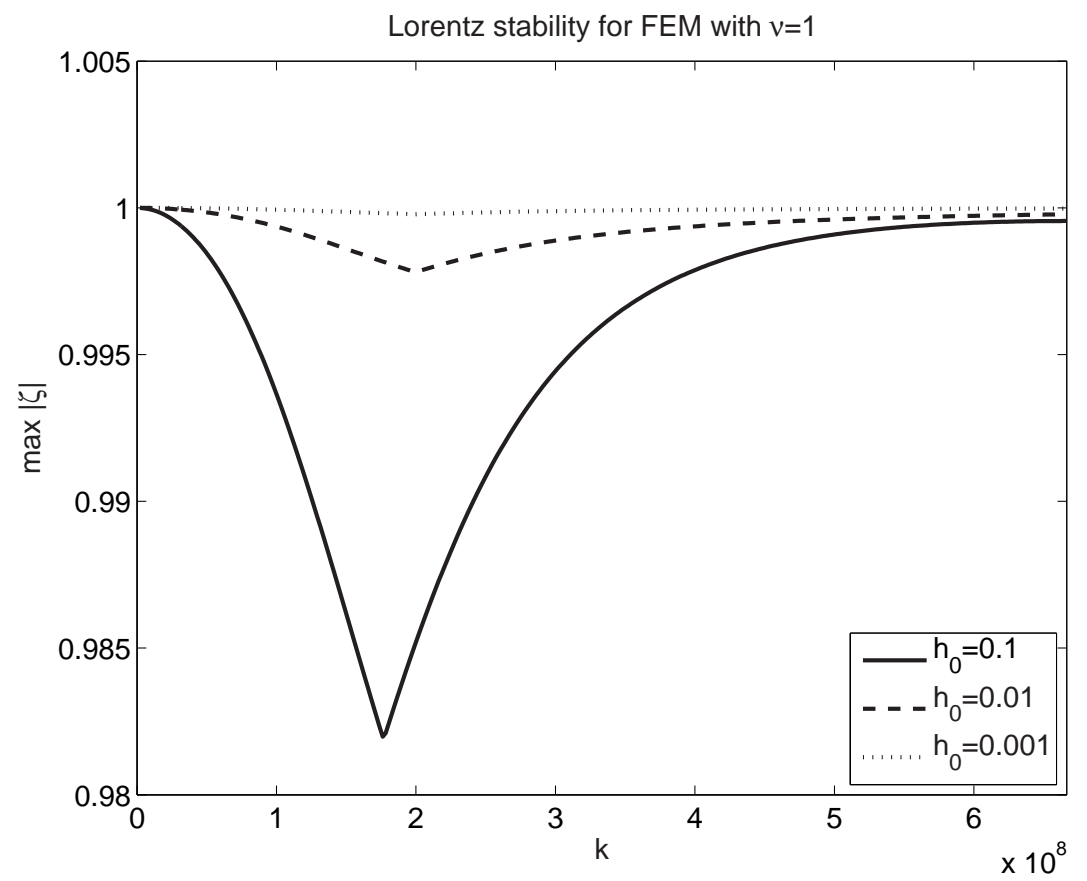

Figure 8: Plot of $\max |\zeta|$ versus the wave number $k$ for $h_{\tau}=\{0.1,0.01,0.001\}$ for the KF finite element scheme in a Lorentz medium, with $\nu=1$.
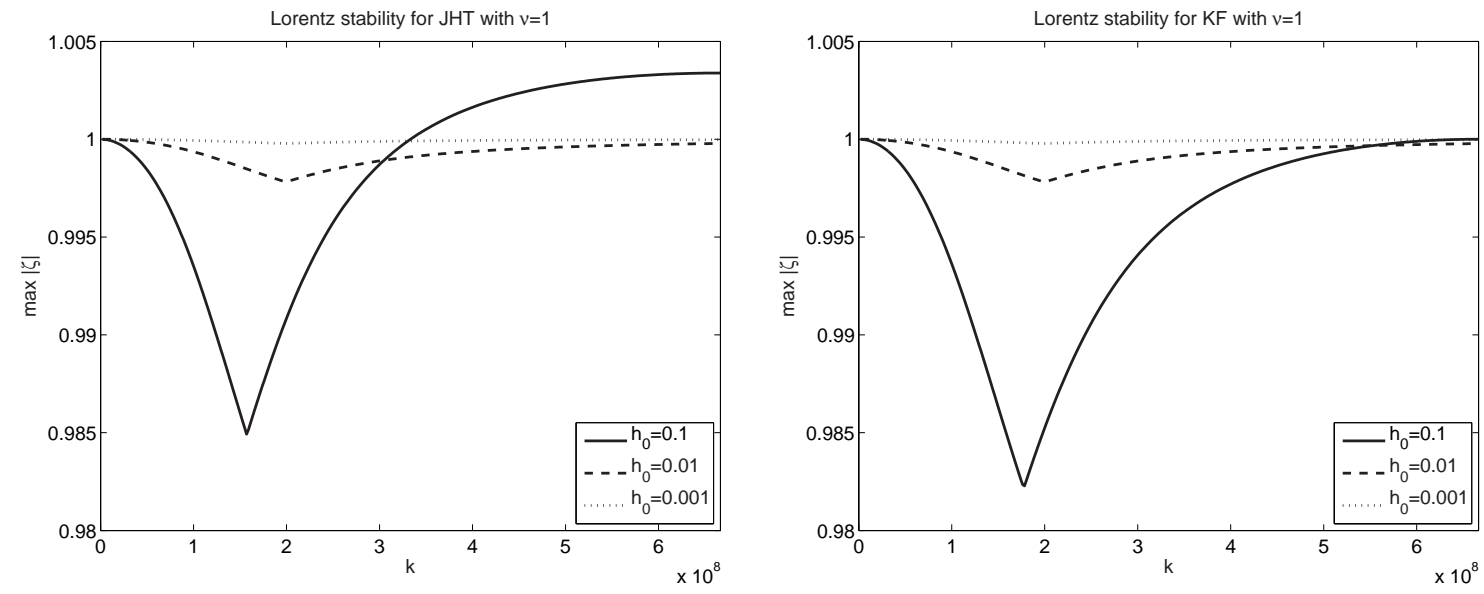

Figure 9: Plot of $\max |\zeta|$ versus the wave number $k$ for $h_{\tau}=\{0.1,0.01,0.001\}$ for the JHT (left) and KF (right) finite difference schemes for Lorentz medium, with $\nu=1$. 


\section{Analysis of Dispersion and Phase Error}

The numerical approximation of time-dependent wave problems introduces errors which involve dissipation, dispersion, and anisotropy. The attenuation of the amplitude of the plane wave is referred to as dissipation. The numerical model produces waves that propagate at incorrect speeds. The dependence of the velocity of propagation of the numerical sinusoidal waves on frequency is termed as dispersion, while the dependence of the velocity upon direction is referred to as numerical anisotropy.

A dispersive model admits plane wave solutions of the form $\mathrm{e}^{\mathrm{i}(\omega t-\vec{k} \cdot \vec{x})}$ for which the speed of propagation, governed by the wave number $\vec{k}$, is not independent of the frequency $\omega$. For time-harmonic waves, numerical dispersion results in the creation of a numerical phase velocity error, or phase error, in the solution. This is due to the incorrect modeling of the sinusoidal behavior of the propagating wave, for example, the piecewise polynomial approximation of a finite element method does not exactly match a sine or cosine function. Dispersion is present in numerical approximation methods such as finite difference/finite element methods even in the absence of any dispersion in the actual media. Errors are cumulative, thus as waves propagate over long distances the numerical solution becomes corrupted and may completely deviate from the correct solution.

\subsubsection{Free Space}

Substituting a solution of the form

$$
e(t, z)=\mathrm{e}^{\mathrm{i}(k z-\omega t)}
$$

into equation (2.8) results in the dispersion relation of the continuous model in free space being

$$
k_{\mathrm{EX}}^{\mathrm{V}}(\omega)=\frac{\omega}{\mathrm{c}},
$$

where $k_{\mathrm{EX}}^{\mathrm{V}}$ denotes the wavenumber in free space (where $\mathrm{V}$ in the superscript denotes "vacuum") for the exact equations (EX in the subscript denotes "exact"). To determine the dispersion relation for the discretized model using the finite element method, we substitute

$$
X_{j}^{n}=\left[\begin{array}{c}
e_{j}^{n} \\
d_{j}^{n}
\end{array}\right]=\left[\begin{array}{c}
\tilde{e} \\
\tilde{d}
\end{array}\right] \mathrm{e}^{\mathrm{i}\left(k_{\Delta} j \Delta z-\omega n \Delta t\right)},
$$

where $k_{\Delta}$ is the numerical wave number, into the discrete equation (4.3). Using the identities (4.4), (4.5) as well as the following two trignometric identities

$$
\begin{aligned}
& \mathrm{e}^{-\mathrm{i} \omega \Delta t / 2}+\mathrm{e}^{\mathrm{i} \omega \Delta t / 2}=2 \cos (\omega \Delta t / 2), \\
& \mathrm{e}^{-\mathrm{i} \omega \Delta t / 2}-\mathrm{e}^{\mathrm{i} \omega \Delta t / 2}=-2 \mathrm{i} \sin (\omega \Delta t / 2) .
\end{aligned}
$$

we obtain the linear system

$$
\mathcal{A} \tilde{\mathcal{X}}=0
$$

with

$$
\mathcal{A}=\left[\begin{array}{cc}
2 \mathrm{i} \sin \left(\frac{\omega \Delta t}{2}\right) & \Delta t \cos \left(\frac{\omega \Delta t}{2}\right) \\
\frac{4 \Delta t}{\Delta z} \sin ^{2}\left(\frac{k_{\Delta} \Delta z}{2}\right) \cos \left(\frac{\omega \Delta t}{2}\right) & -\frac{\Delta z}{3 c^{2}}\left(3-2 \sin ^{2}\left(\frac{k_{\Delta} \Delta z}{2}\right)\right) 2 \mathrm{i} \sin \left(\frac{\omega \Delta t}{2}\right)
\end{array}\right]
$$


and

$$
\tilde{\mathcal{X}}=[\tilde{e}, \tilde{d}]^{T} .
$$

Setting the determinant of $\mathcal{A}=0$ we obtain a relation between $k_{\Delta}$ and $\omega$, which is the numerical dispersion relation for the finite element scheme in free space. This relation is

$$
\sin ^{2}\left(k_{\Delta} \Delta z / 2\right)=\left(\frac{2}{3}+\frac{\nu^{2} \cos ^{2}(\omega \Delta t / 2)}{\sin ^{2}(\omega \Delta t / 2)}\right)^{-1} .
$$

Solving for $k_{\Delta}$ in the above we get

$$
k_{\Delta}=k_{\mathrm{FE}}^{\mathrm{V}}(\omega)=\frac{2}{\Delta z} \sin ^{-1}\left(\frac{1}{\sqrt{\frac{2}{3}+\frac{\nu^{2} \cos ^{2}(\omega \Delta t / 2)}{\sin ^{2}(\omega \Delta t / 2)}}}\right) .
$$

The dispersion relation for the FDTD scheme is given to be

$$
\sin \left(k_{\Delta} \Delta z / 2\right)=\frac{\sin (\omega \Delta t / 2)}{\nu},
$$

which implies that

$$
k_{\Delta}=k_{\mathrm{FD}}^{\mathrm{V}}(\omega)=\frac{2}{\Delta z} \sin ^{-1}\left[\frac{\sin (\omega \Delta t / 2)}{\nu}\right] .
$$

We will define the phase error as

$$
\Phi(\omega \Delta t)=\left|\frac{k_{\mathrm{EX}}(\omega \Delta t)-k_{\Delta}(\omega \Delta t)}{k_{\mathrm{EX}}(\omega \Delta t)}\right|
$$

where for the finite element scheme in a vacuum, we have $k_{\Delta}=k_{\mathrm{FE}}^{\mathrm{V}}$ as defined in (5.7), whereas for the FDTD schemes we have $k_{\Delta}=k_{\text {FD }}^{\mathrm{V}}$ as defined in (5.9).

In Figures 10 and 11 we plot the phase error in a vacuum for the finite element scheme with the theta method $(\nu=\sqrt{1 / 2}, 1,4,16)$ and for the FDTD scheme $(\nu=$ $0.6, \sqrt{1 / 2}, \sqrt{2 / 3}, 1)$, respectively.

To see why $\nu \approx .7$ has the least dispersion for FEM (see plot on the right in Figure 10) and why $\nu \approx 1$ has the least dispersion for FDTD (see plot on the right in Figure 11), it is helpful to plot the relations in equations (5.6) and (5.8) versus the continuous model values. We define

$$
\gamma_{\mathrm{EX}}^{2}:=\sin ^{2}\left(\frac{\omega d t}{2 \nu}\right)
$$

where we have substituted $k_{\mathrm{EX}}^{\mathrm{V}} \Delta z=\nu \omega \Delta t$. We similarly define $\gamma_{\mathrm{FE}}^{2}=\sin ^{2}\left(k_{\mathrm{FE}}^{\mathrm{V}} \Delta z / 2\right)$ and $\gamma_{F D}^{2}=\sin ^{2}\left(k_{\mathrm{FD}}^{\mathrm{V}} \Delta z / 2\right)$ using the definitions in (5.7) and (5.9), respectively. Figure 12 displays plots each of these $\gamma^{2}(\omega \Delta t)$ functions for various values of $\nu$. For the continuous model (left plot), $\nu$ has the effect of moving the location of the maximum value, $\gamma^{2}=1$. For the finite difference case (right plot) the location of the maximum does not change, although the value of the maximum does. For $\nu=1$ the curve coincides exactly with the continuous case. For the finite element method (middle plot) the location of the maximum 

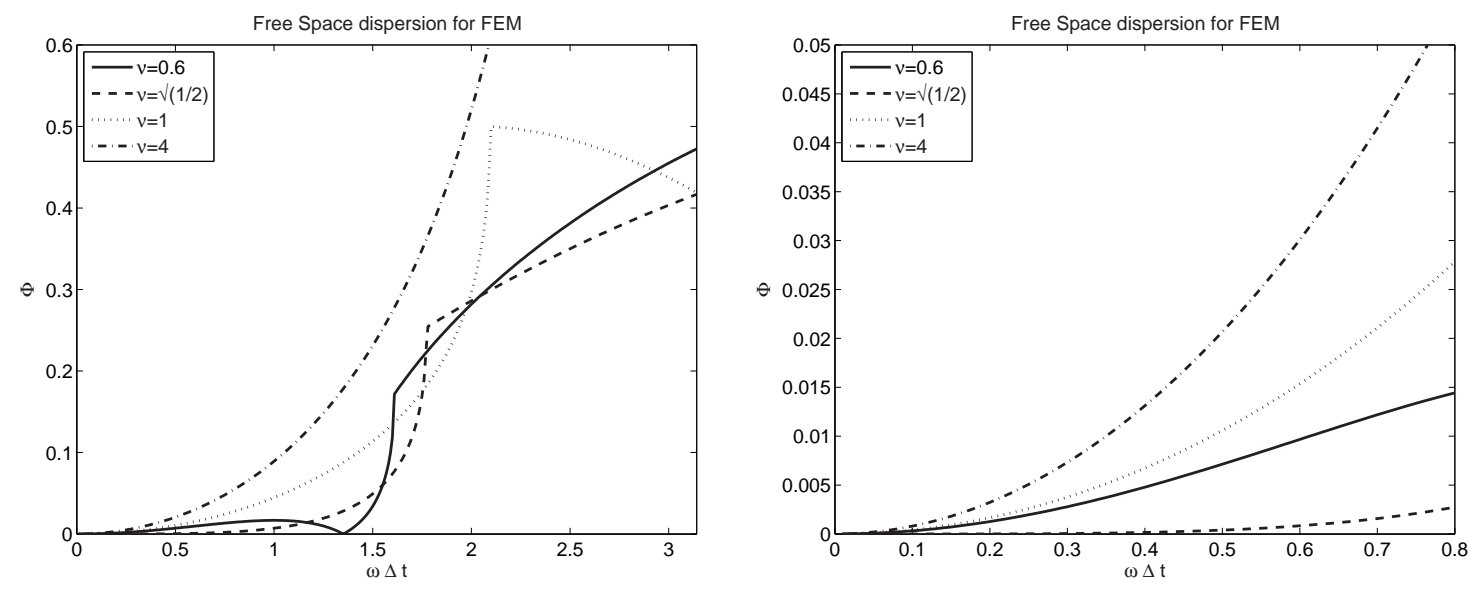

Figure 10: Plot of the phase error $\Phi$ versus $\omega \Delta t$ for the finite element scheme in freespace with $\nu=\{\sqrt{1 / 2}, 1,4,16\}$. For $\nu=\sqrt{1 / 2}$, FEM has the least dispersion. (Right plot is zoom of left plot.)
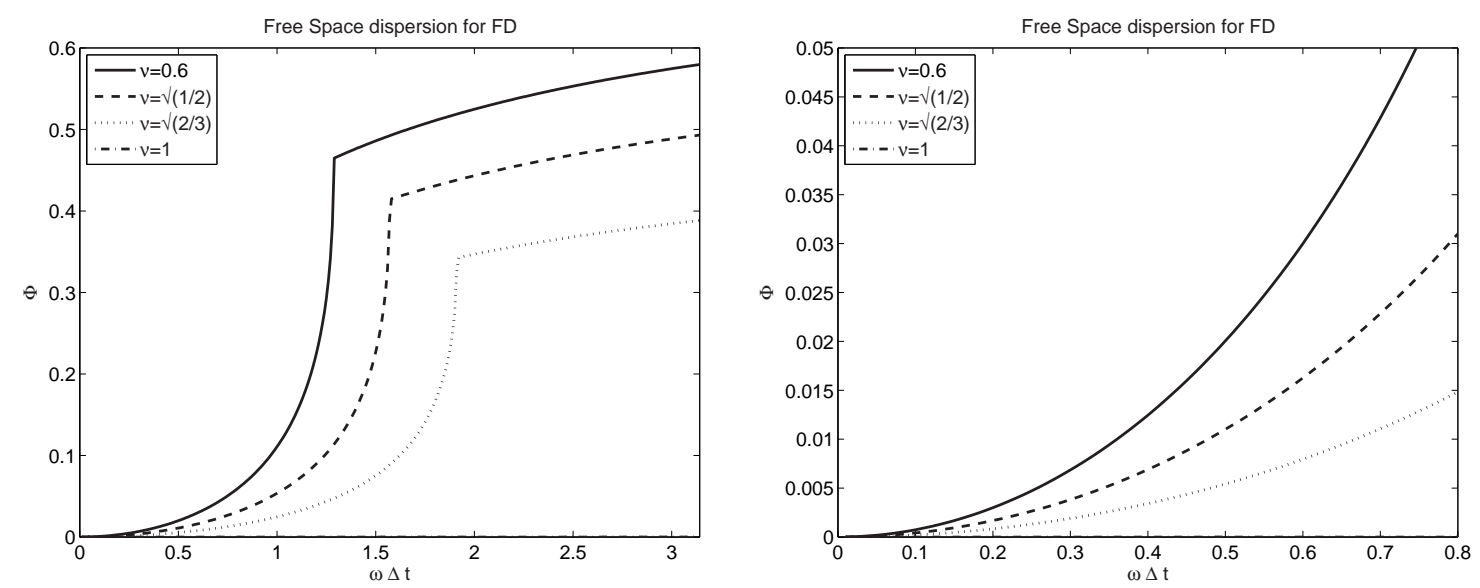

Figure 11: Plot of the phase error $\Phi$ versus $\omega \Delta t$ for the finite difference scheme in freespace with $\nu=\{1, \sqrt{2 / 3}, \sqrt{1 / 2}, .6\}$. For $\nu=1$ FDTD has zero dispersion. (Right plot is zoom of left plot.)

does not change, nor does the value. However, as this value is fixed at 1.5 , it will never coincide exactly with the continuous case for any value of $\nu$.

To determine the best value of $\nu$ for the finite element method, we first note that $\gamma_{\mathrm{EX}}^{2}$ can be expanded as

$$
1 / 4 \frac{\omega^{2} \Delta t^{2}}{\nu^{2}}-1 / 48 \frac{\omega^{4} \Delta t^{4}}{\nu^{4}}+\frac{1}{1440} \frac{\omega^{6} \Delta t^{6}}{\nu^{6}}+O\left(\omega^{8} \Delta t^{8}\right) .
$$

As both the expansions of $\gamma_{\mathrm{FD}}$ and $\gamma_{\mathrm{FE}}$ match up to the second order coefficient, it is the fourth order Taylor coefficient that determines how well the discretization method matches 

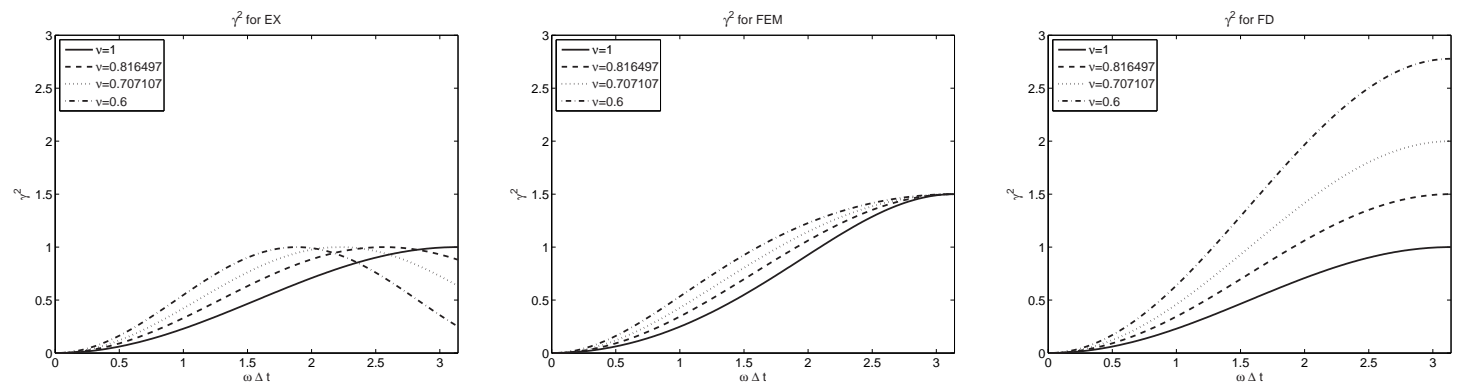

Figure 12: Plot of the $\gamma^{2}$ versus $\omega \Delta t$ for the continuous model, the finite elment scheme, and the finite difference scheme (left to right).

the continuous model. For the finite difference method we have

$$
c_{4}^{\mathrm{FD}}=-1 / 48 \nu^{-2},
$$

whereas for the finite element method we have

$$
c_{4}^{\mathrm{FE}}=1 / 24 \frac{-1+\nu^{2}}{\nu^{4}}
$$

For $\nu=1, c_{4}^{\mathrm{FD}}=c_{4}^{\mathrm{EX}}=1 / 48$, but $c_{4}^{\mathrm{FE}}=0$. If we solve $\left(c_{4}^{\mathrm{EX}}-c_{4}^{\mathrm{FE}}\right)(\nu)=0$ we find that $\nu=\sqrt{1 / 2}$ is the value for which the finite element method most closely matches the continuous model.

In Figure 13 we plot the fourth-order Taylor coefficient of $\sin (k \Delta z / 2)$ as a function of $\nu$ for $k=\left\{k_{\mathrm{EX}}^{\mathrm{V}}, k_{\mathrm{FE}}^{\mathrm{V}}, k_{\mathrm{FD}}^{\mathrm{V}}\right\}$, i.e., the $k$ values for the continuous model, the finite element scheme and finite difference scheme, respectively. As mentioned above, for $\nu=\sqrt{1 / 2}$ the coefficient corresponding to the finite element scheme is equal to that of the value corresponding to the exact $k$. This suggests that least dispersion will be achieved in the finite element scheme when $\nu=\sqrt{1 / 2}$. For $\nu=1$ the coefficient corresponding to the finite difference scheme is equal to that of the value corresponding to $k_{\mathrm{EX}}^{\mathrm{V}}$. It is known that this is the value of $\nu$ for which finite difference schemes exhibit least dispersion. Further, for $\nu=\sqrt{2 / 3}$ the coefficient corresponding to the finite element scheme is equal to that of the finite difference scheme. The dispersion curves are nearly identical for the two schemes when $\nu=\sqrt{2 / 3}$. The plots of $\gamma^{2}$ for these values of $\nu$ are displayed in Figure 14.

In Figures 15 and 16 we plot the phase velocity $v_{p}$ (scaled by $\frac{1}{\mathrm{c}}$ ) versus $\omega \Delta t$ for the finite element scheme with the theta method and the phase error for the FDTD schemes, respectively. 


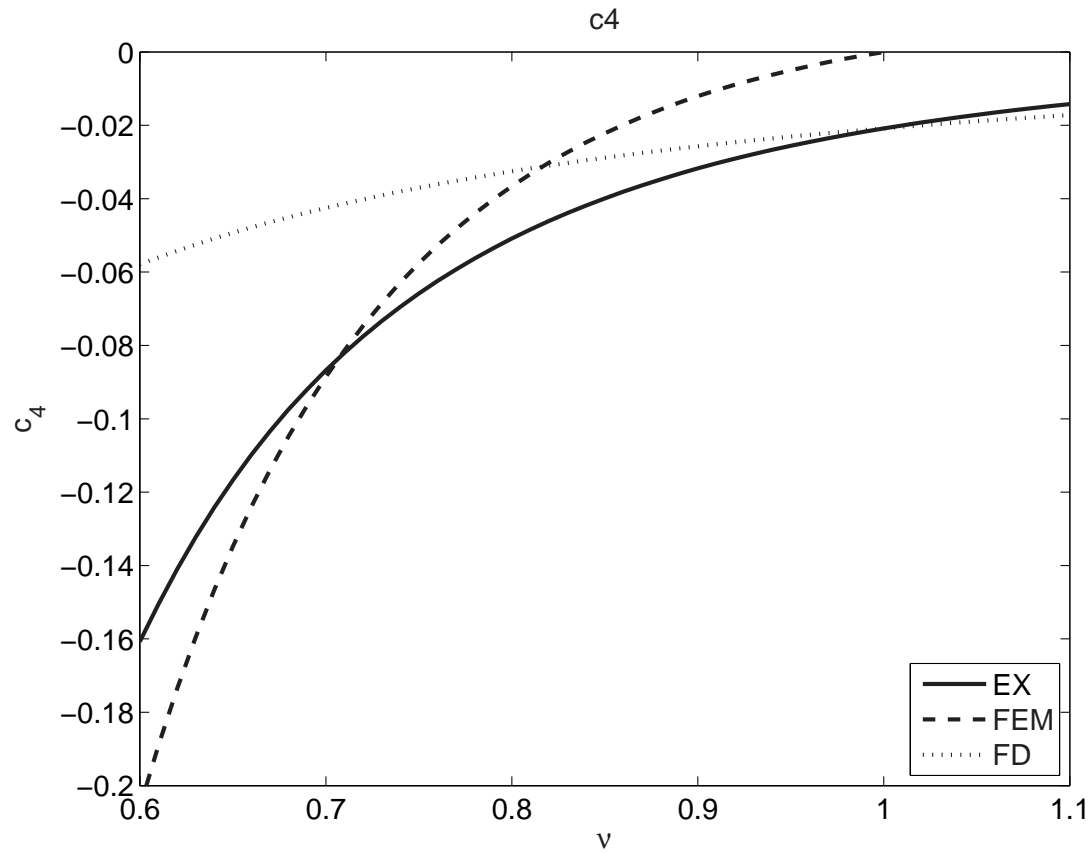

Figure 13: Value of the 4 th order Taylor coefficient of $\sin ^{2}\left(\frac{k \Delta z}{2}\right)$ for $k=\left\{k_{\mathrm{EX}}^{\mathrm{V}}, k_{\mathrm{FE}}^{\mathrm{V}}, k_{\mathrm{FD}}^{\mathrm{V}}\right\}$.
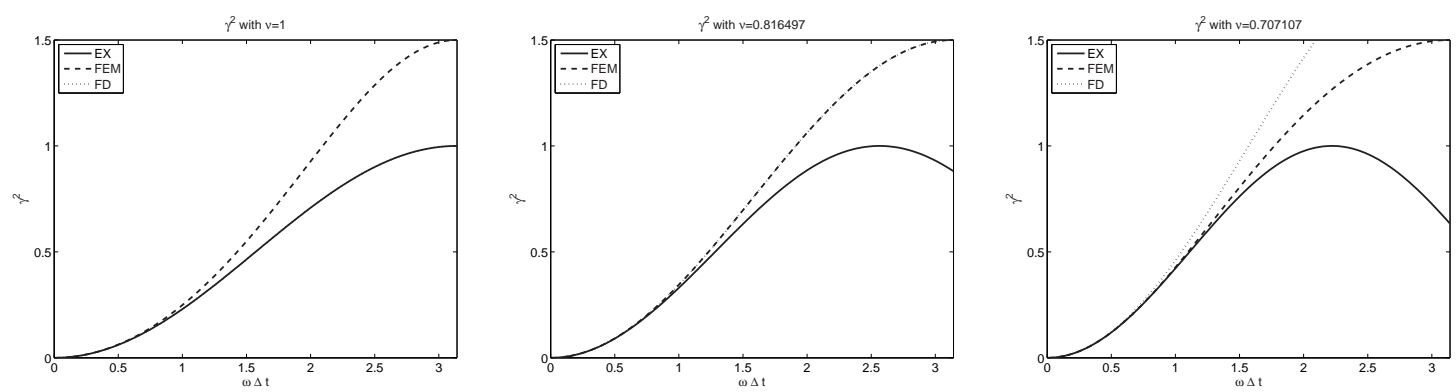

Figure 14: Plot of the $\gamma^{2}$ versus $\omega \Delta t$ for the continuous model, the finite elment scheme, and the finite difference scheme (Left plot uses $\nu=1$, center plot uses $\nu=\sqrt{2 / 3}$, right plot uses $\nu=\sqrt{1 / 2}$ ). 

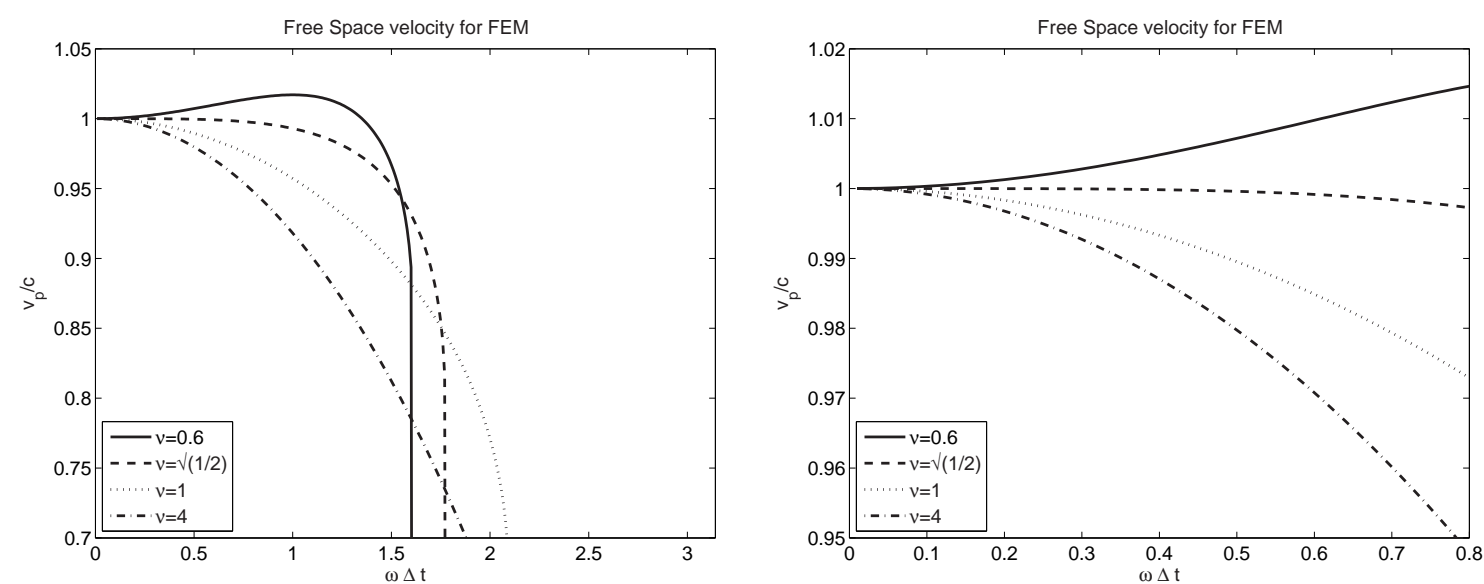

Figure 15: Plot of the velocity $v_{p}$ (scaled by $\frac{1}{\mathrm{c}}$ ) versus $\omega \Delta t$ for the finite element scheme in freespace with $\nu=\{\sqrt{1 / 2}, 1,4,16\}$. For $\nu=\sqrt{1 / 2}$, FEM has the velocity closest to one. Note that for $\nu<\sqrt{1 / 2}$, the velocity $v_{p}$ is greater than $c$.
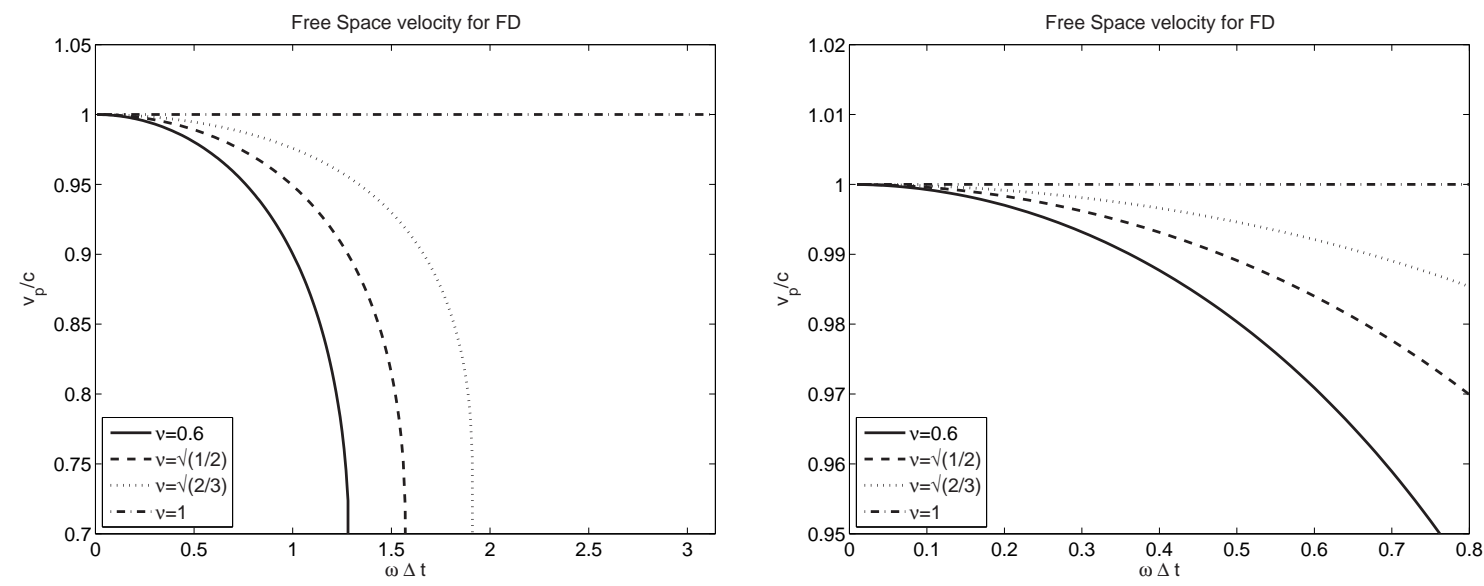

Figure 16: Plot of the velocity $v_{p}$ (scaled by $\frac{1}{c}$ ) versus $\omega \Delta t$ for the finite difference scheme in freespace with $\nu=\{1, \sqrt{2 / 3}, \sqrt{1 / 2}, .6\}$. For $\nu=1$ FDTD always has velocity one. 


\subsubsection{Debye Media}

The dispersion relation for the continuous Debye model is given by

$$
k_{\mathrm{EX}}^{\mathrm{D}}(\omega)=\frac{\omega}{\mathrm{c}} \sqrt{\epsilon_{r}^{\mathrm{D}}(\omega)} .
$$

where

$$
\epsilon_{r}^{\mathrm{D}}(\omega)=\frac{\epsilon_{s} \lambda-\mathrm{i} \omega}{\lambda-\mathrm{i} \omega}
$$

is the relative complex permittivity of the Debye medium, $\lambda=1 / \tau$. To determine the numerical dispersion relation for the finite element method described as Method 1 in Section 3.2.1 applied to a Debye media, we substitute

$$
X_{j}^{n}=\left[\begin{array}{c}
e_{j}^{n} \\
p_{j}^{n} \\
d_{j}^{n}
\end{array}\right]=\left[\begin{array}{c}
\tilde{e} \\
\tilde{p} \\
\tilde{d}
\end{array}\right] \mathrm{e}^{\mathrm{i}\left(k_{\Delta} j \Delta z-\omega n \Delta t\right)}
$$

in the discrete equations (3.13) (with $J=0$ ). We assume again that $\epsilon_{\infty}=1$. As in the case of free space, we obtain a homogeneous system of the type $\mathcal{A} \tilde{\mathcal{X}}=0$. We then set $\operatorname{det}(\mathcal{A})=0$ to obtain a relation between the numerical wavenumber $k_{\Delta}$ and the frequency $\omega$. Solving for $k_{\Delta}$ in this relation, and writing in a form comparable to (5.15), we obtain the numerical dispersion relation for the FEM1 scheme applied to Debye media to be

$$
k_{\Delta}=k_{\mathrm{FE}}^{\mathrm{D}}(\omega)=\frac{2}{\Delta z} \sin ^{-1}\left[\frac{\omega_{\Delta}}{c} \frac{\Delta z}{2} \sqrt{\epsilon_{r, \mathrm{FE}}^{\mathrm{D}}}\right],
$$

with the discrete relative complex permittivity given by

$$
\epsilon_{r, \mathrm{FE}}^{\mathrm{D}}=\frac{\epsilon_{s, \Delta} \lambda_{\Delta}-\mathrm{i} \omega_{\Delta}}{\lambda_{\Delta}-\mathrm{i} \omega_{\Delta}}
$$

where the discrete medium parameters are

$$
\begin{aligned}
\epsilon_{s, \Delta} & =\frac{\epsilon_{s}}{\alpha^{2} \beta^{2}} \\
\lambda_{\Delta} & =\lambda \cos (\omega \Delta t / 2) \beta^{2} \alpha^{3} \\
\omega_{\Delta} & =\omega s_{\omega} \alpha
\end{aligned}
$$

and

$$
\begin{aligned}
s_{\omega} & =\frac{\sin (\omega \Delta t / 2)}{\omega \Delta t / 2} \\
\alpha & =\left(\frac{2 \sin ^{2}(\omega \Delta t / 2)}{3 \nu^{2}}+\cos ^{2}(\omega \Delta t / 2)\right)^{-1 / 2} \\
\beta & =\left(\frac{2 \epsilon_{s} \sin ^{2}(\omega \Delta t / 2)}{3 \nu^{2}}+\cos ^{2}(\omega \Delta t / 2)\right)^{1 / 2} .
\end{aligned}
$$

To determine the numerical dispersion relation for the finite element scheme described in Method 2 in Section (3.2.1), we substitute (4.13) in the discrete equations (3.17). Following 
the procedure discussed above we obtain the numerical dispersion relation for the FEM1 scheme applied to Debye media to be

$$
\sin ^{2}\left(k_{\Delta} \Delta z / 2\right)=\alpha^{2}\left(\frac{2 \sin (\eta) i-\lambda \cos (\eta) \epsilon_{s} \Delta t+\frac{1}{4} h_{\tau}^{3} \cos (\eta) \epsilon_{d}}{2 \sin (\eta) i-\lambda \cos (\eta) \epsilon_{s} \Delta t \beta^{2} \alpha^{2}+\frac{1}{6} h_{\tau}^{3} \cos (\eta) \epsilon_{d} \alpha^{2}}\right),
$$

where $\eta=\frac{\omega \Delta t}{2}, \epsilon_{d}=\epsilon_{s}-1$ and $h_{\tau}=\Delta t / \tau$. If we neglect terms in $h_{\tau}^{3}$, then the expression (5.26) reduces to (5.18). Thus, for small $h_{\tau}$, both the finite element methods have the same numerical dispersion relations. From the section on stability analysis we have seen that for low dissapation $h_{t}$ au needs to be about 0.001 for Debye media and 0.01 for Lorentz media. For these values of $h_{\tau}$ both finite element schemes produce the same dispersion graphs.

Both the FEM schemes misrepresent the continuous model parameters $\lambda$ and $\epsilon_{s}$ discretely as $\lambda_{\Delta}$ and $\epsilon_{s, \Delta}$, and misrepresent the frequency $\omega$ as $\omega_{\Delta}$. We note that as $\nu$ increases $\left(\nu>\sqrt{\epsilon_{s}}\right)$, the product $\alpha \beta \rightarrow 1$, and $\epsilon_{s, \Delta} \rightarrow \epsilon_{s}$. The discrete parameter $\lambda_{\Delta}$ for the finite element method is a function of the continuous model parameter $\epsilon_{s}$ via the quantity $\beta$. However, for the regime of interest, namely $\omega \Delta t$ small, and when $\nu$ is large $\left(\nu>\sqrt{\epsilon_{s}}\right), 2 \epsilon_{s} \sin ^{2}(\omega \Delta t / 2) / 3 \nu^{2}$ is dominated by $\cos ^{2}(\omega \Delta t / 2)$. Also the product $\alpha \beta \rightarrow 1$, $\alpha \rightarrow 1 / \cos (\omega \Delta / 2)$ and thus $\lambda_{\Delta} \rightarrow \lambda$. Finally for $\omega \Delta t$ small and $\nu$ large, $s_{\omega} \alpha \approx 1$ and thus $\omega_{\Delta} \rightarrow \omega$. Hence, the choice of the Courant number $\nu$ is important in maintaining low dispersion error in the discrete FEM.

We compare the FEM scheme for Debye media with the finite difference scheme presented in [18] and analyzed in [28] (for the Debye media, this method is again equivalent to the scheme in [20]). The numerical dispersion relation for this finite difference scheme applied to Debye media is given to be

$$
\begin{gathered}
k_{\Delta}=k_{\mathrm{FD}}^{\mathrm{D}}(\omega)=\frac{2}{\Delta z} \sin ^{-1}\left[\frac{\omega}{c} s_{\omega} \frac{\Delta z}{2} \sqrt{\epsilon_{r, \mathrm{FD}}^{\mathrm{D}}}\right], \\
\epsilon_{r, \mathrm{FD}}^{\mathrm{D}}=\frac{\epsilon_{s} \lambda_{\Delta}-i \omega_{\Delta}}{\lambda_{\Delta}-i \omega_{\Delta}} .
\end{gathered}
$$

If written in the form of (5.18), this would correspond to the following discrete representations of the continuous model parameters:

$$
\begin{aligned}
\epsilon_{s, \Delta} & =\epsilon_{s} \\
\lambda_{\Delta} & =\lambda \cos (\omega \Delta t / 2),
\end{aligned}
$$

and a representation of the frequency by

$$
\omega_{\Delta}=\omega s_{\omega}
$$

We compare the phase error for the finite element scheme applied to Debye media to phase error for the finite difference scheme. The phase error is plotted against values of $\omega \Delta t$ in the range $[0, \pi]$. We note that $\omega \Delta t=2 \pi / N_{\text {ppp }}$, where $N_{\text {ppp }}$ is the number of points per period, and is related to the number of points per wavelength $N_{\text {ppw }}$ via

$$
N_{\mathrm{ppw}}=\nu N_{\mathrm{ppp}}
$$


Thus, for $\nu \leq 1$, the number of points per wavelength is always less than or equal to the number of points per period, and conversely for $\nu>1$. Note that the number of points per wavelength in the range $[\pi / 4, \pi]$ is 8 to 2 points per period. We are more interested in the range $[0, \pi / 4]$ which involves more than 8 points per period (or equvivalently more than 8 points per wavelength).

To generate the plots below we have used the following values for the medium parameters:

$$
\begin{aligned}
\epsilon_{\infty} & =1 \\
\epsilon_{s} & =78.2 \\
\tau & =8.1 \times 10^{-12} \text { sec. }
\end{aligned}
$$

Figures 17-19 plot the phase error $\Phi$ versus $\omega \Delta t$ for the FEM scheme applied to the Debye model, with various values of $h_{\tau}$ and $\nu$. Figures 20-22 plot the same for the finite difference method.

Figures 23-25 plot the phase error $\Phi$ versus the frequency $\omega$ for the FEM scheme applied to the Debye model, with various values of $h_{\tau}$ and $\nu$. Figures 26-28 plot the same for the finite difference method. In these plots we can see that in the finite element scheme the phase error reduces as $\nu$ increases, even beyond 1 .

In Figure 31 plots of the phase error in the two finite element schemes described in Method 1 and Method 2 in Section 3.2.1 are shown with $\nu=1$ and $h_{\tau}=0.3$ (left) and $h_{\tau}=0.1$ (right). As can be seen in these plots the phase error in both the schemes is identical. A slight difference is noticeable in the case $h_{\tau}=0.3$, however for $h_{\tau}=0.1$ the two graphs are identical.

In Figures 32-37 we plot the real and imaginary parts of the relative complex permittivity for Debye media for the continuous equations (exact values), the finite element scheme with $\nu=\{\sqrt{1 / 2}, 1,4\}, h_{\tau}=\{0.1,0.01\}$, and finally the finite difference scheme with $\nu=1$ fixed and $h_{\tau}=\{0.1,0.01\}$. For $h_{\tau}=0.1$, as $\nu$ is increased the discrete permittivites of the finite element scheme approach the exact values. For $h_{\tau}=0.01$, the agreement of the discrete permittivities with the exact values is better than with $h_{\tau}=0.1$, for each value of $\nu$. For $\nu=4$ we see the best agreement of the discrete real and imaginary permittivities with the exact values. This is again seen in the plots of the discrete values of the parameters $\lambda$ and $\epsilon_{s}$ in Figures 39 and 38, respectively. In both these figures we see that the discrete parameters in the finite element scheme have better agreement with the exact values as $\nu$ is increased and $h_{\tau}$ is decreases. However the discrete value of the frequency $\omega$, Figure 40 , seems to have a better agreement with the exact value when $\nu=1$. For the finite difference scheme, the discrete parameters do not depend on the value of $\nu$. However as $h_{\tau}$ is decreased they agree better with the exact values. For the particular values tested here, it seems that the value of $\nu$ that correctly represents $\lambda$ will sufficiently model the complex permittivity. 

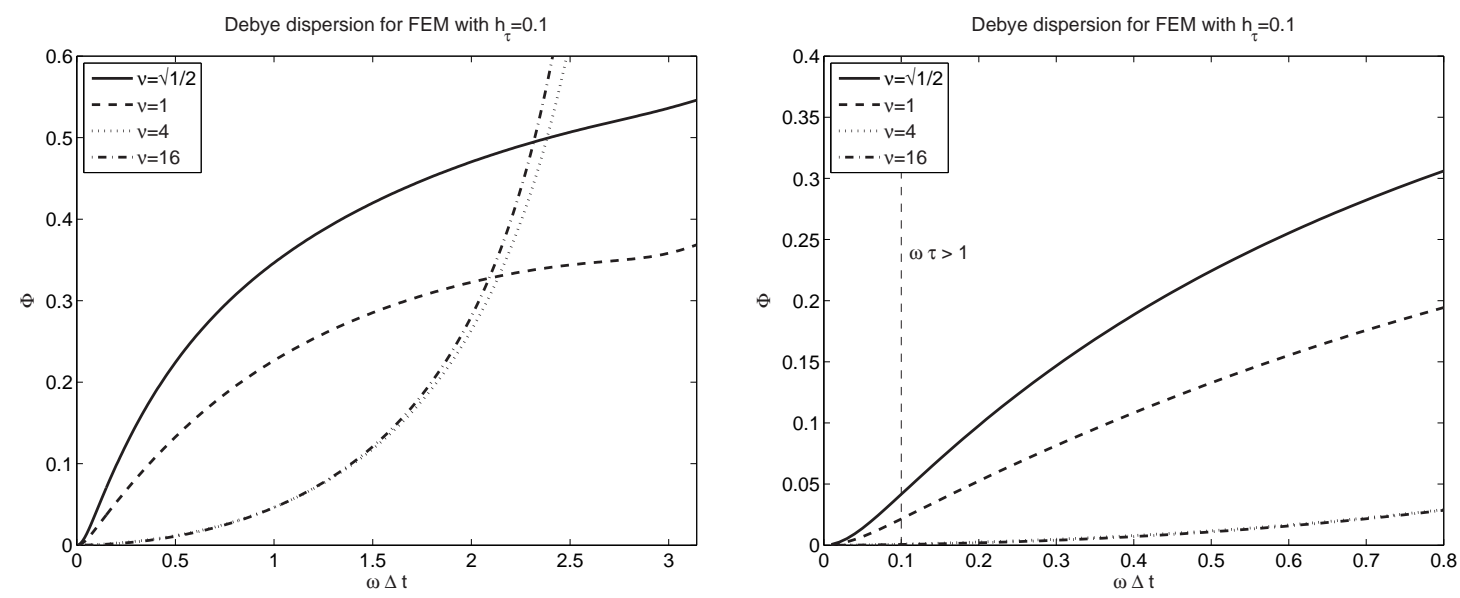

Figure 17: Plot of the phase error $\phi$ versus $\omega \Delta t$ for the finite element scheme of the Debye model with $\nu=\{\sqrt{1 / 2}, 1,4,16\}$ using $h_{\tau}=0.1$. (Right plot is zoom of left plot.)
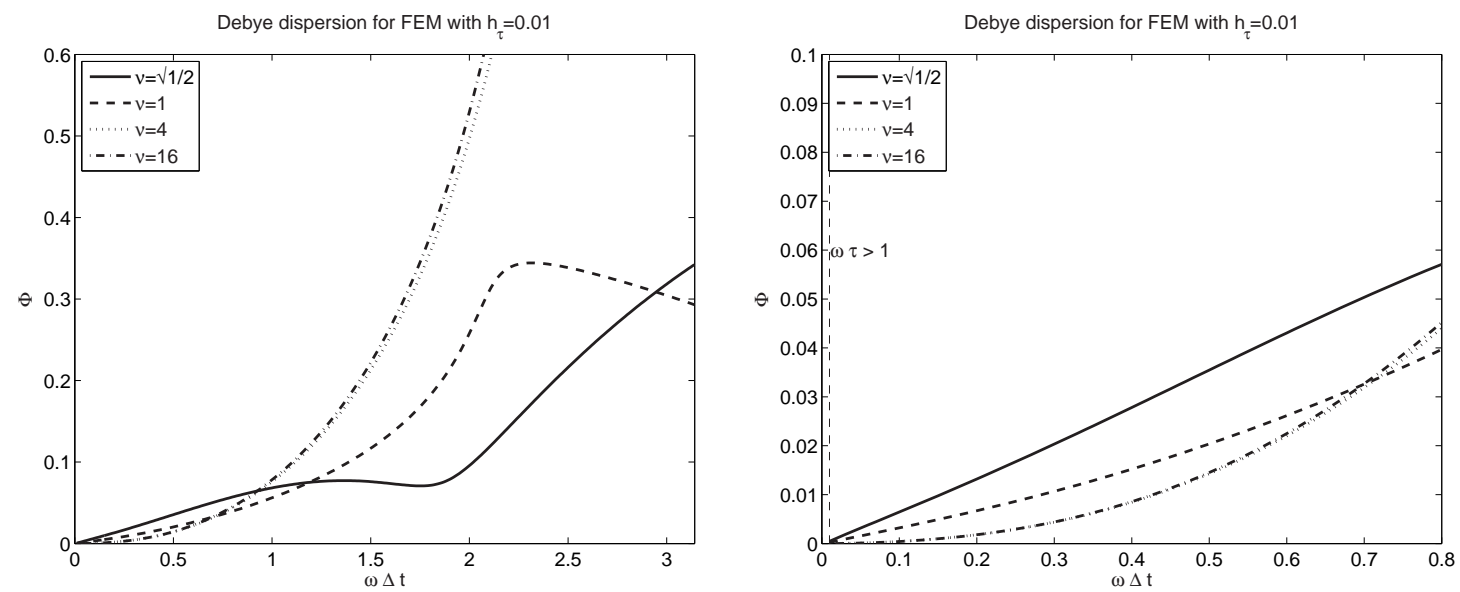

Figure 18: Plot of the phase error $\phi$ versus $\omega \Delta t$ for the finite element scheme of the Debye model with $\nu=\{\sqrt{1 / 2}, 1,4,16\}$ using $h_{\tau}=0.01$. (Right plot is zoom of left plot.) 

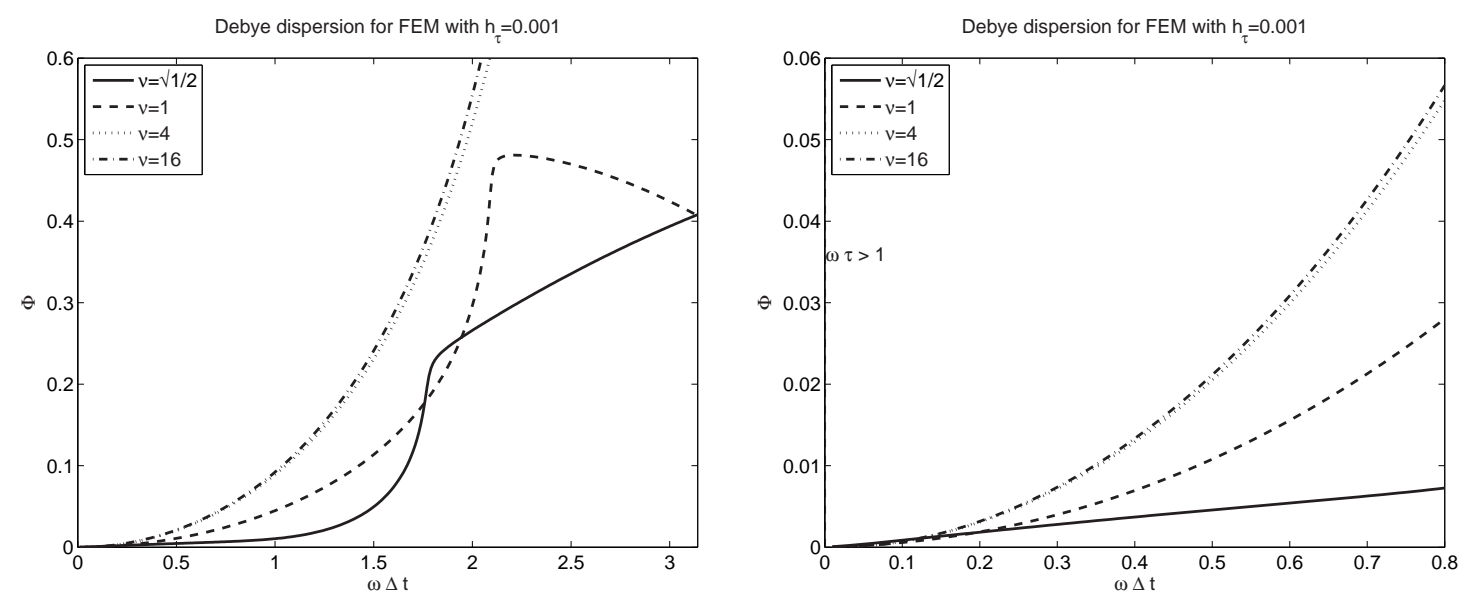

Figure 19: Plot of the phase error $\phi$ versus $\omega \Delta t$ for the finite element scheme of the Debye model with $\nu=\{\sqrt{1 / 2}, 1,4,16\}$ using $h_{\tau}=0.001$. (Right plot is zoom of left plot.)
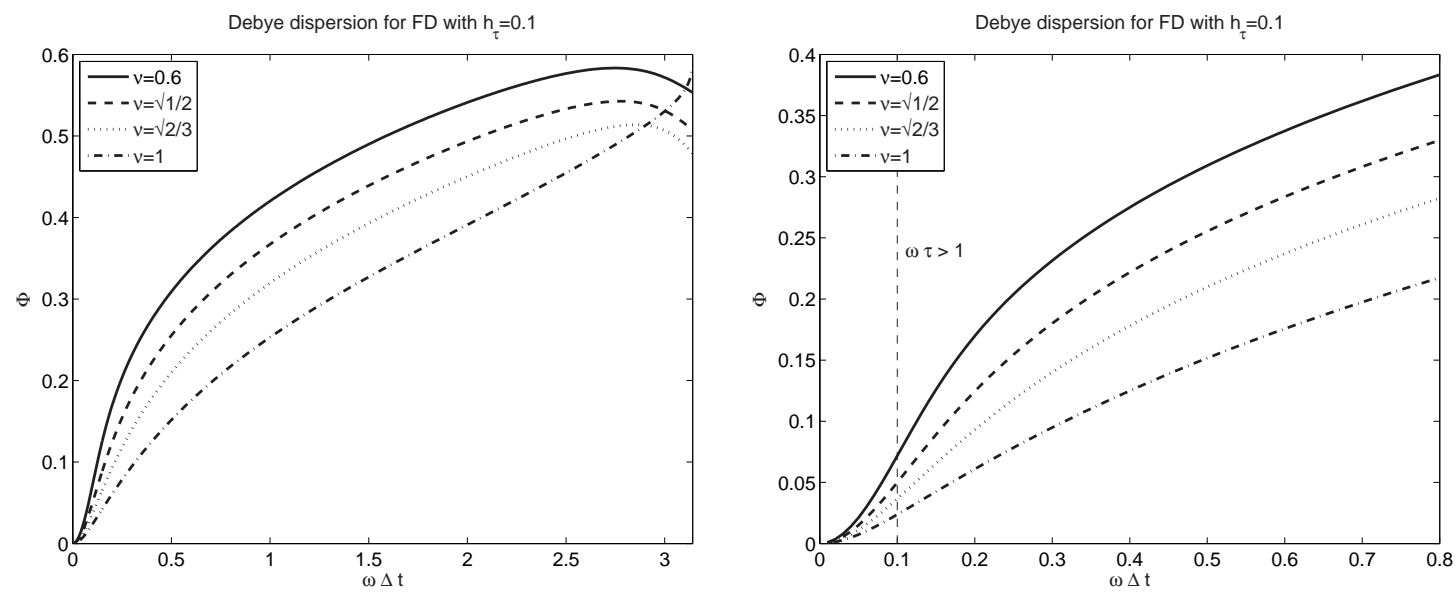

Figure 20: Plot of the phase error $\phi$ versus $\omega \Delta t$ for the finite difference scheme of the Debye model with $\nu=\{1, \sqrt{2 / 3}, \sqrt{1 / 2}, .6\}$ using $h_{\tau}=0.1$. (Right plot is zoom of left plot.) 

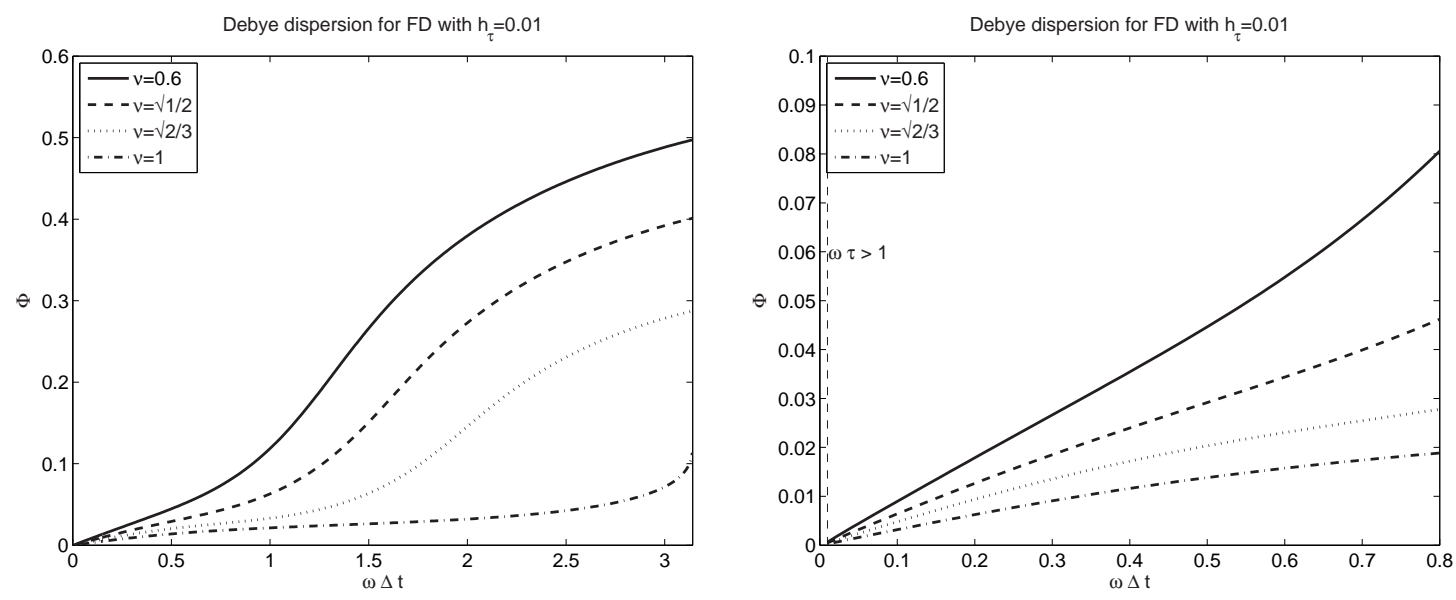

Figure 21: Plot of the phase error $\phi$ versus $\omega \Delta t$ for the finite difference scheme of the Debye model with $\nu=\{1, \sqrt{2 / 3}, \sqrt{1 / 2}, .6\}$ using $h_{\tau}=0.01$. (Right plot is zoom of left plot.)
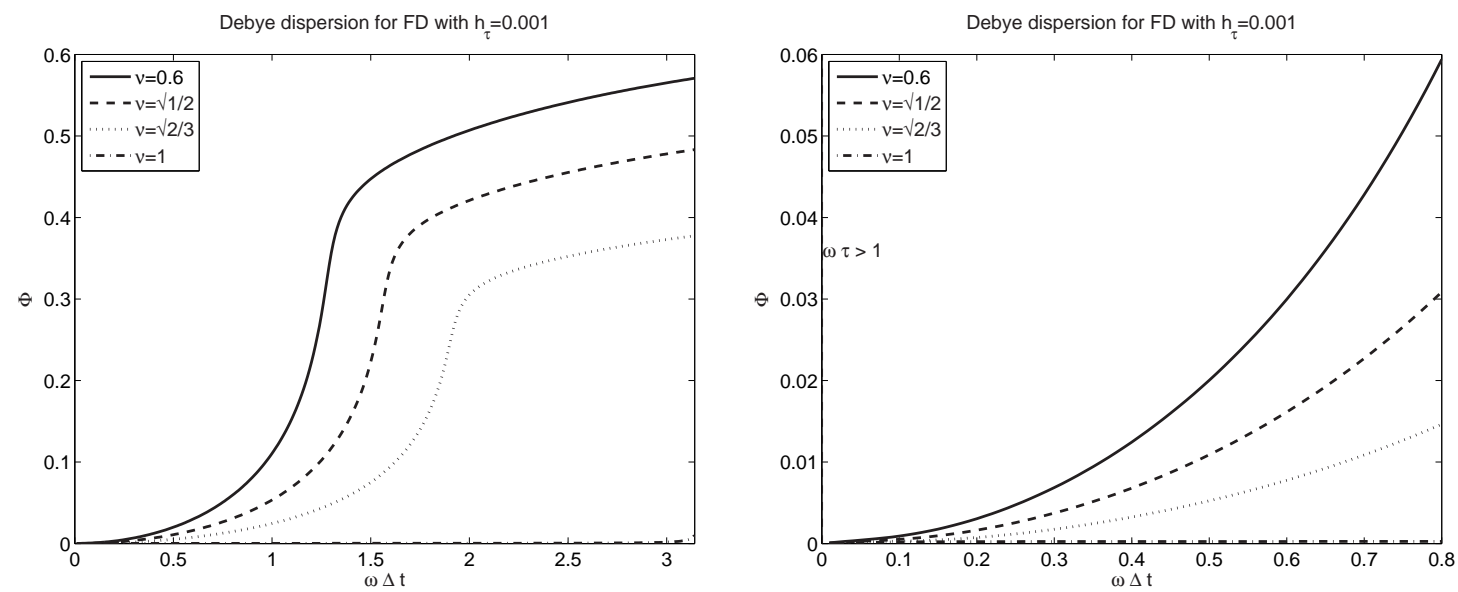

Figure 22: Plot of the phase error $\phi$ versus $\omega \Delta t$ for the finite difference scheme of the Debye model with $\nu=\{1, \sqrt{2 / 3}, \sqrt{1 / 2}, .6\}$ using $h_{\tau}=0.001$. (Right plot is zoom of left plot.) 

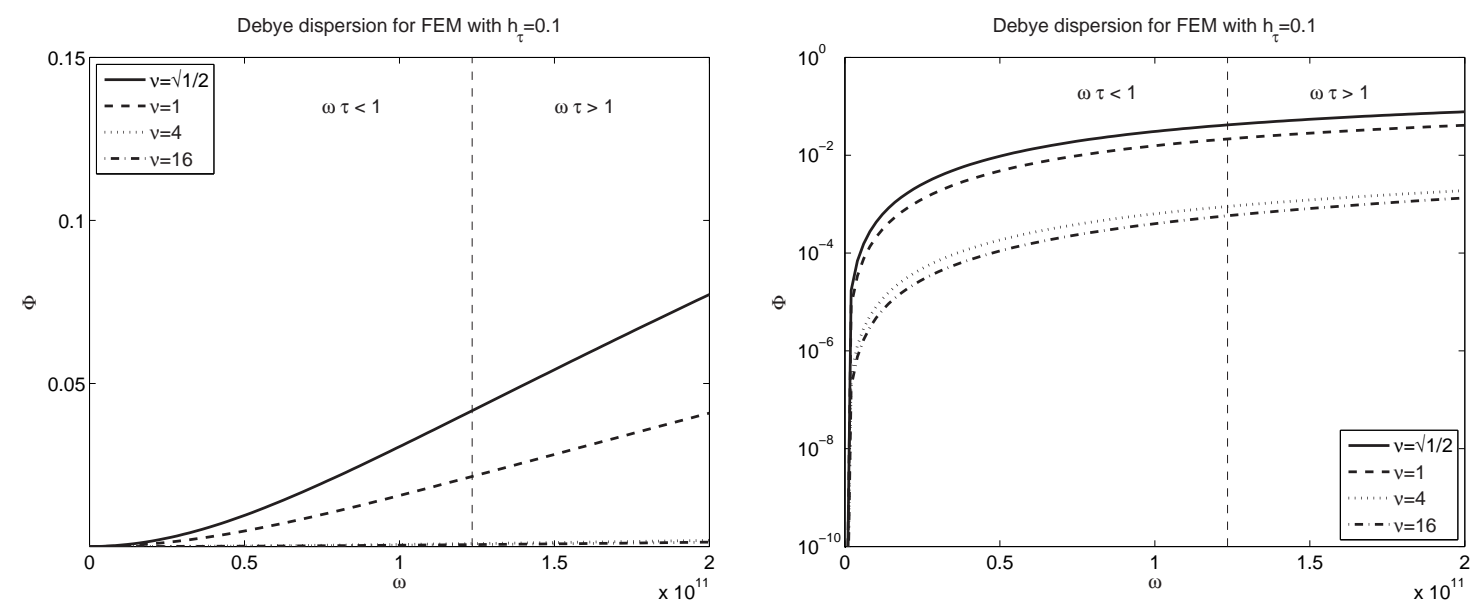

Figure 23: Plot of the phase error $\phi$ versus $\omega$ for the finite element scheme of the Debye model with $h_{\tau}=0.1$ using $\nu=\{\sqrt{1 / 2}, 1,4,16\}$. (Right plot is log of left plot.)
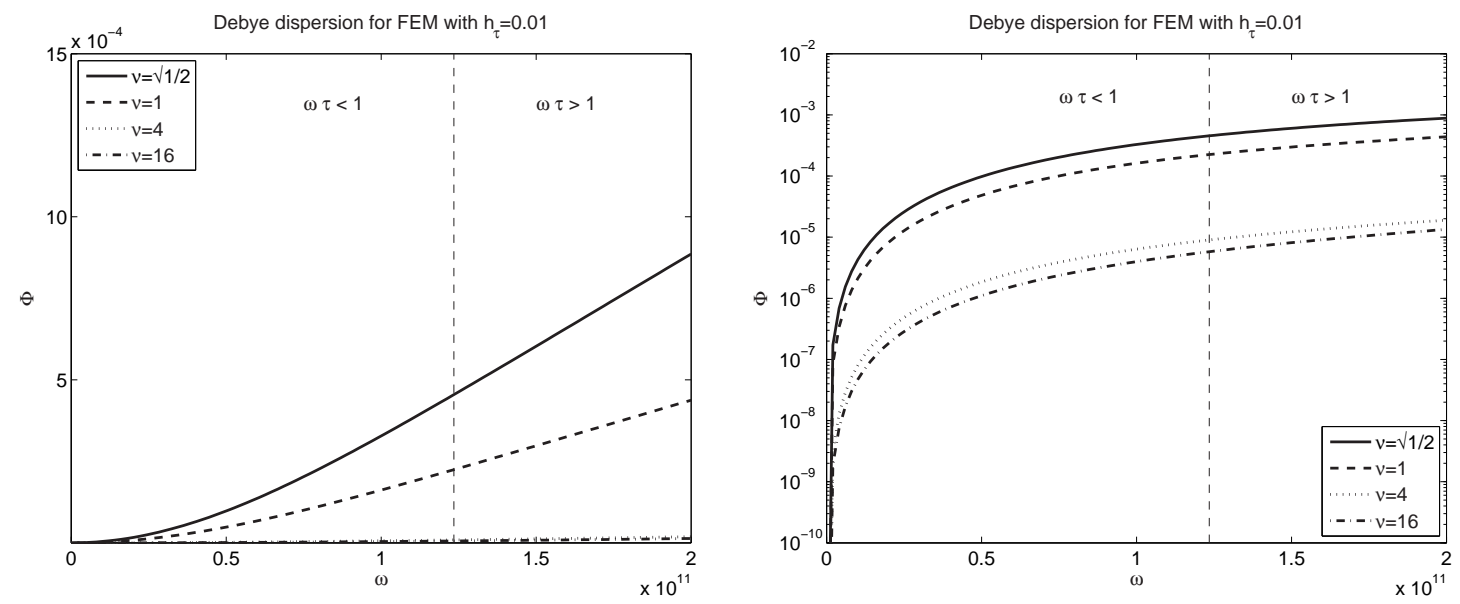

Figure 24: Plot of the phase error $\phi$ versus $\omega$ for the finite element scheme of the Debye model with $h_{\tau}=0.01$ using $\nu=\{\sqrt{1 / 2}, 1,4,16\}$. (Right plot is log of left plot.) 

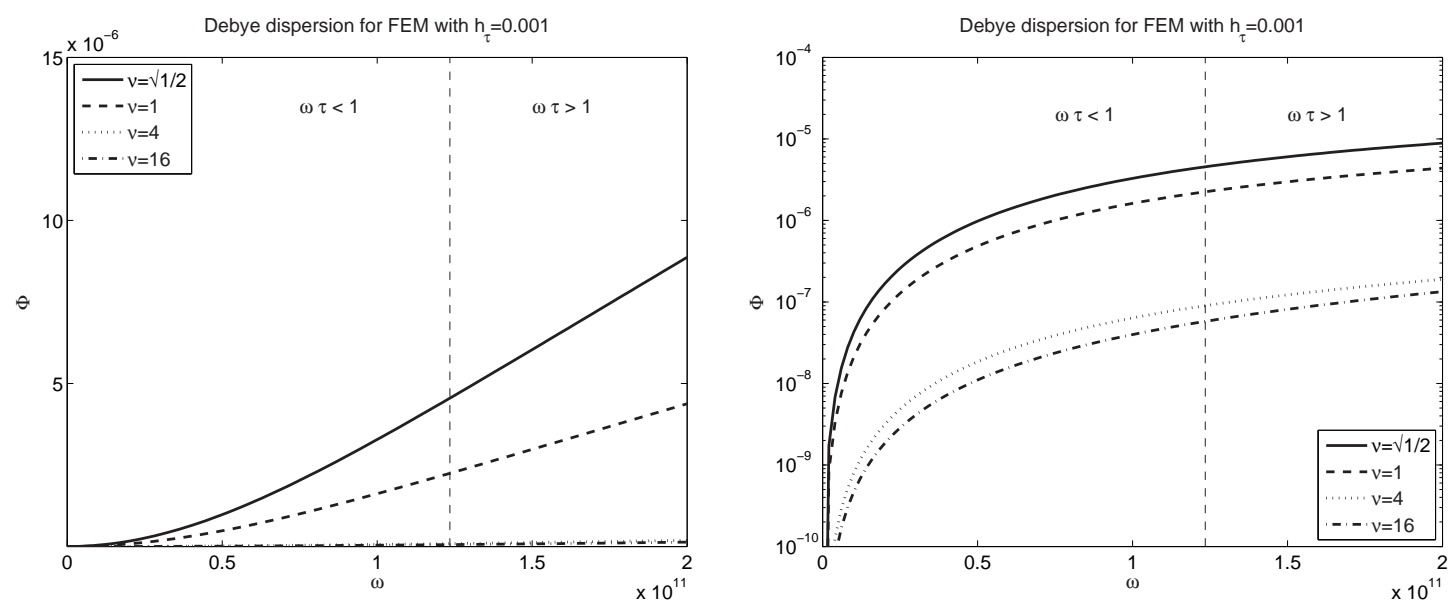

Figure 25: Plot of the phase error $\phi$ versus $\omega$ for the finite element scheme of the Debye model with $h_{\tau}=0.001$ using $\nu=\{\sqrt{1 / 2}, 1,4,16\}$. (Right plot is log of left plot.)
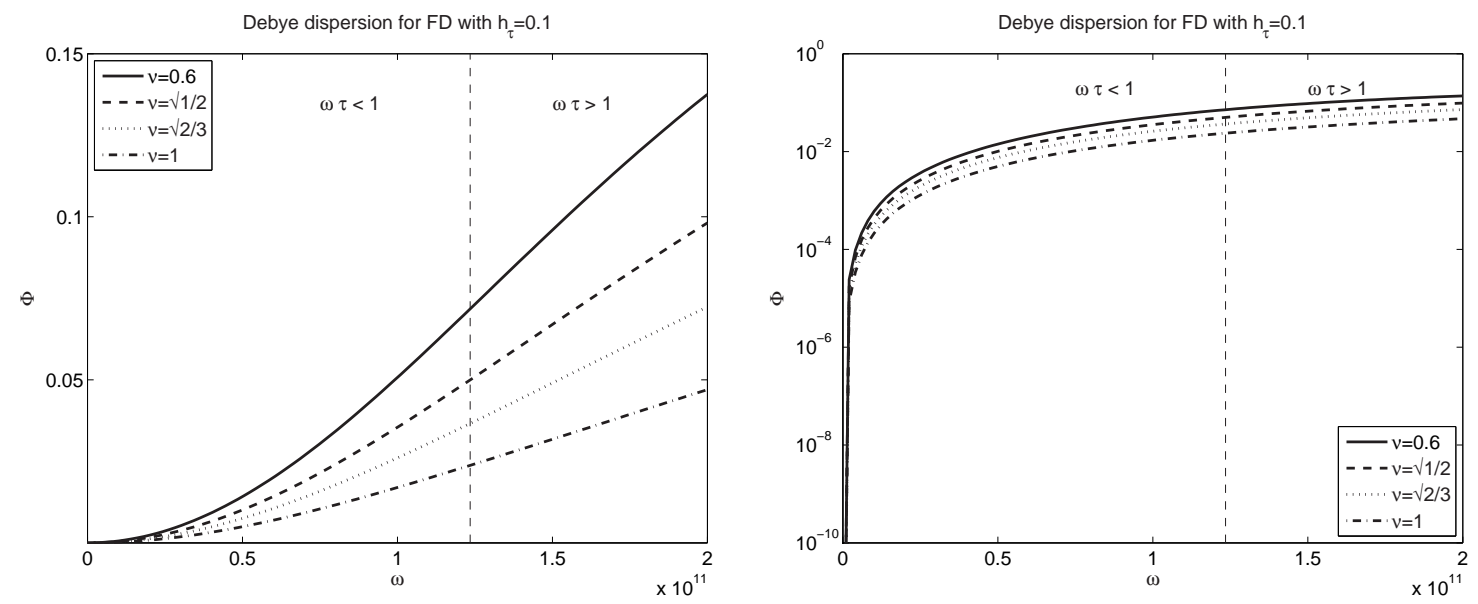

Figure 26: Plot of the phase error $\phi$ versus $\omega$ for the finite difference scheme of the Debye model with $h_{\tau}=0.1$ using $\nu=\{0.6, \sqrt{1 / 2}, \sqrt{2 / 3}, 1\}$. (Right plot is log of left plot.) 

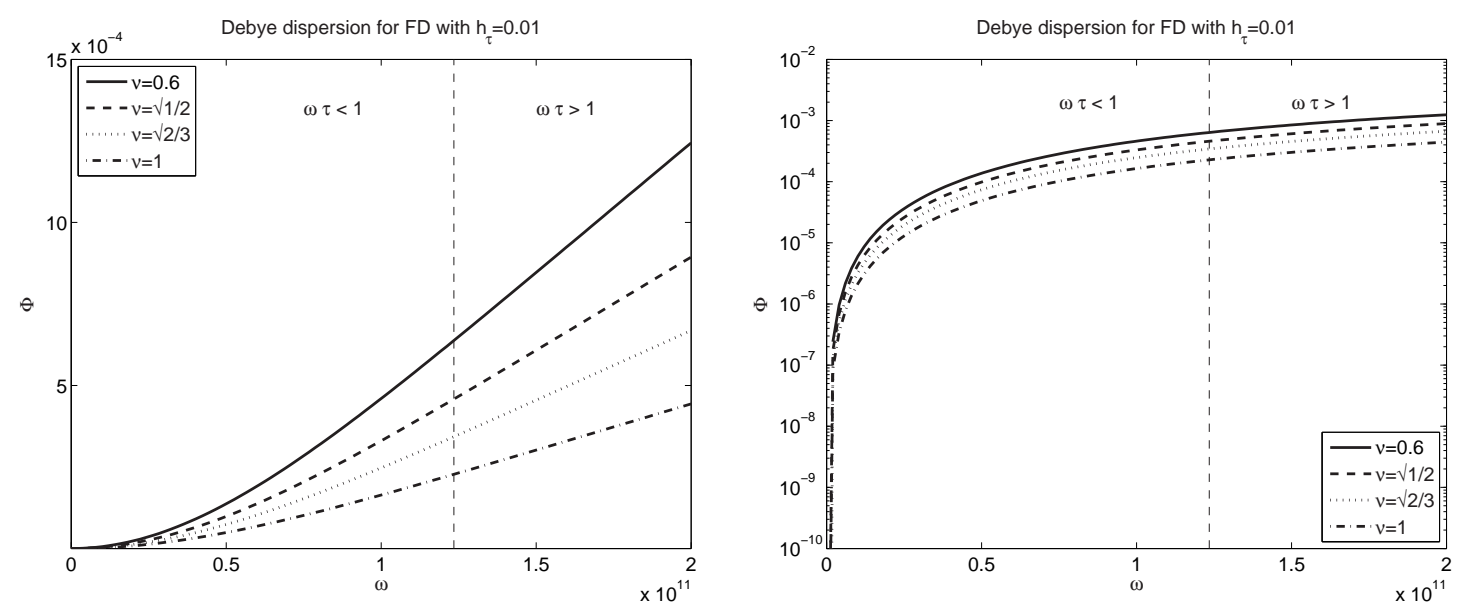

Figure 27: Plot of the phase error $\phi$ versus $\omega$ for the finite difference scheme of the Debye model with $h_{\tau}=0.01$ using $\nu=\{0.6, \sqrt{1 / 2}, \sqrt{2 / 3}, 1\}$. (Right plot is log of left plot.)
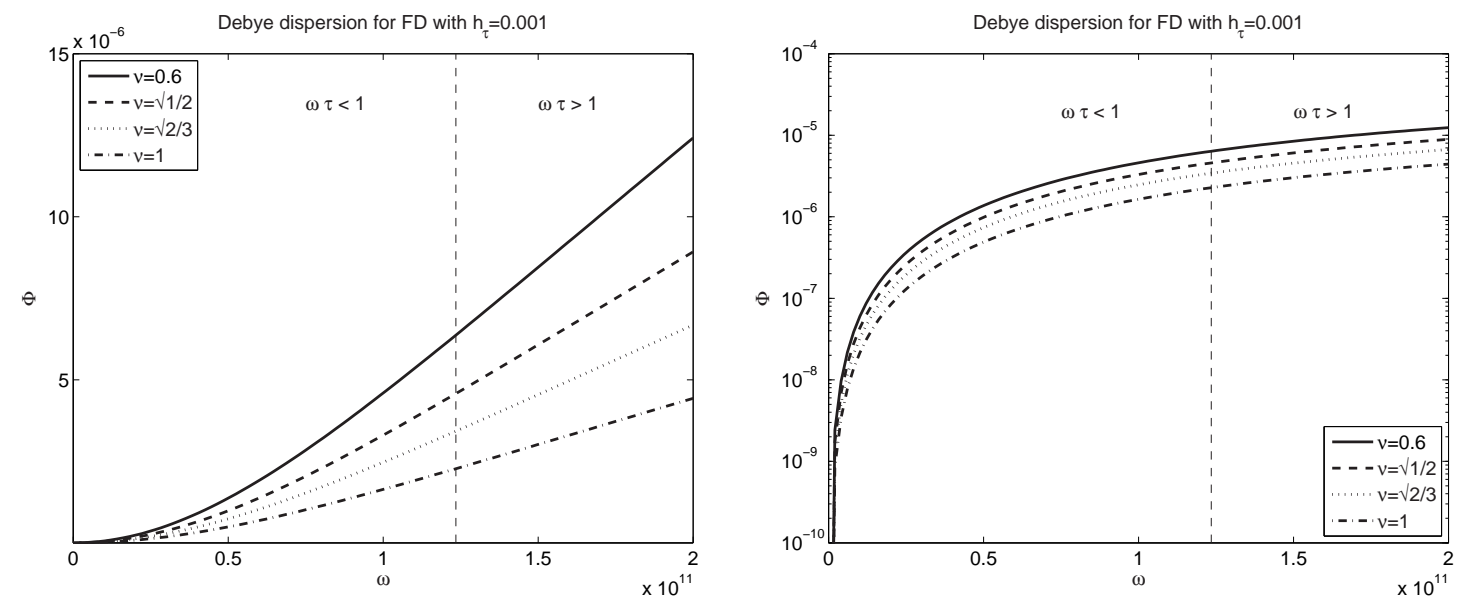

Figure 28: Plot of the phase error $\phi$ versus $\omega$ for the finite difference scheme of the Debye model with $h_{\tau}=0.001$ using $\nu=\{0.6, \sqrt{1 / 2}, \sqrt{2 / 3}, 1\}$. (Right plot is log of left plot.) 

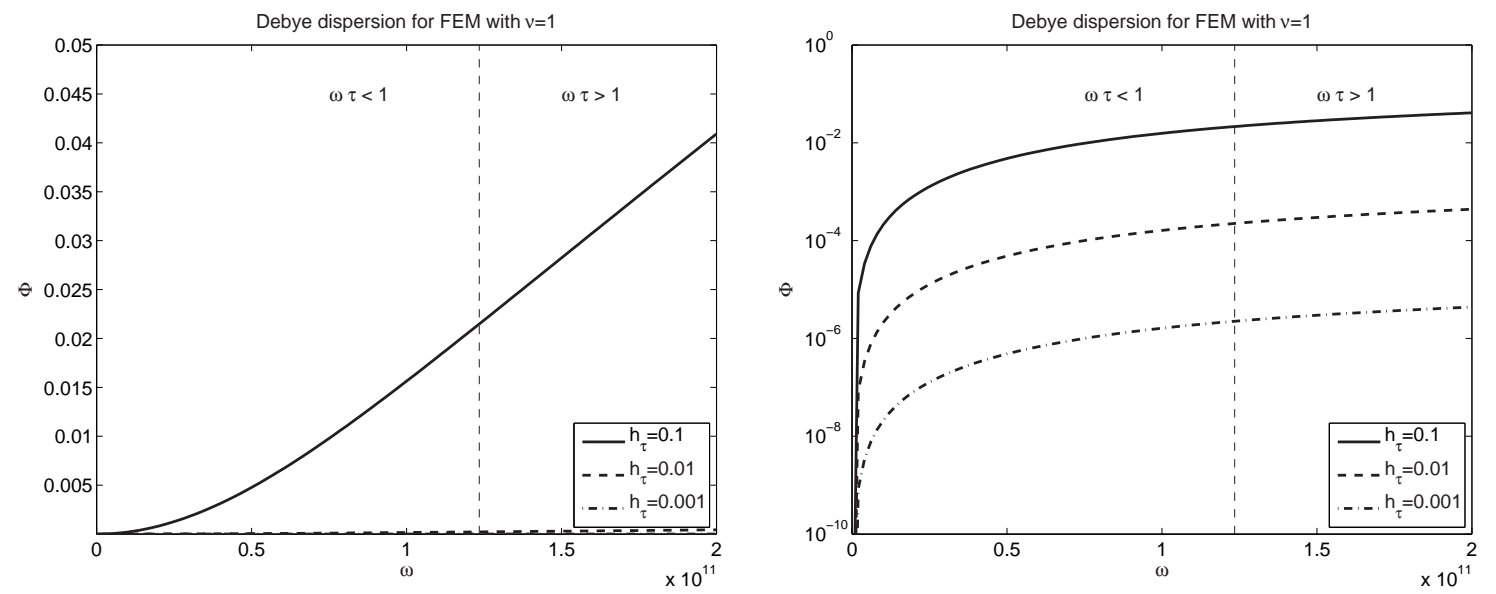

Figure 29: Plot of the phase error $\phi$ versus $\omega$ for the finite element scheme of the Debye model with $\nu=1$ using $h_{\tau}=\{0.1,0.01,0.001\}$. (Right plot is log of left plot.)
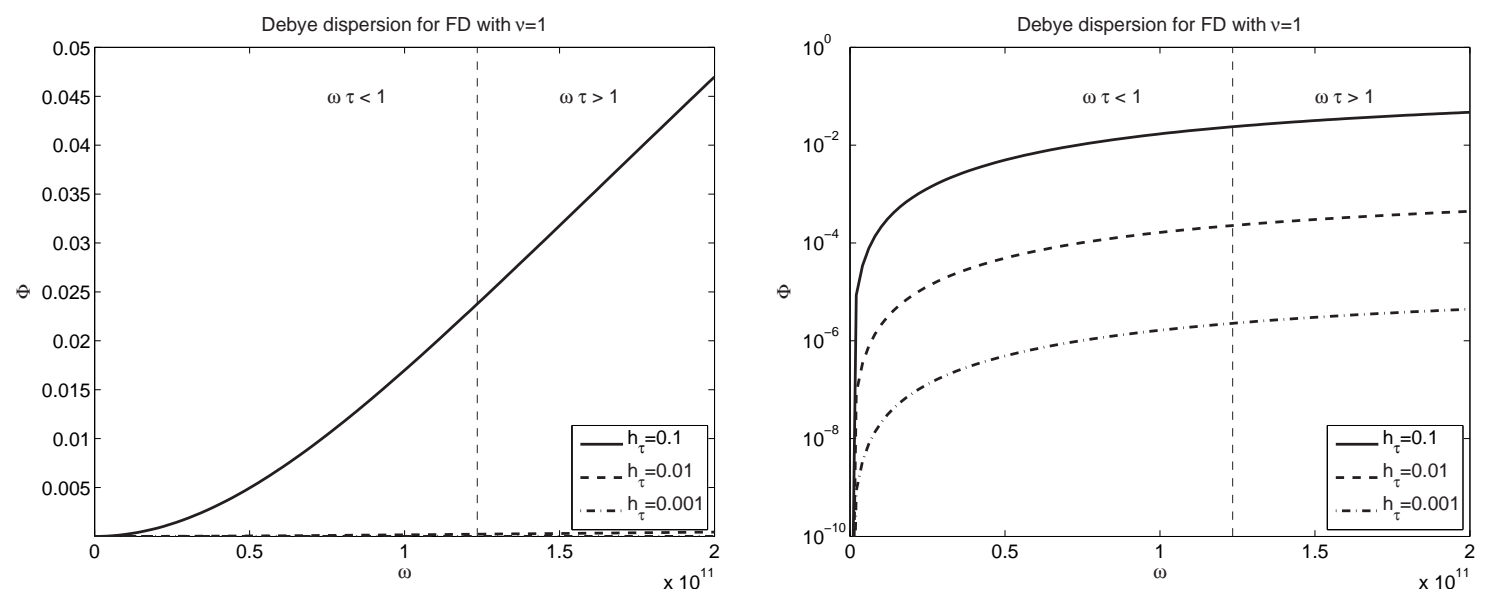

Figure 30: Plot of the phase error $\phi$ versus $\omega$ for the finite difference scheme of the Debye model with $\nu=1$ using $h_{\tau}=\{0.1,0.01,0.001\}$. (Right plot is log of left plot.) 

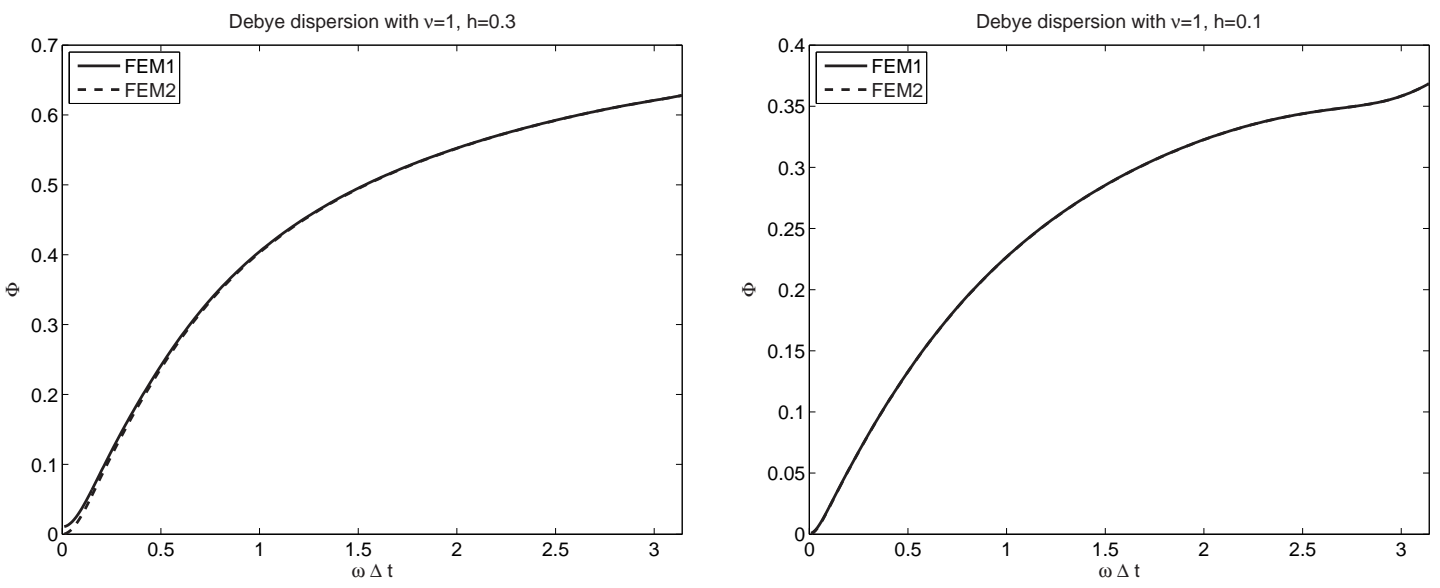

Figure 31: Comparison of the phase error in the two finite element schemes with $\nu=1$ and $h_{\tau}=0.3$ (left) and $h_{\tau}=0.1$ (right). 


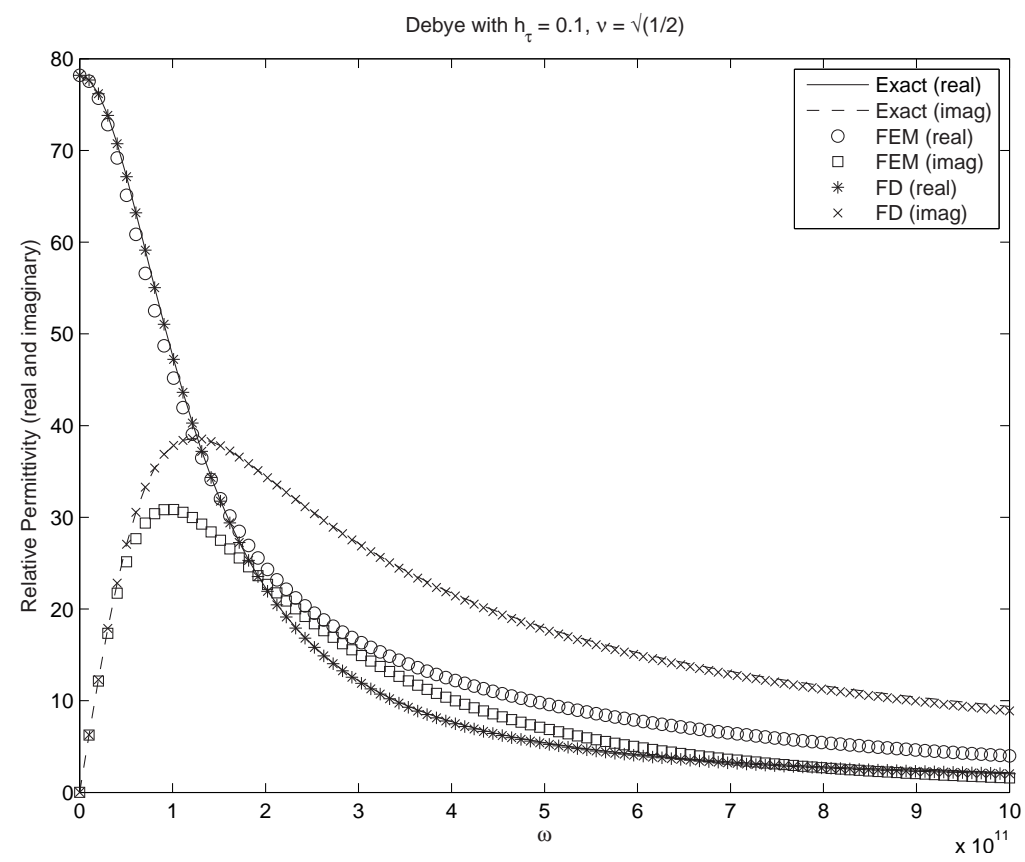

Figure 32: Plots of the real and imaginary parts of the relative complex permittivity for Debye media for the continuous equations, the finite element scheme with $\nu=\sqrt{1 / 2}, h_{\tau}=$ 0.1 and the finite difference scheme with $\nu=1, h_{\tau}=0.1$.

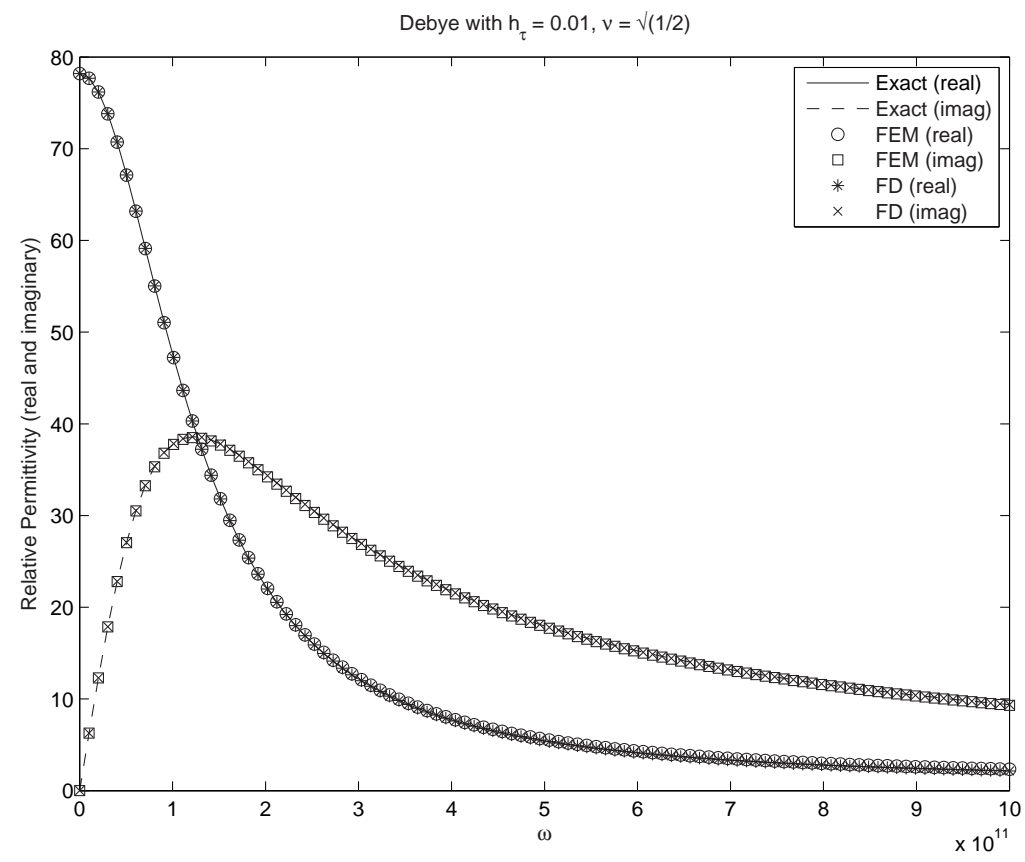

Figure 33: Plots of the real and imaginary parts of the relative complex permittivity for Debye media for the continuous equations, the finite element scheme with $\nu=\sqrt{1 / 2}, h_{\tau}=$ 0.01 and the finite difference scheme with $\nu=1, h_{\tau}=0.01$. 


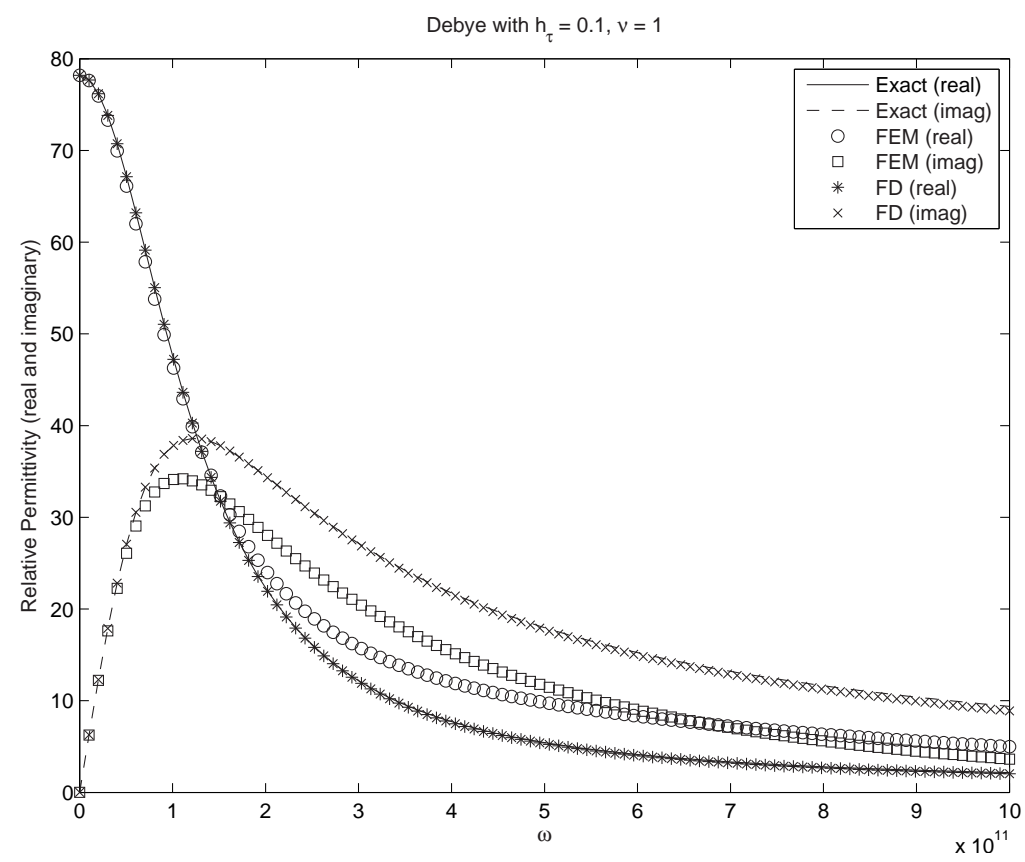

Figure 34: Plots of the real and imaginary parts of the relative complex permittivity for Debye media for the continuous equations, the finite element scheme with $\nu=1, h_{\tau}=0.1$ and the finite difference scheme with $\nu=1, h_{\tau}=0.1$.

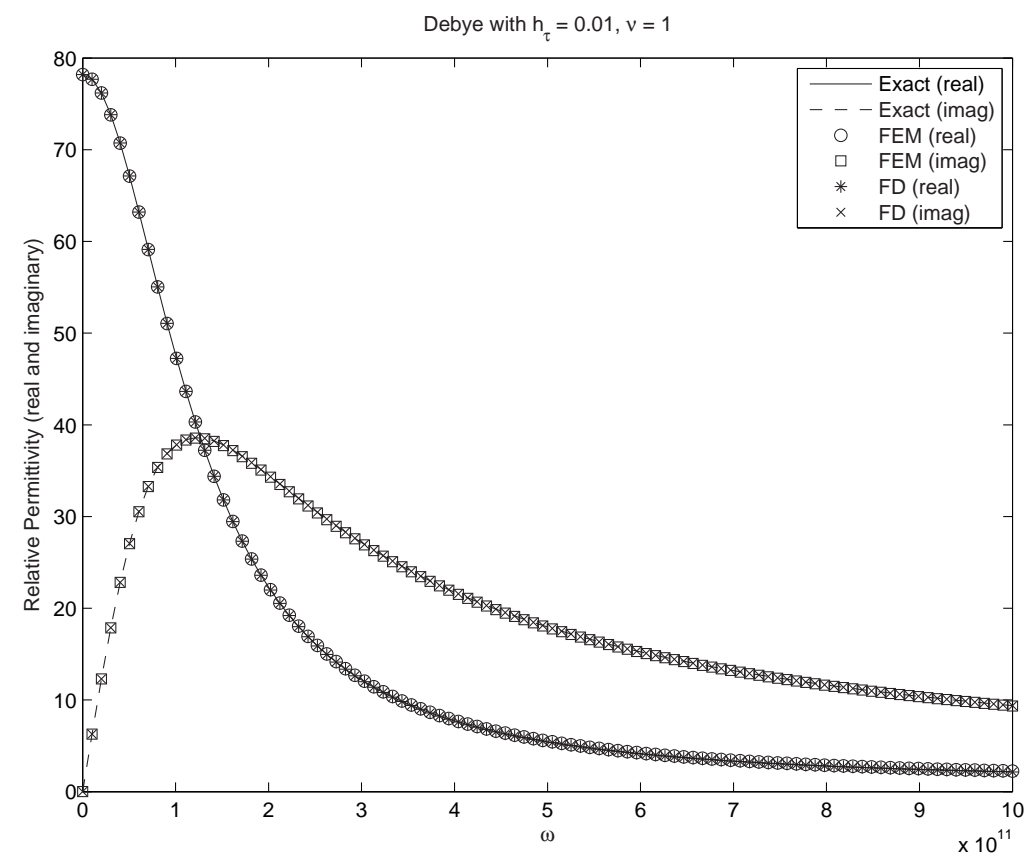

Figure 35: Plots of the real and imaginary parts of the relative complex permittivity for Debye media for the continuous equations, the finite element scheme with $\nu=1, h_{\tau}=0.01$ and the finite difference scheme with $\nu=1, h_{\tau}=0.01$. 


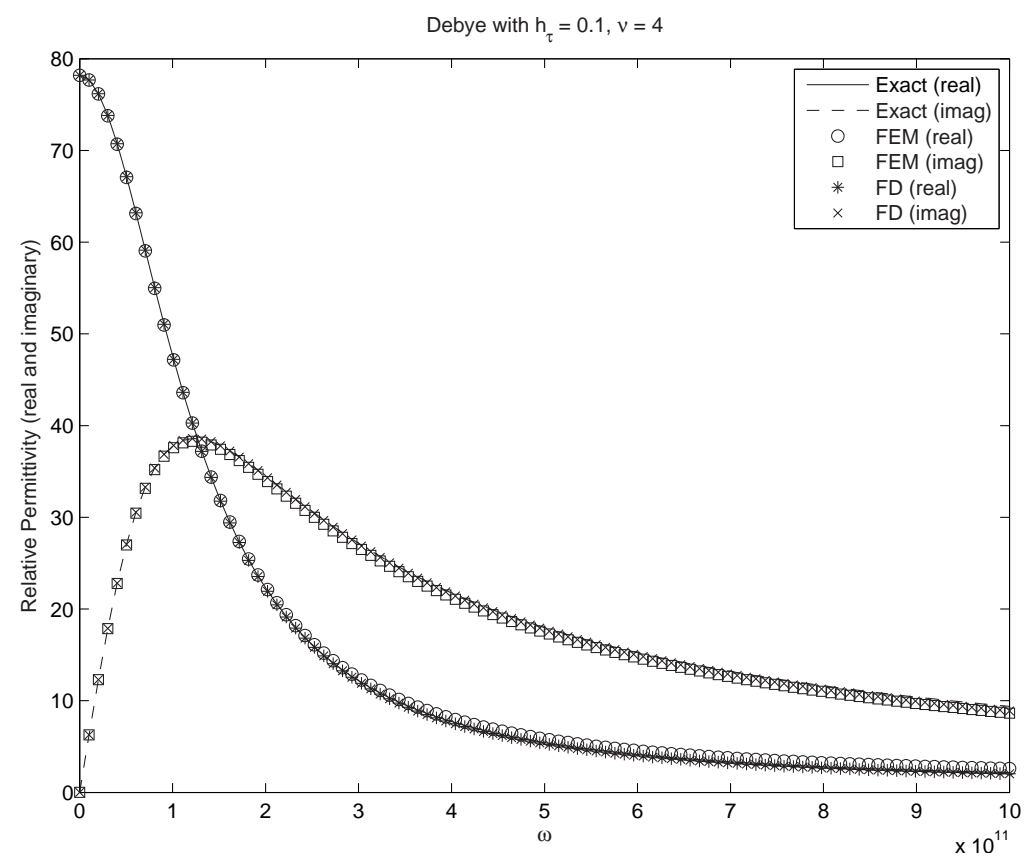

Figure 36: Plots of the real and imaginary parts of the relative complex permittivity for Debye media for the continuous equations, the finite element scheme with $\nu=4, h_{\tau}=0.1$ and the finite difference scheme with $\nu=1, h_{\tau}=0.1$.

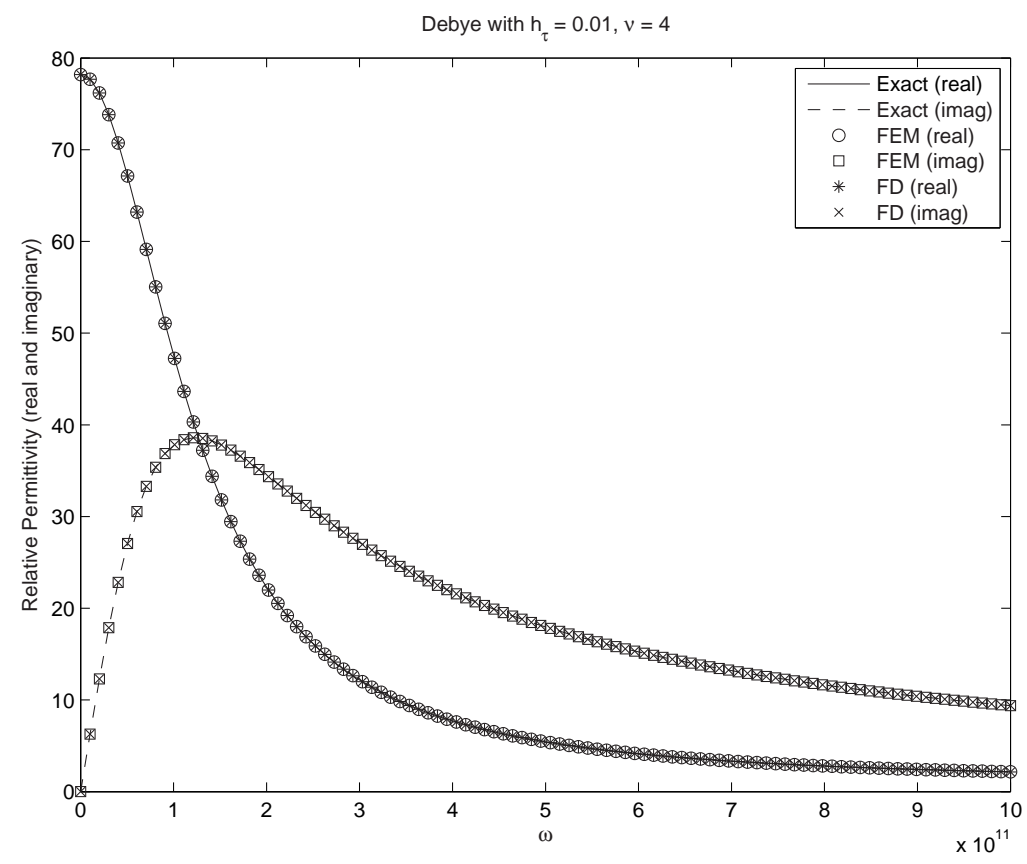

Figure 37: Plots of the real and imaginary parts of the relative complex permittivity for Debye media for the continuous equations, the finite element scheme with $\nu=4, h_{\tau}=0.01$ and the finite difference scheme with $\nu=1, h_{\tau}=0.01$. 

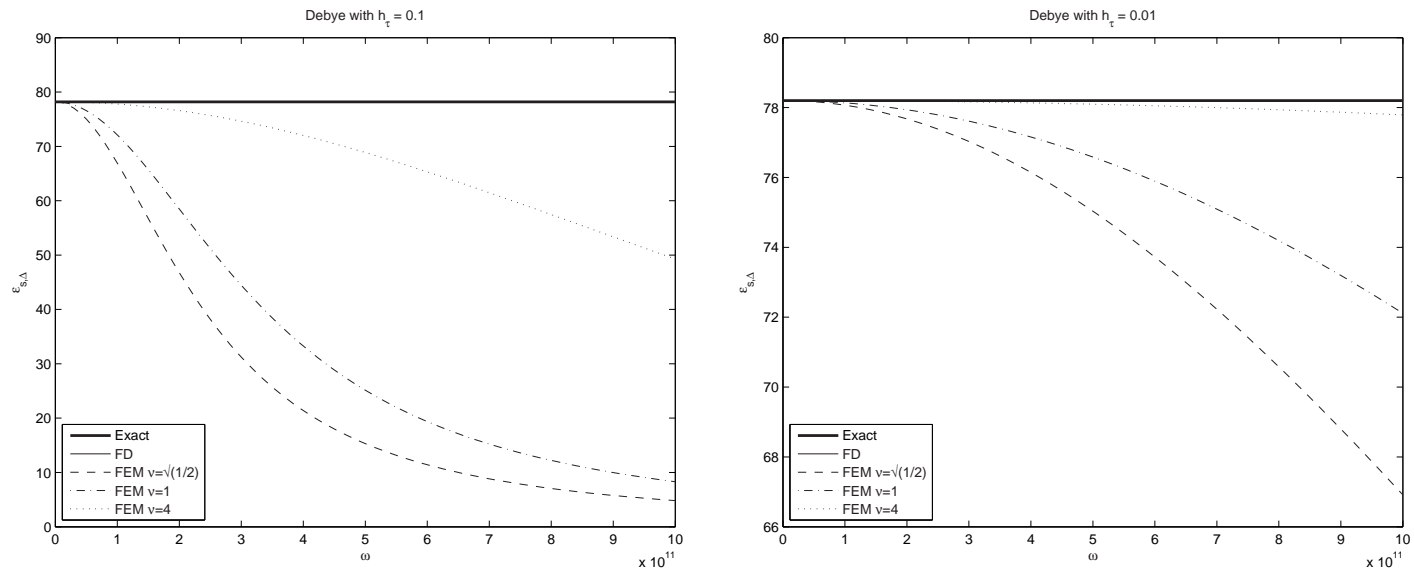

Figure 38: Plots of $\epsilon_{s}$ for Debye media for the continuous equations, the finite element scheme and the finite difference scheme $(\nu=1)$ with $h_{\tau}=0.1$ (left) and $h_{\tau}=0.01$ (right).
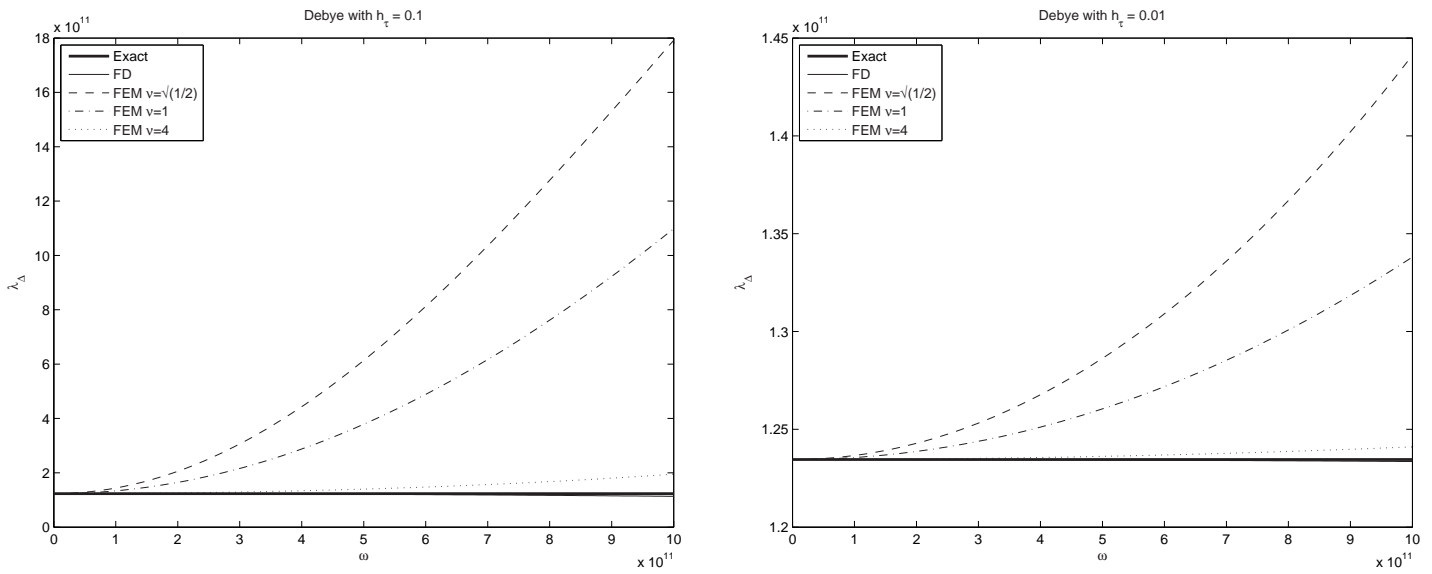

Figure 39: Plots of $\lambda$ for Debye media for the continuous equations, the finite element scheme and the finite difference scheme $(\nu=1)$ with $h_{\tau}=0.1$ (left) and $h_{\tau}=0.01$ (right). 

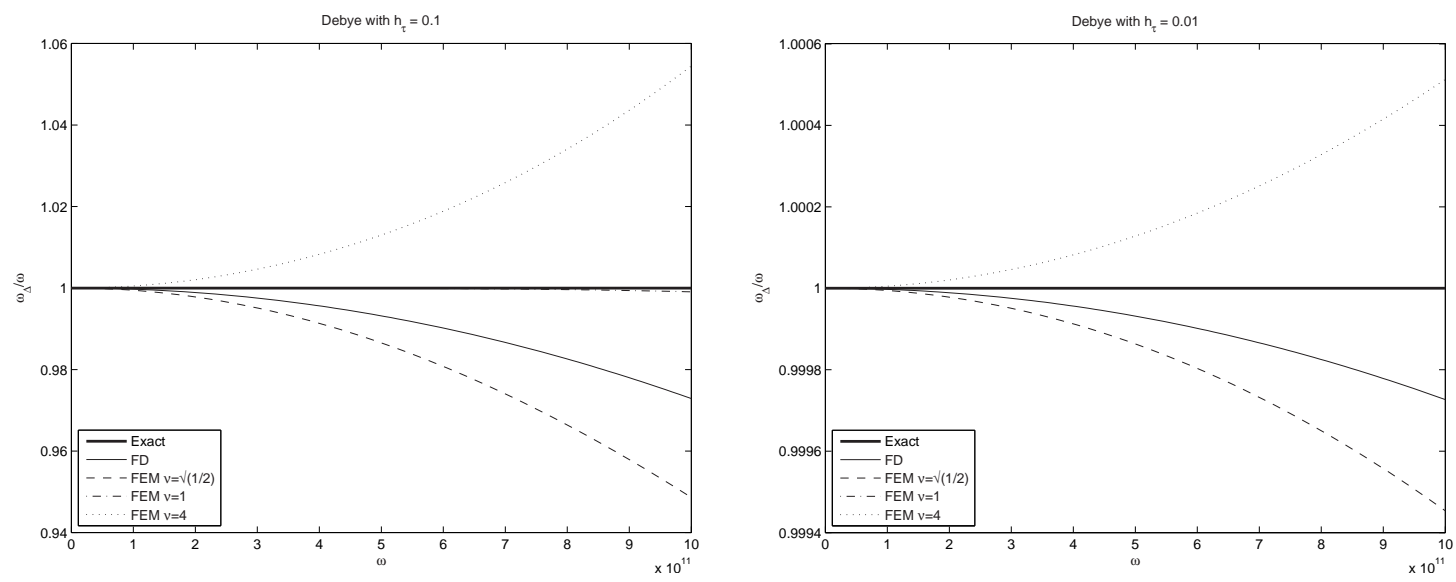

Figure 40: Plots of the frequency $\omega$ for Debye media for the continuous equations, the finite element scheme and the finite difference scheme $(\nu=1)$ with $h_{\tau}=0.1$ (left) and $h_{\tau}=0.01$ (right).

\subsubsection{Lorentz Media}

The dispersion relation for the continuous Lorentz model is given by

$$
k_{\mathrm{EX}}^{\mathrm{L}}(\omega)=\frac{\omega}{c} \sqrt{\epsilon_{r}^{\mathrm{L}}(\omega)} .
$$

where the relative complex permittivity for Lorentz media is given to be

$$
\epsilon_{r}^{\mathrm{L}}(\omega)=\frac{\omega^{2}-\epsilon_{s} \omega_{0}^{2}+i \lambda \omega}{\omega^{2}-\omega_{0}^{2}+i \lambda \omega},
$$

To determine the numerical dispersion relation for the finite element method applied to a Lorentz medium we substitute

$$
X_{j}^{n}=\left[\begin{array}{c}
e_{j}^{n} \\
p_{j}^{n} \\
d_{j}^{n} \\
q_{j}^{n}
\end{array}\right]=\left[\begin{array}{c}
\tilde{e} \\
\tilde{p} \\
\tilde{d} \\
\tilde{q}
\end{array}\right] \mathrm{e}^{i\left(k_{\Delta} j \Delta z-\omega n \Delta t\right)}
$$

into the discrete equations (3.19),(3.20) and (3.21). Again, we asssume that $\epsilon_{\infty}=1$ in the following analysis.

As before we obtain a homogeneous system of the type $\mathcal{A} \tilde{\mathcal{X}}=0$. We then $\operatorname{set} \operatorname{det}(\mathcal{A})=0$ to obtain a relation between the numerical wavenumber $k_{\Delta}$ and the frequency $\omega$. Solving for $k_{\Delta}$ in this relation, and writing in a form comparable to (5.36), we have that the numerical dispersion relation for the FEM scheme applied to Lorentz media is

$$
k_{\Delta}=k_{\mathrm{FE}}^{\mathrm{L}}(\omega)=\frac{2}{\Delta z} \sin ^{-1}\left[\frac{\omega_{\Delta}}{c} \frac{\Delta z}{2} \sqrt{\epsilon_{r, \mathrm{FE}}^{\mathrm{L}}}\right] \text {, }
$$


where the discrete relative complex permittivity is given to be

$$
\epsilon_{r, \mathrm{FE}}^{\mathrm{L}}=\frac{\omega_{\Delta}^{2}-\epsilon_{s, \Delta} \omega_{0, \Delta}^{2}+i \lambda_{\Delta} \omega_{\Delta}}{\omega_{\Delta}^{2}-\omega_{0, \Delta}^{2}+i \lambda_{\Delta} \omega_{\Delta}} .
$$

Here

$$
\begin{aligned}
\epsilon_{s, \Delta} & =\frac{\epsilon_{s}}{\alpha^{2} \beta^{2}} \\
\lambda_{\Delta} & =\lambda \cos (\omega \Delta t / 2) \alpha \\
\omega_{0, \Delta} & =\omega_{0} \cos (\omega \Delta t / 2) \beta \alpha^{2} \\
\omega_{\Delta} & =\omega s_{\omega} \alpha,
\end{aligned}
$$

where $s_{\omega}, \alpha, \beta$ are as defined in equations (5.23)-(5.25). Thus, the FEM scheme misrepresents $\epsilon_{s}, \lambda, \omega_{0}$ and $\omega$ as $\epsilon_{s, \Delta}, \lambda_{\Delta}, \omega_{0, \Delta}$ and $\omega_{\Delta}$ respectively. In particular, note that for the finite element method applied to the Lorentz model, $\lambda_{\Delta}$ does not depend on the continuous model parameter $\epsilon_{s}$, however, $\omega_{0, \Delta}$ does. In the Debye model, $\lambda_{\Delta}$ depended on $\beta^{2}$ whereas for the Lorentz model, $\omega_{0, \Delta}$ depends directly on $\beta$. However, the discrete parameter appears as $\omega_{0, \Delta}^{2}$ in the dispersion relation, so the contribution from $\beta$ is again raised to the second power. This coupled with the fact that the regime of interest is $\omega \Delta t$ small, means that again the effect of $\epsilon_{s}$ on the dispersion caused by the $\omega_{0, \Delta}$ term is likely to be small.

We compare the FEM scheme for Lorentz media with two different finite difference schemes which have been analyzed in [28]. For the scheme in [18], which we will refer to as the JHT scheme, the numerical dispersion relation is given as

$$
k_{\Delta}=k_{\mathrm{JHT}}^{\mathrm{L}}(\omega)=\frac{2}{\Delta z} \sin ^{-1}\left[\frac{\omega}{c} \frac{\Delta z}{2} s_{\omega} \sqrt{\epsilon_{r, \mathrm{JHT}}^{\mathrm{L}}}\right]
$$

where the discrete relative complex permittivity for the JHT scheme is

$$
\epsilon_{r, \mathrm{JHT}}^{\mathrm{L}}=\frac{\omega_{\Delta}^{2}-\epsilon_{s, \Delta} \omega_{0, \Delta}^{2}+i \lambda_{\Delta} \omega_{\Delta}}{\omega_{\Delta}^{2}-\omega_{0, \Delta}^{2}+i \lambda_{\Delta} \omega_{\Delta}} .
$$

Here the discrete representations of the continuous model parameters and the frequency are given as

$$
\begin{aligned}
\epsilon_{s, \Delta} & =\epsilon_{s} \\
\lambda_{\Delta} & =\lambda \frac{\tilde{s}_{\omega}}{s_{\omega}} \\
\omega_{0, \Delta} & =\omega_{0} \sqrt{\cos (\omega \Delta t)} \\
\omega_{\Delta} & =\omega s_{\omega},
\end{aligned}
$$

where $\tilde{s}_{\omega}=\frac{\sin (\omega \Delta t)}{\omega \Delta t}$.

The second finite difference scheme is presented in [19] and will be refered to as the KF scheme. The numerical dispersion relation for this scheme is given by

$$
k_{\mathrm{KF}}^{\mathrm{L}}(\omega)=\frac{2}{\Delta z} \sin ^{-1}\left[\frac{\omega}{c} \frac{\Delta z}{2} s_{\omega} \sqrt{\epsilon_{r, \mathrm{KF}}^{\mathrm{L}}}\right],
$$


where the discrete relative complex permittivity for the KF scheme is

$$
\epsilon_{r, \mathrm{KF}}^{\mathrm{L}}=\frac{\omega_{\Delta}^{2}-\epsilon_{s, \Delta} \omega_{0, \Delta}^{2}+i \lambda_{\Delta} \omega_{\Delta}}{\omega_{\Delta}^{2}-\omega_{0, \Delta}^{2}+i \lambda_{\Delta} \omega_{\Delta}} .
$$

The discrete representations of the continuous model parameters and the frequency are given to be

$$
\begin{aligned}
\epsilon_{s, \Delta} & =\epsilon_{s} \\
\lambda_{\Delta} & =\lambda \cos (\omega \Delta t / 2) \\
\omega_{0, \Delta} & =\omega_{0} \cos (\omega \Delta t / 2) \\
\omega_{\Delta} & =\omega s_{\omega} .
\end{aligned}
$$

We now plot the phase error $\Phi$ as defined in (5.10) for the finite element scheme applied to Lorentz media and we compare it with the phase errors for the JHT and the KF finite difference schemes. The phase error is plotted against values of $\omega \Delta t$ in the range $[0, \pi]$. We note that $\omega \Delta t=2 \pi / N_{\mathrm{ppp}}$, where $N_{\mathrm{ppp}}$ is the number of points per period and is related to the number of points per wavelength $N_{\mathrm{ppw}}$ via

$$
N_{\mathrm{ppw}}=\nu N_{\mathrm{ppp}}
$$

To generate the plots below we have used the following values for the medium parameters:

$$
\begin{aligned}
\epsilon_{\infty} & =1 \\
\epsilon_{s} & =2.25 \\
\tau & =1.786 \times 10^{-16} \mathrm{sec} \\
\omega_{0} & =4 \times 10^{16} \mathrm{rad} / \mathrm{sec} .
\end{aligned}
$$

Figures 41-43 plot the phase error $\Phi$ versus $\omega \Delta t$ for the FEM scheme applied to the Lorentz model, with various values of $h_{0}$ and $\nu$. Figures $44-49$ plot the same for the finite difference methods. Figures 50-52 plot the phase error $\Phi$ versus $\omega$ for the FEM scheme applied to the Lorentz model, with various values of $h_{0}$ and $\nu$. Figures $53-58$ plot the same for the finite difference methods. Note that the dispersion for the finite element method reduces as $\nu$ goes to 1 for all values of $h_{0}$.

Figure 59 shows the effect of varying $h_{\tau}$ on the finite element scheme for $\nu=1$. Figures 60 and 61 demonstrate this effect for the JHT and KF finite difference methods, respectively.

In Figures 62-67 we plot the real and imaginary parts of the relative complex permittivity for Lorentz media for the continuous equations (exact values), the finite element scheme with $\nu=\{\sqrt{1 / 2}, 1,4\}, h_{0}=\{0.1,0.01\}$, and finally the finite difference scheme with $\nu=1$ fixed and $h_{0}=\{0.1,0.01\}$. For $h_{0}=0.1$, as $\nu$ is increased, the discrete permittivites of the finite element scheme approach those of the finite difference method. However, for $\nu=1$ it seems that the finite element approximation is actually better. For $h_{0}=0.01$, the agreement of the discrete permittivities with the exact values is better than with $h_{0}=0.1$, for each value of $\nu$. For $\nu=1$ we see the best agreement of the discrete real and imaginary 

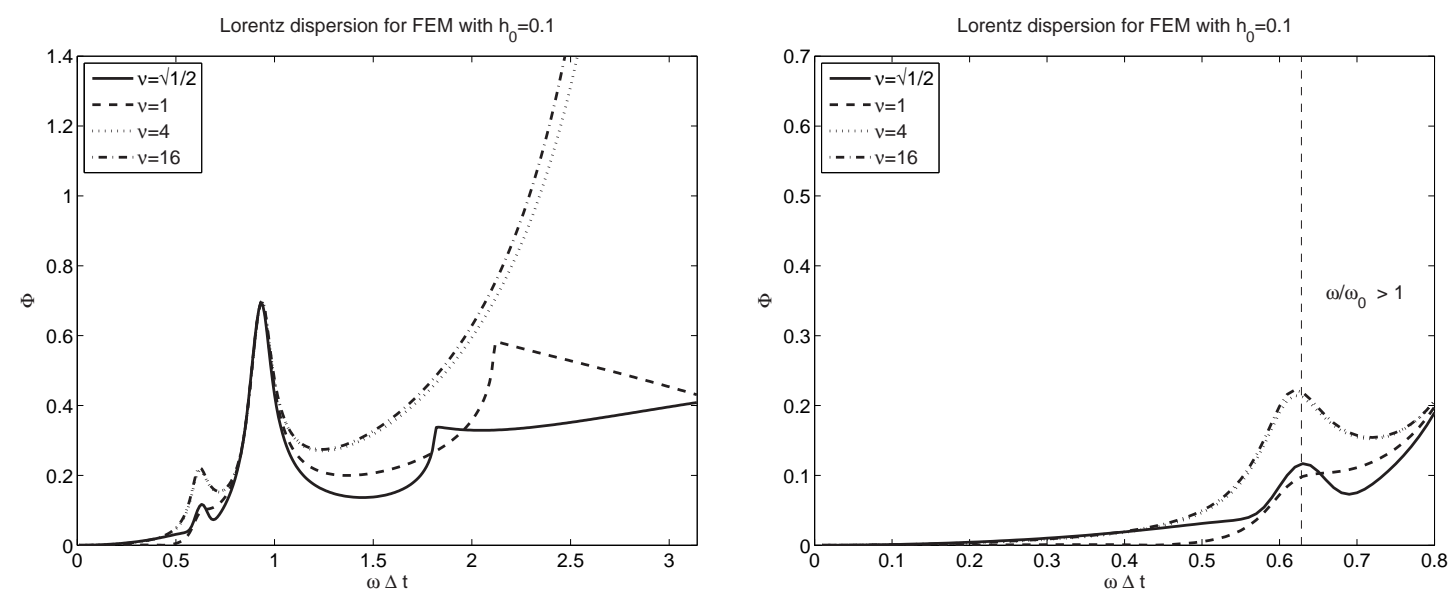

Figure 41: Plot of the phase error $\phi$ versus $\omega \Delta t$ for the finite element scheme of the Lorentz model with $\nu=\{1, \sqrt{2 / 3}, \sqrt{1 / 2}, .6\}$ using $h_{0}=0.1$. (Right plot is zoom of left plot.)
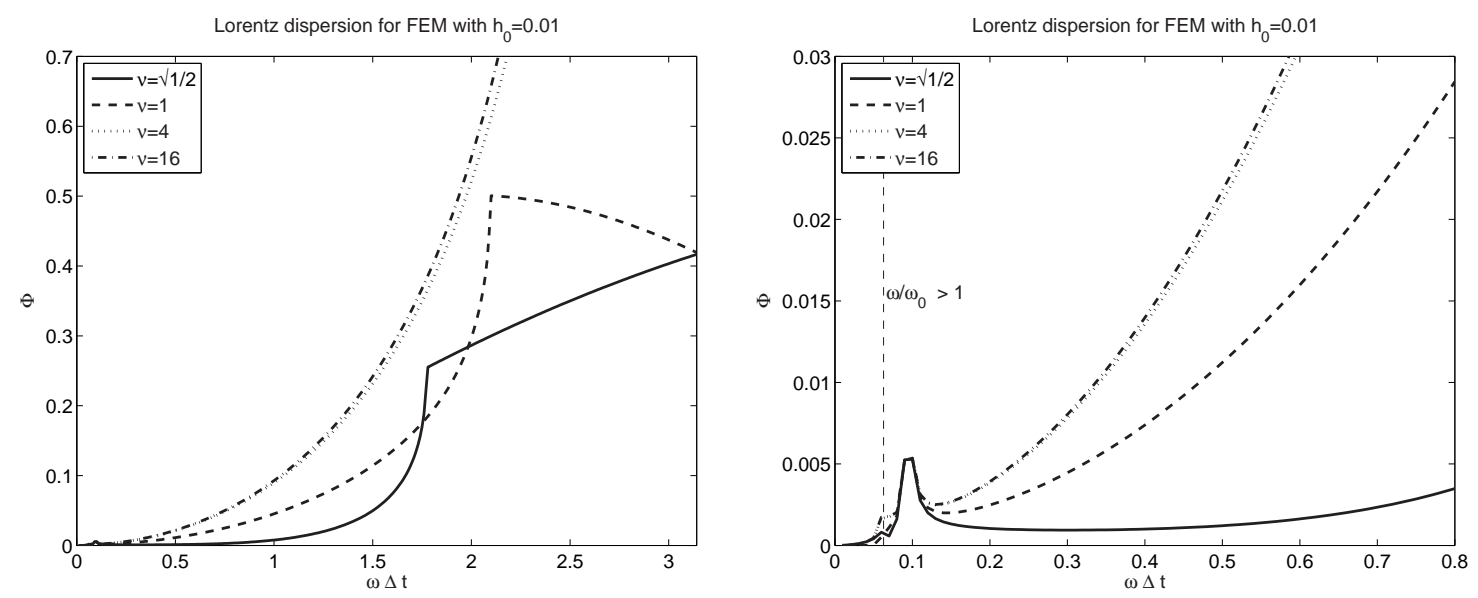

Figure 42: Plot of the phase error $\phi$ versus $\omega \Delta t$ for the finite element scheme of the Lorentz model with $\nu=\{1, \sqrt{2 / 3}, \sqrt{1 / 2}, .6\}$ using $h_{0}=0.01$. (Right plot is zoom of left plot.)

permittivities with the exact values, regardless of $h_{0}$. This is not seen in the plots of the discrete values of the parameters $\lambda, \epsilon_{s}$ and $\omega_{0}$ in Figures 69, 68 and 70, respectively. In these figures we see that the discrete parameters in the finite element scheme have better agreement with the exact values as $\nu$ is increased, even beyond 1 . However the discrete value of the frequency $\omega$, Figure 71 , seems to have a better agreement with the exact value when $\nu=1$. As the discrete complex permittivity matches the exact more closely when $\nu=1$, we conclude that for the Lorentz model, the value of $\nu$ that correctly represents $\omega$ will sufficiently model the complex permittivity. For the finite difference scheme, the discrete parameters do not depend on the value of $\nu$. However, as with the finite element method, if $h_{0}$ is decreased they agree better with the exact values. 

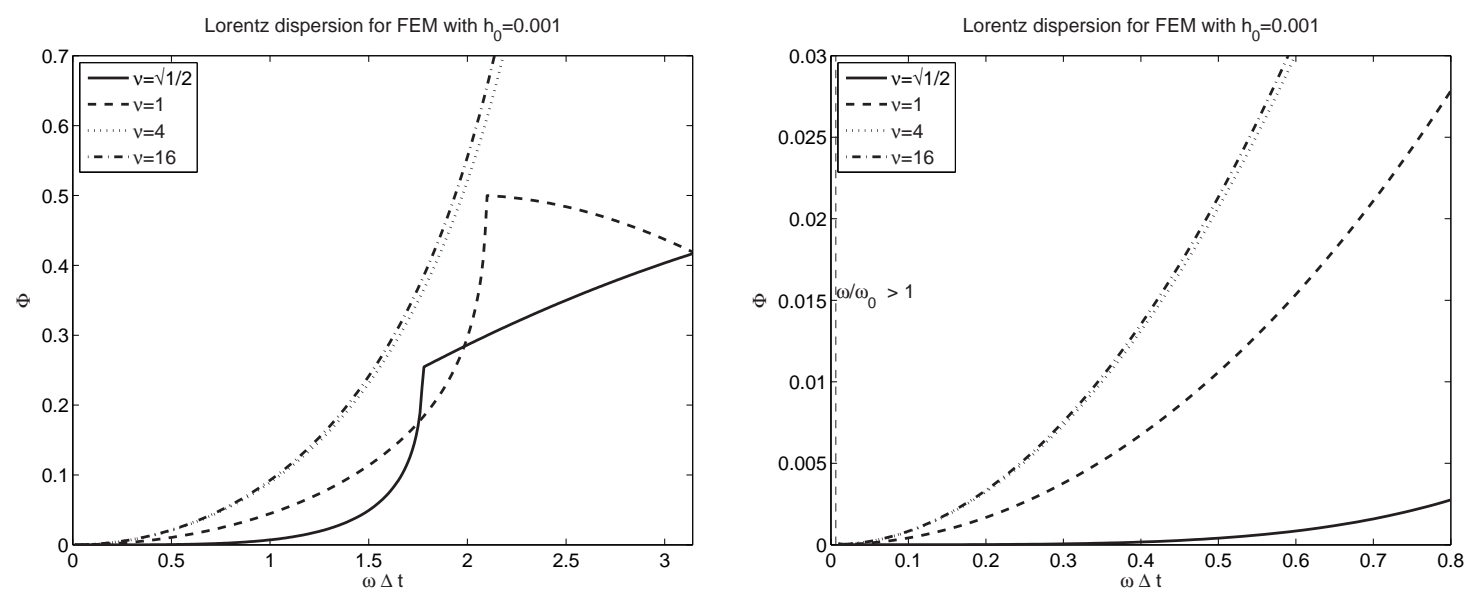

Figure 43: Plot of the phase error $\phi$ versus $\omega \Delta t$ for the finite element scheme of the Lorentz model with $\nu=\{1, \sqrt{2 / 3}, \sqrt{1 / 2}, .6\}$ using $h_{0}=0.001$. (Right plot is zoom of left plot.)
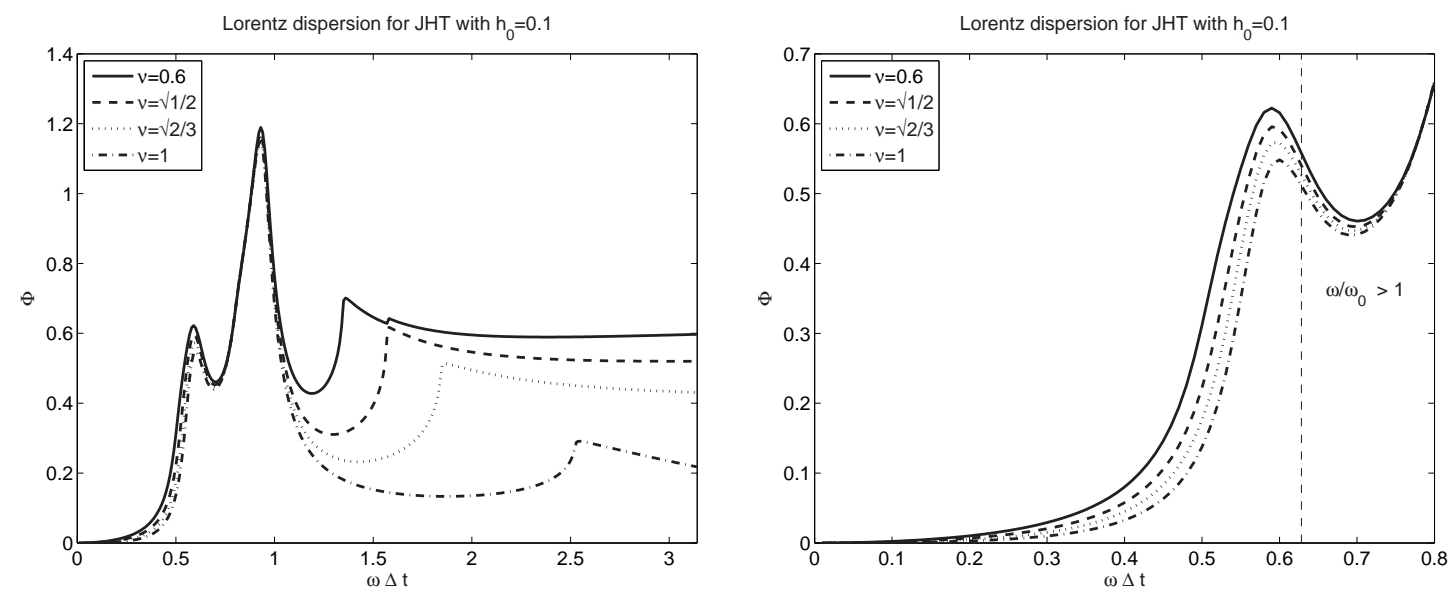

Figure 44: Plot of the phase error $\phi$ versus $\omega \Delta t$ for the JHT finite difference scheme of the Lorentz model with $\nu=\{1, \sqrt{2 / 3}, \sqrt{1 / 2}, .6\}$ using $h_{0}=0.1$. (Right plot is zoom of left plot.) 

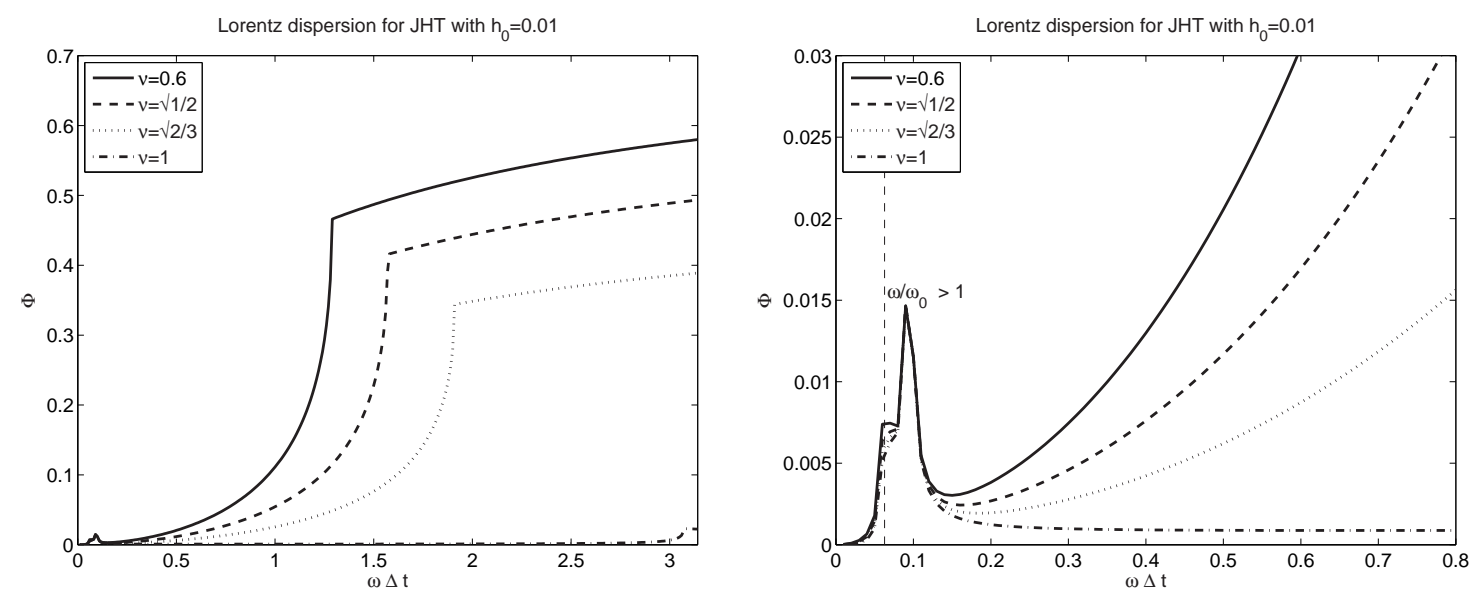

Figure 45: Plot of the phase error $\phi$ versus $\omega \Delta t$ for the JHT finite difference scheme of the Lorentz model with $\nu=\{1, \sqrt{2 / 3}, \sqrt{1 / 2}, .6\}$ using $h_{0}=0.01$. (Right plot is zoom of left plot.)
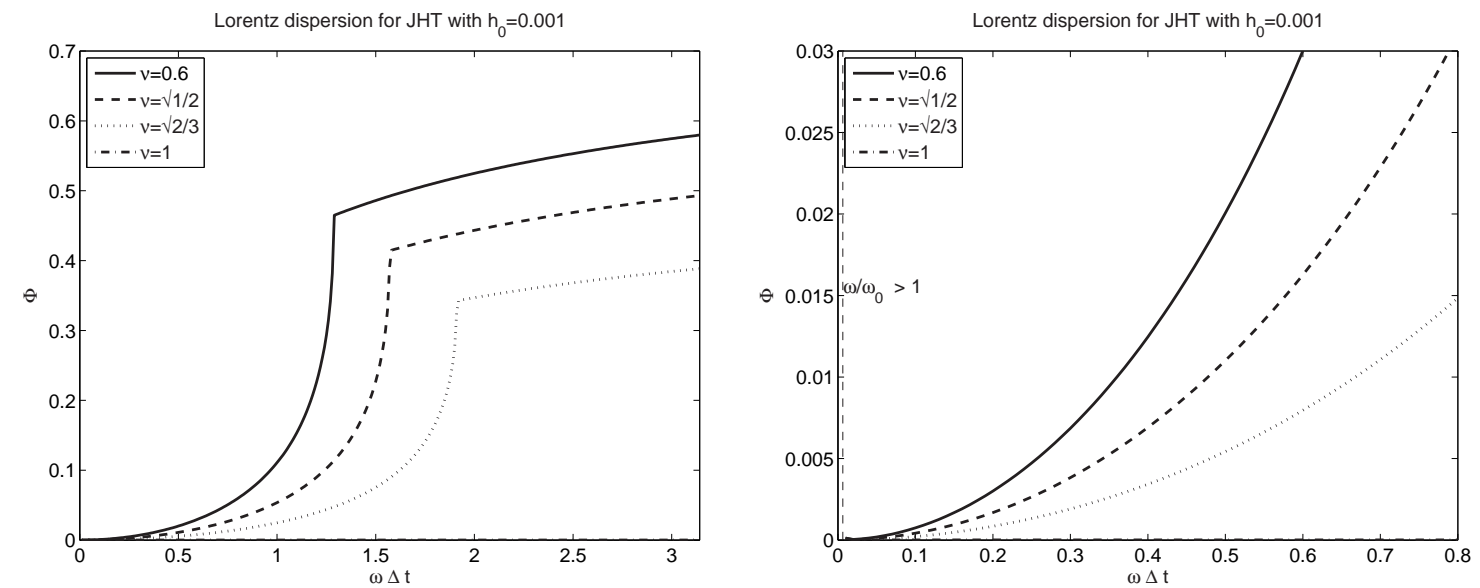

Figure 46: Plot of the phase error $\phi$ versus $\omega \Delta t$ for the JHT finite difference scheme of the Lorentz model with $\nu=\{1, \sqrt{2 / 3}, \sqrt{1 / 2}, .6\}$ using $h_{0}=0.001$. (Right plot is zoom of left plot.) 

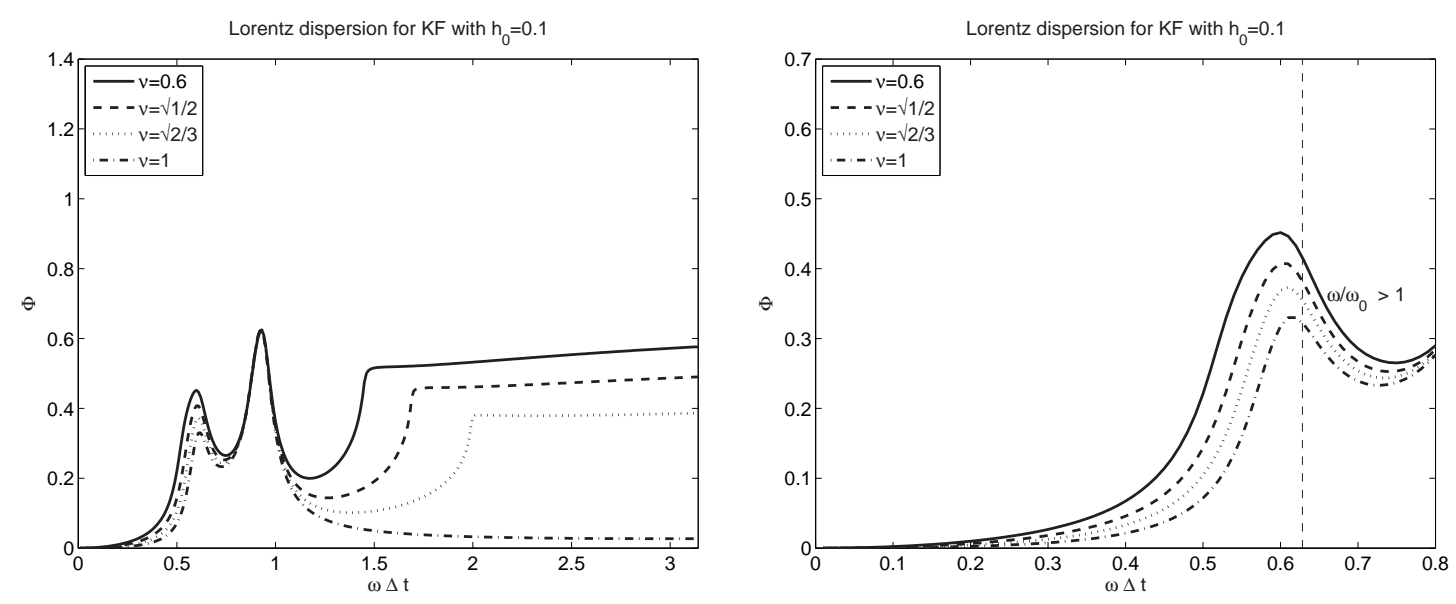

Figure 47: Plot of the phase error $\phi$ versus $\omega \Delta t$ for the KF finite difference scheme of the Lorentz model with $\nu=\{1, \sqrt{2 / 3}, \sqrt{1 / 2}, .6\}$ using $h_{0}=0.1$. (Right plot is zoom of left plot.)
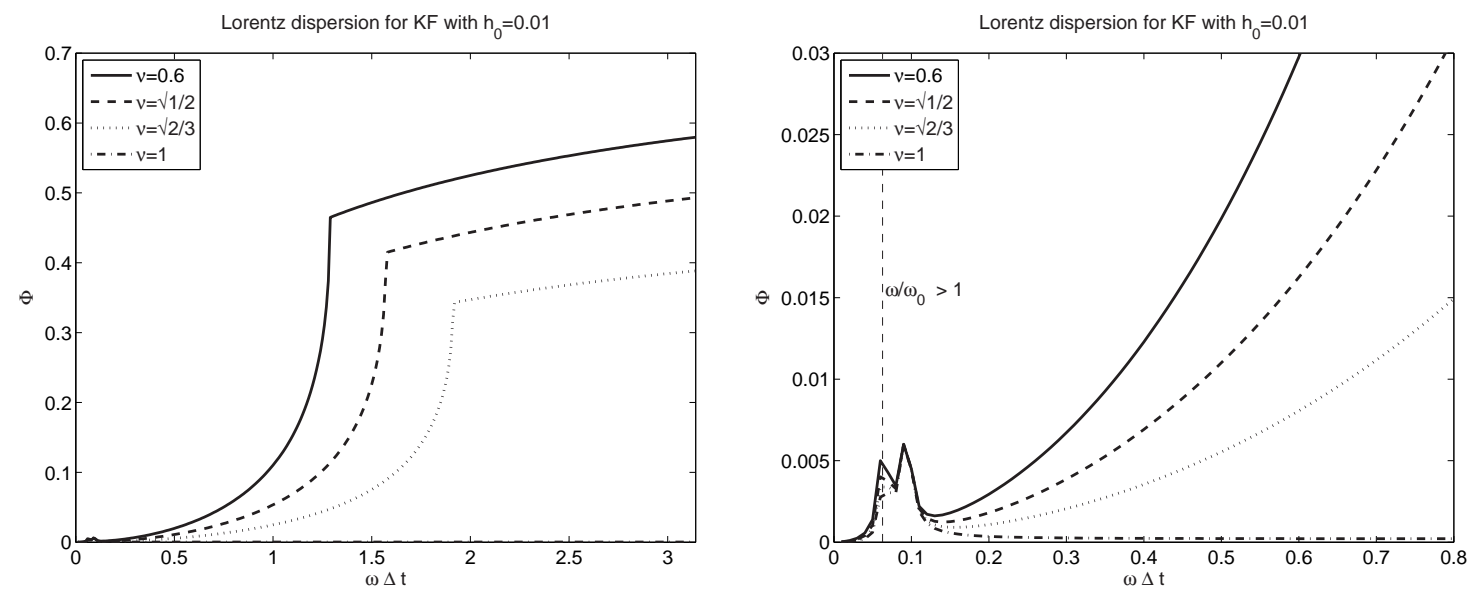

Figure 48: Plot of the phase error $\phi$ versus $\omega \Delta t$ for the KF finite difference scheme of the Lorentz model with $\nu=\{1, \sqrt{2 / 3}, \sqrt{1 / 2}, .6\}$ using $h_{0}=0.01$. (Right plot is zoom of left plot.) 

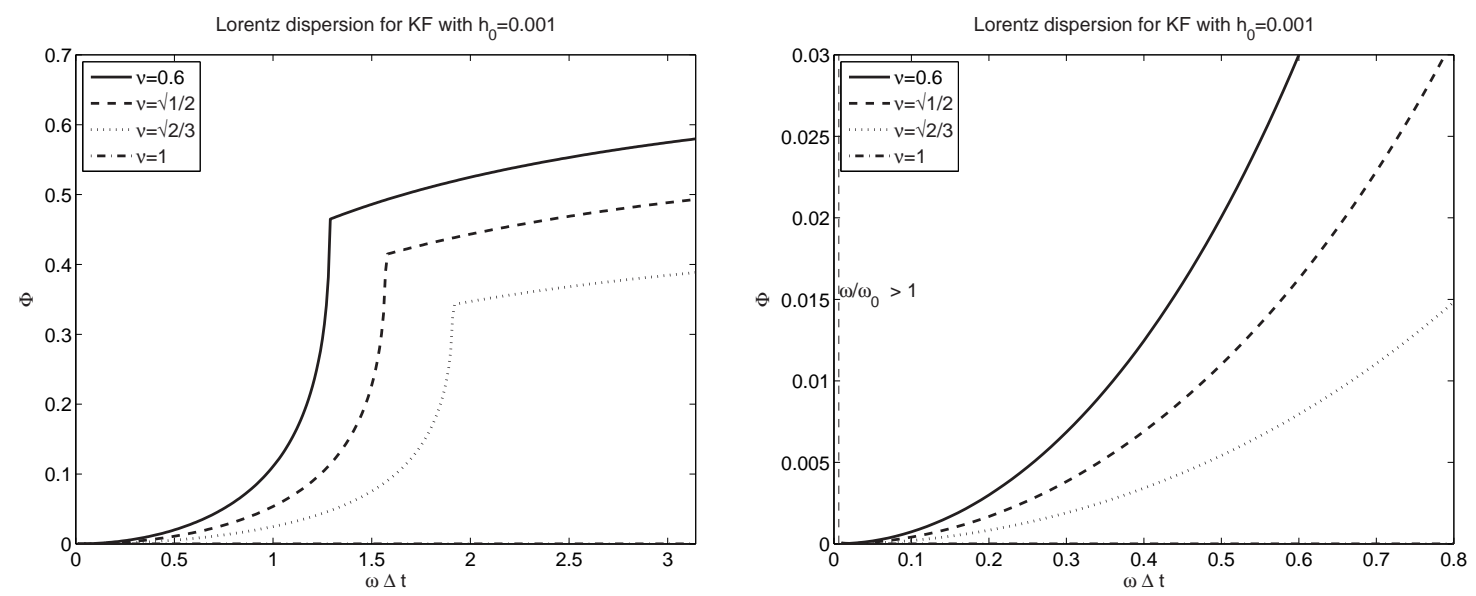

Figure 49: Plot of the phase error $\phi$ versus $\omega \Delta t$ for the KF finite difference scheme of the Lorentz model with $\nu=\{1, \sqrt{2 / 3}, \sqrt{1 / 2}, .6\}$ using $h_{0}=0.001$. (Right plot is zoom of left plot.) 

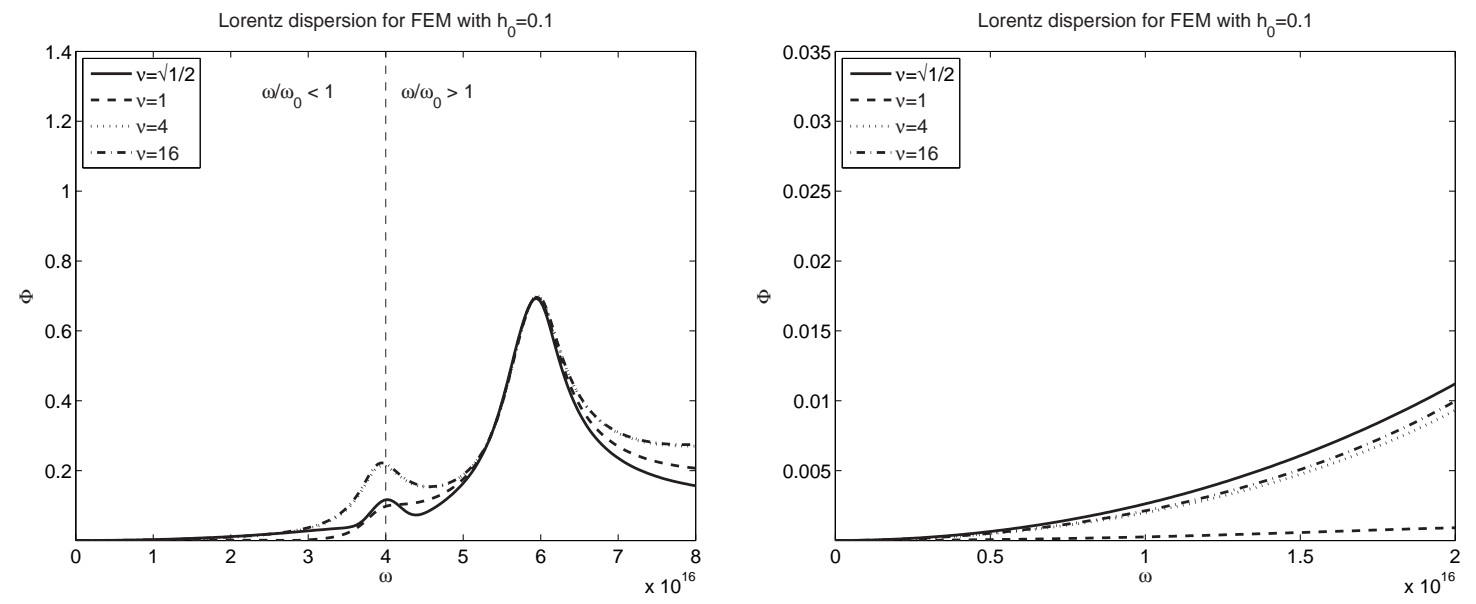

Figure 50: Plot of the phase error $\phi$ versus $\omega$ for the finite element scheme of the Lorentz model with $\nu=\{1, \sqrt{2 / 3}, \sqrt{1 / 2}, .6\}$ using $h_{0}=0.1$. (Right plot is zoom of left plot.)
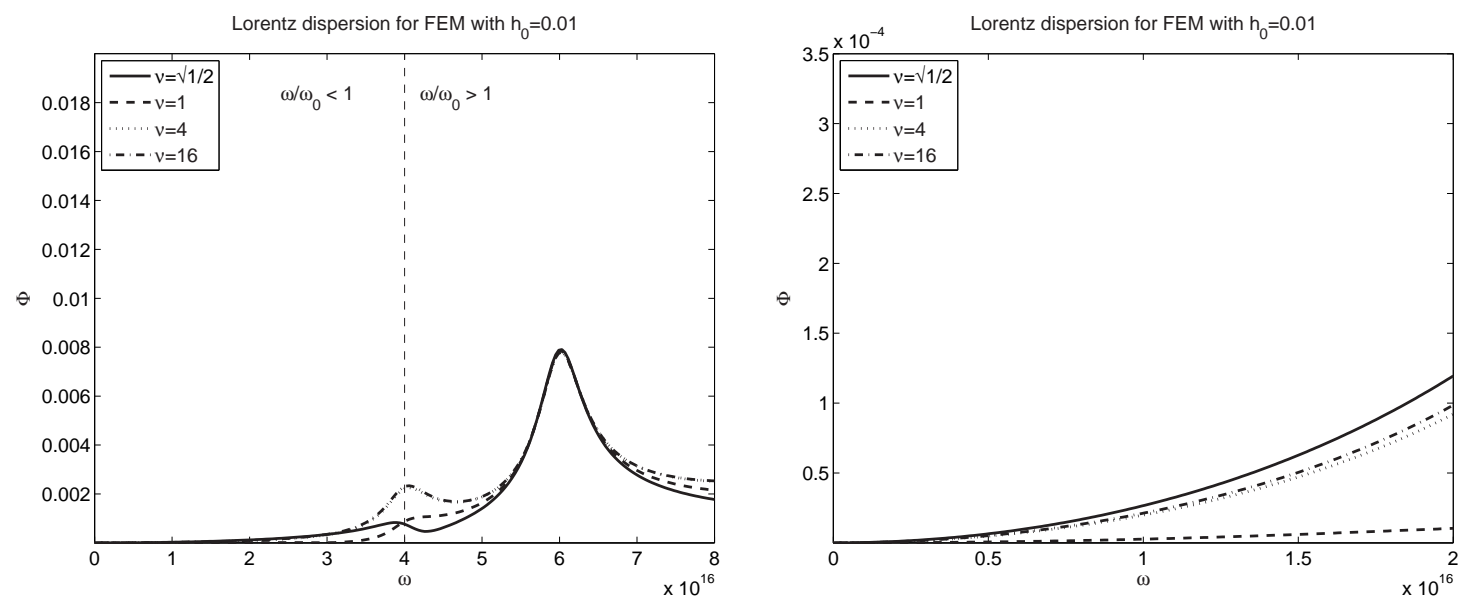

Figure 51: Plot of the phase error $\phi$ versus $\omega$ for the finite element scheme of the Lorentz model with $\nu=\{1, \sqrt{2 / 3}, \sqrt{1 / 2}, .6\}$ using $h_{0}=0.01$. (Right plot is zoom of left plot.) 

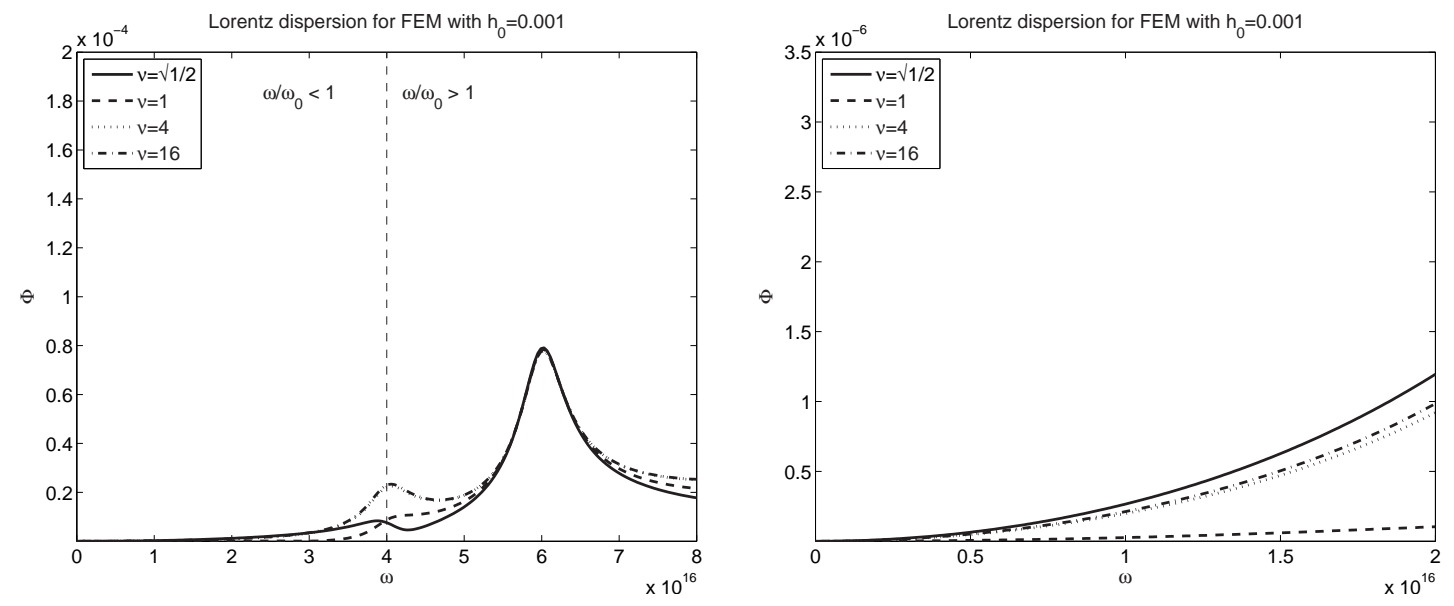

Figure 52: Plot of the phase error $\phi$ versus $\omega$ for the finite element scheme of the Lorentz model with $\nu=\{1, \sqrt{2 / 3}, \sqrt{1 / 2}, .6\}$ using $h_{0}=0.001$. (Right plot is zoom of left plot.)
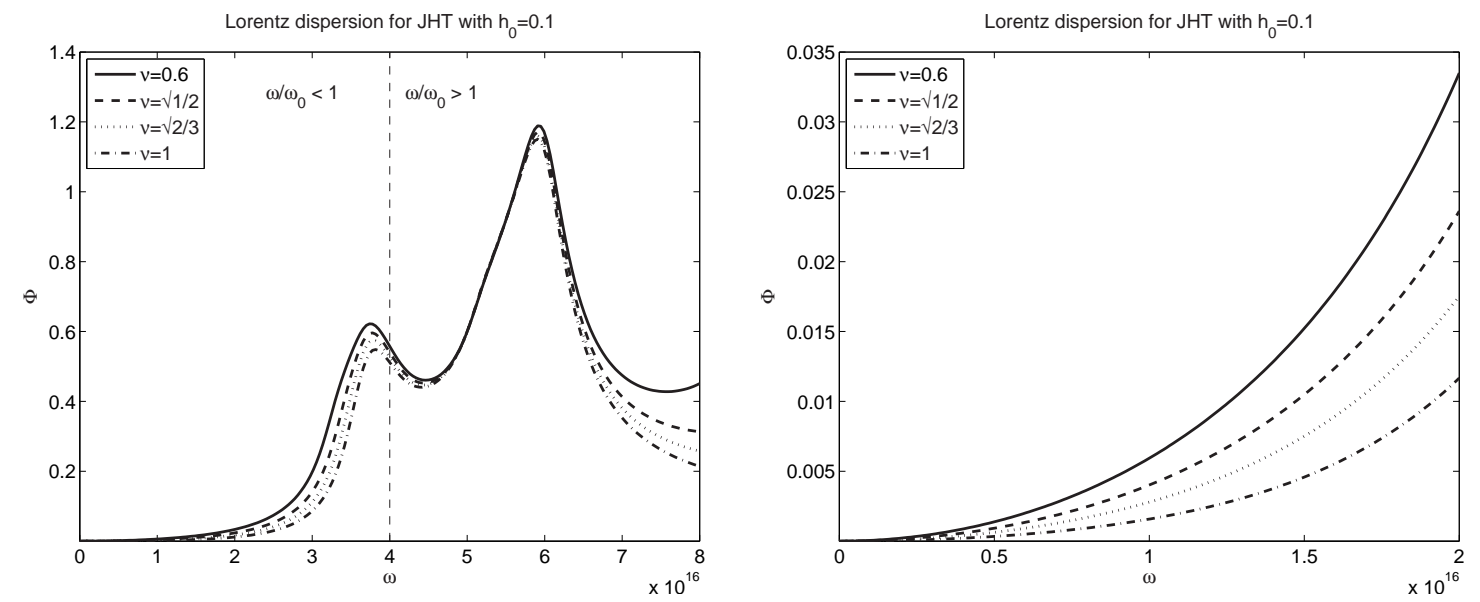

Figure 53: Plot of the phase error $\phi$ versus $\omega$ for the JHT finite difference scheme of the Lorentz model with $\nu=\{1, \sqrt{2 / 3}, \sqrt{1 / 2}, .6\}$ using $h_{0}=0.1$. (Right plot is zoom of left plot.) 

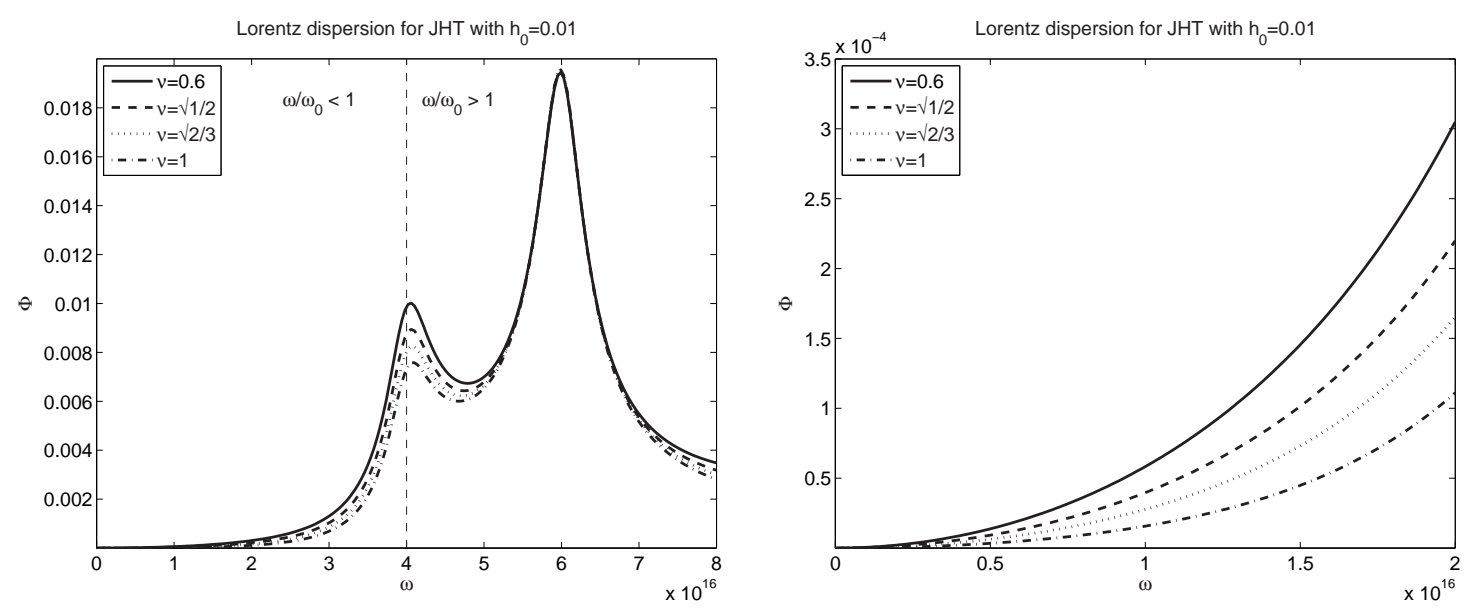

Figure 54: Plot of the phase error $\phi$ versus $\omega$ for the JHT finite difference scheme of the Lorentz model with $\nu=\{1, \sqrt{2 / 3}, \sqrt{1 / 2}, .6\}$ using $h_{0}=0.01$. (Right plot is zoom of left plot.)
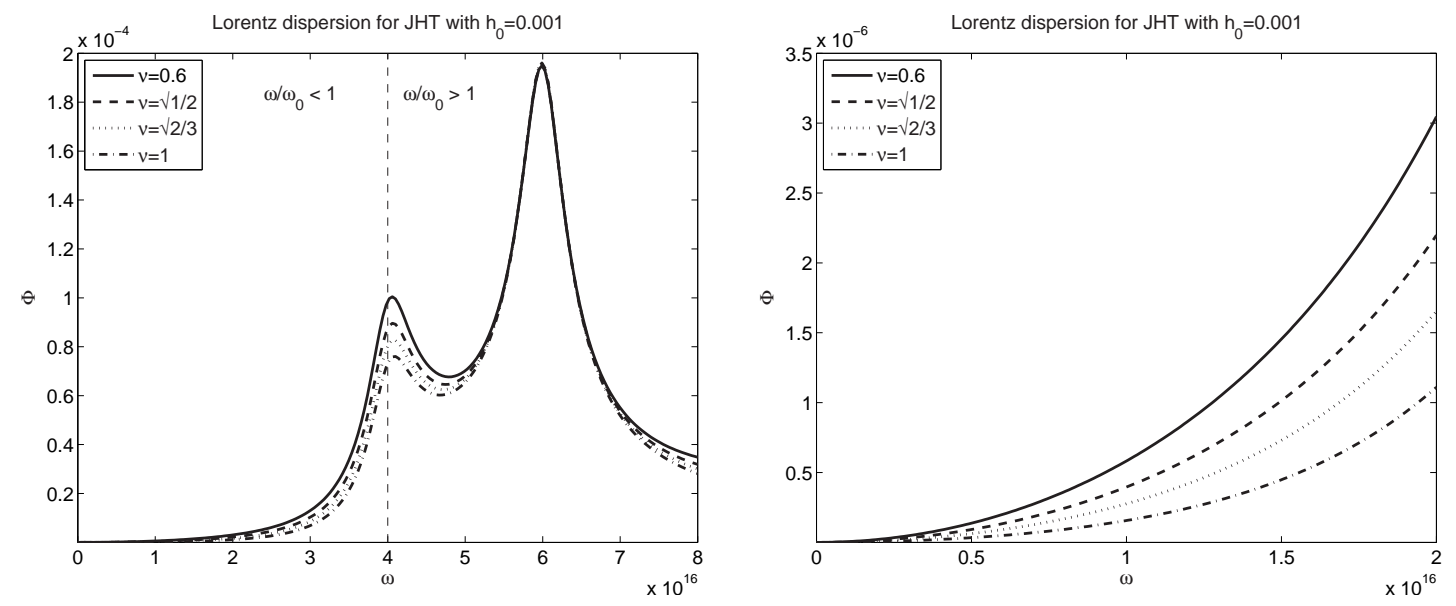

Figure 55: Plot of the phase error $\phi$ versus $\omega$ for the JHT finite difference scheme of the Lorentz model with $\nu=\{1, \sqrt{2 / 3}, \sqrt{1 / 2}, .6\}$ using $h_{0}=0.001$. (Right plot is zoom of left plot.) 

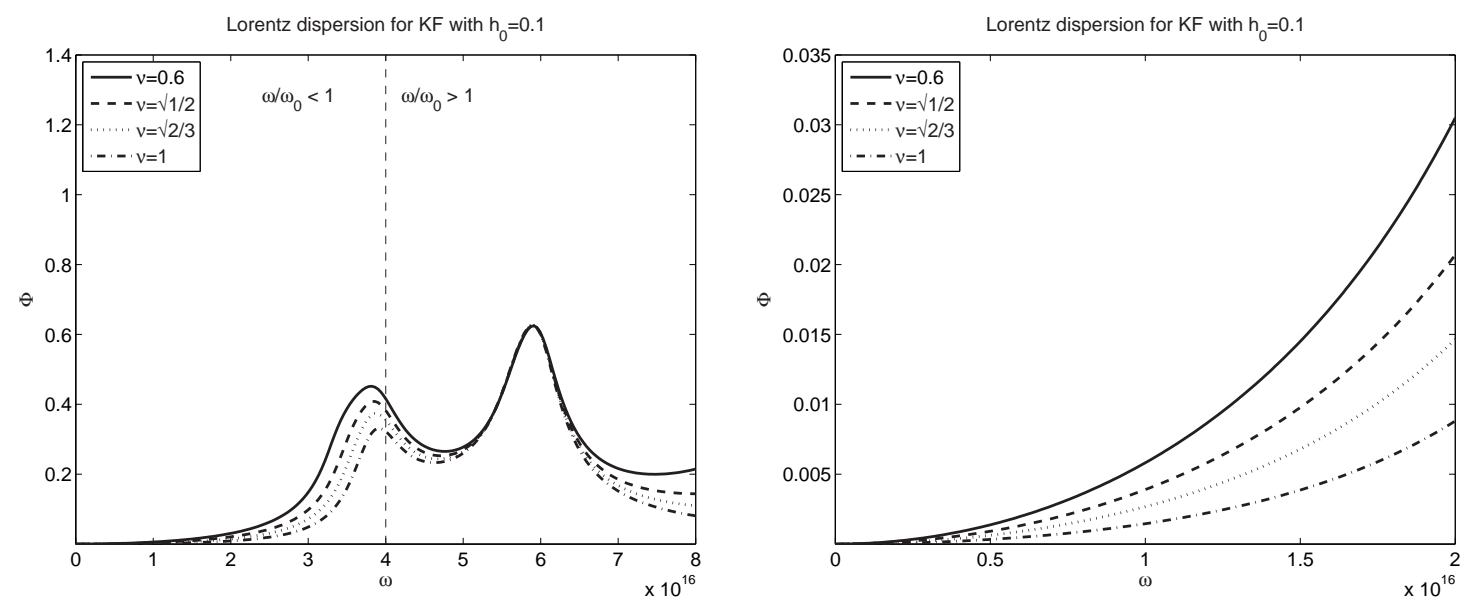

Figure 56: Plot of the phase error $\phi$ versus $\omega$ for the KF finite difference scheme of the Lorentz model with $\nu=\{1, \sqrt{2 / 3}, \sqrt{1 / 2}, .6\}$ using $h_{0}=0.1$. (Right plot is zoom of left plot.)
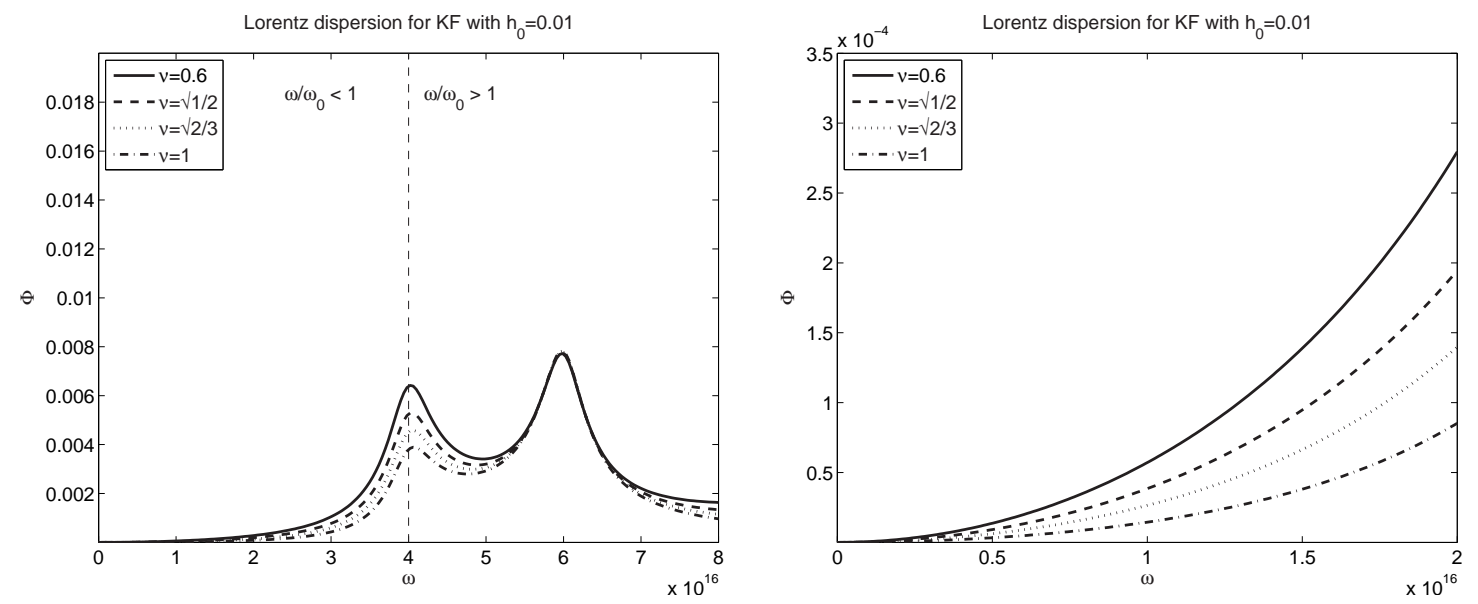

Figure 57: Plot of the phase error $\phi$ versus $\omega$ for the KF finite difference scheme of the Lorentz model with $\nu=\{1, \sqrt{2 / 3}, \sqrt{1 / 2}, .6\}$ using $h_{0}=0.01$. (Right plot is zoom of left plot.) 

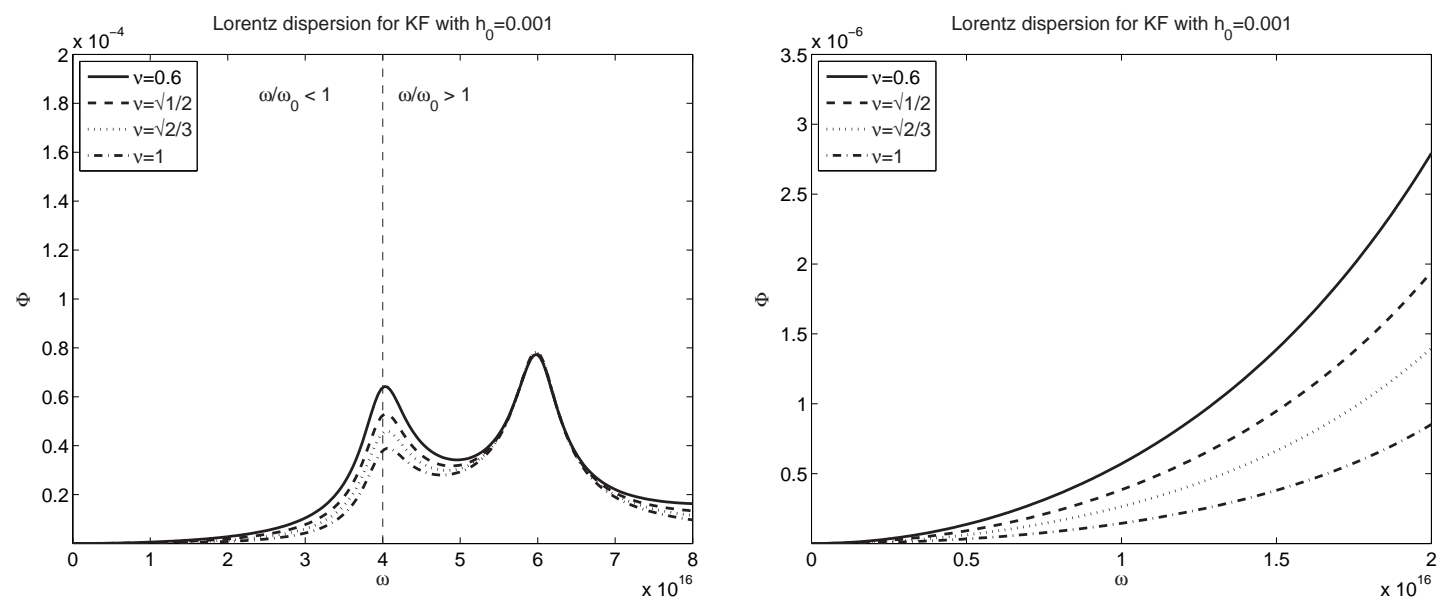

Figure 58: Plot of the phase error $\phi$ versus $\omega$ for the KF finite difference scheme of the Lorentz model with $\nu=\{1, \sqrt{2 / 3}, \sqrt{1 / 2}, .6\}$ using $h_{0}=0.001$. (Right plot is zoom of left plot.)
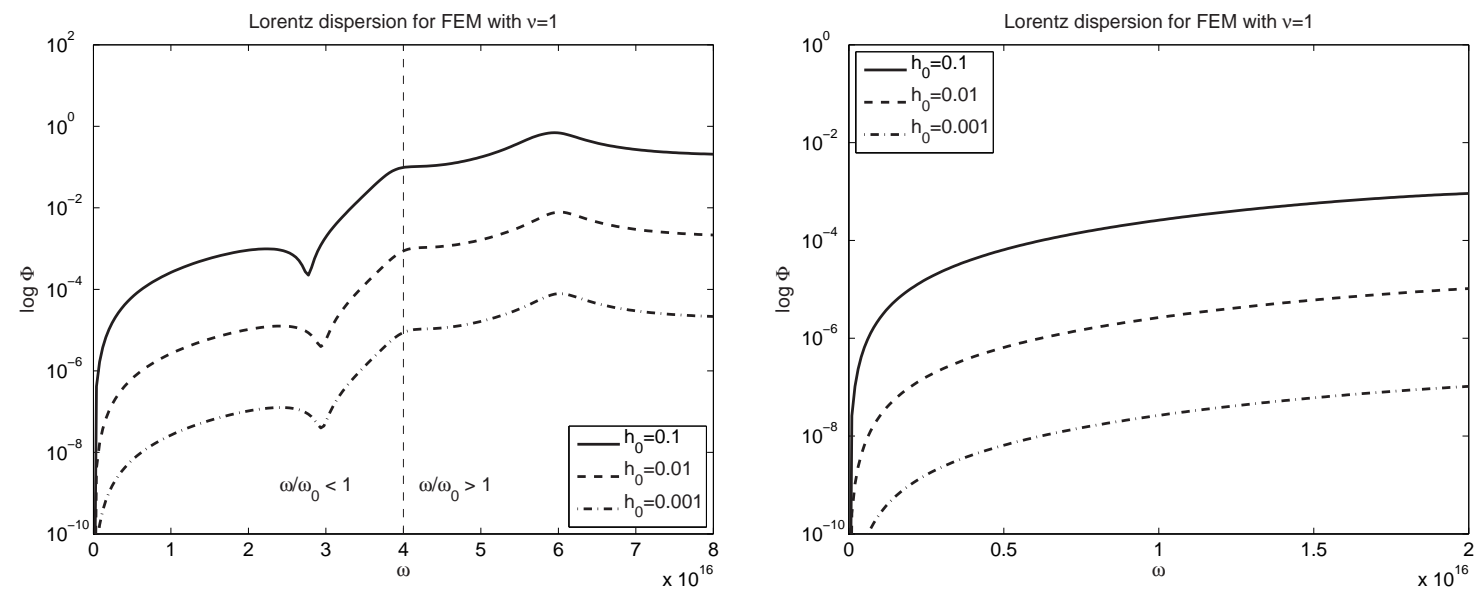

Figure 59: Plot of the $\log$ of the phase error $\phi$ versus $\omega$ for the finite element scheme of the Lorentz model with $\nu=1$ using $h_{0}=\{0.1,0.01,0.001\}$. (Right plot is zoom of left plot.) 

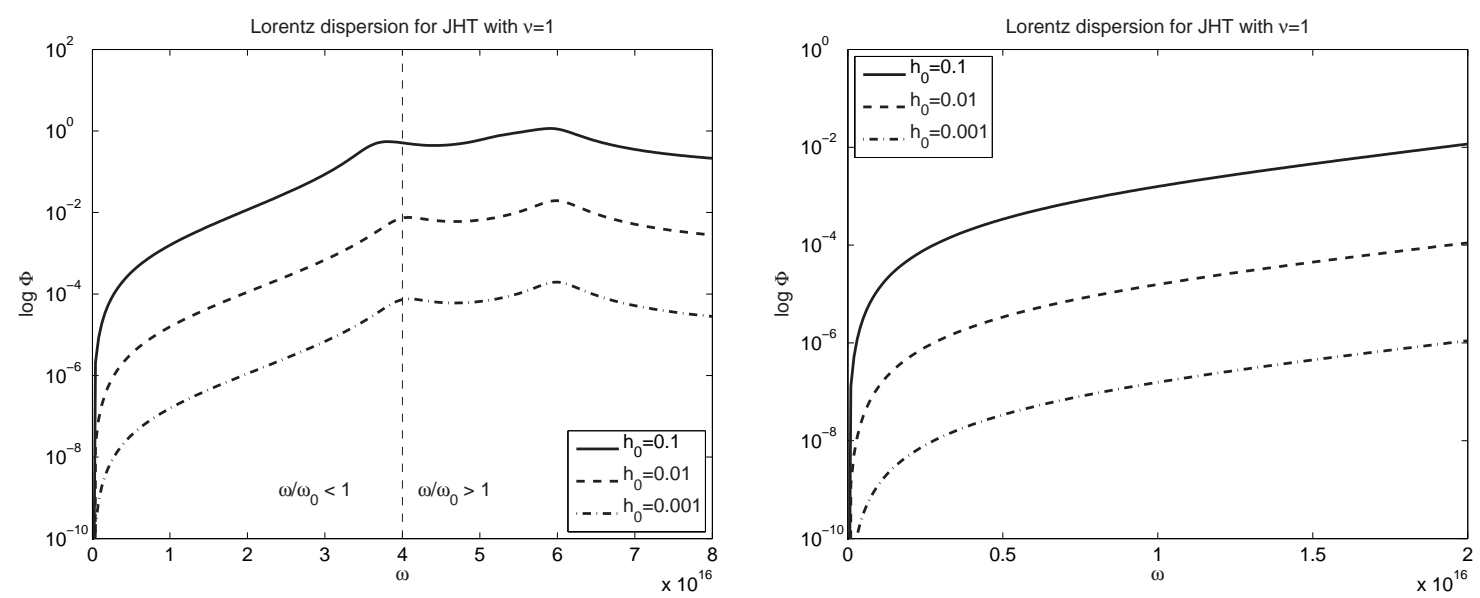

Figure 60: Plot of the log of the phase error $\phi$ versus $\omega$ for the JHT finite difference scheme of the Lorentz model with $\nu=1$ using $h_{0}=\{0.1,0.01,0.001\}$. (Right plot is zoom of left plot.)
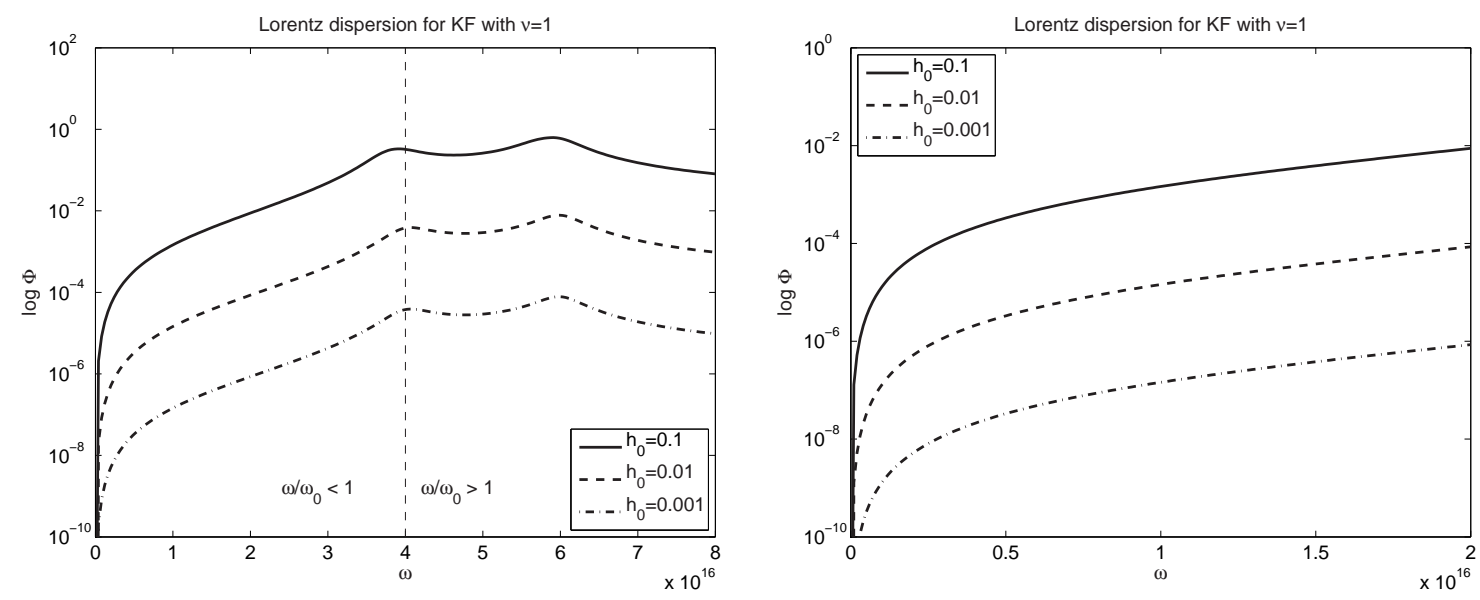

Figure 61: Plot of the $\log$ of the phase error $\phi$ versus $\omega$ for the KF finite difference scheme of the Lorentz model with $\nu=1$ using $h_{0}=\{0.1,0.01,0.001\}$. (Right plot is zoom of left plot.) 


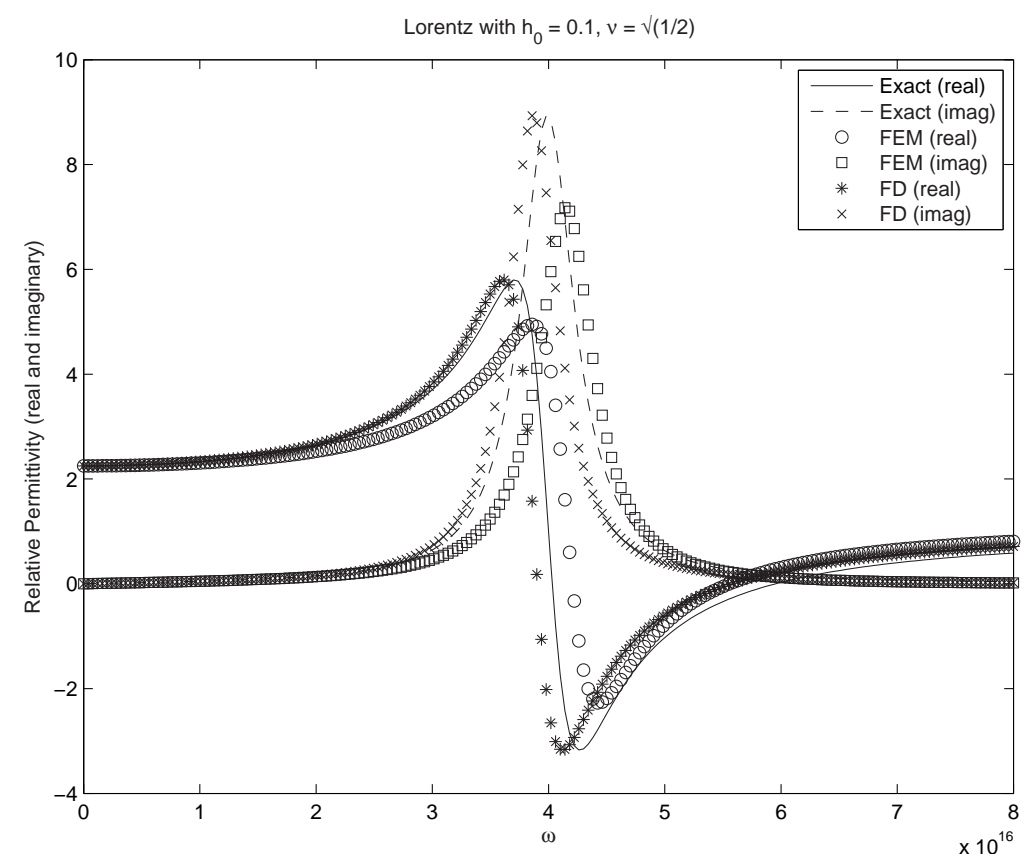

Figure 62: Plots of the real and imaginary parts of the relative complex permittivity for Lorentz media for the continuous equations, the finite element scheme with $\nu=\sqrt{1 / 2}, h_{0}=$ 0.1 and the KF finite difference scheme with $\nu=1, h_{0}=0.1$.

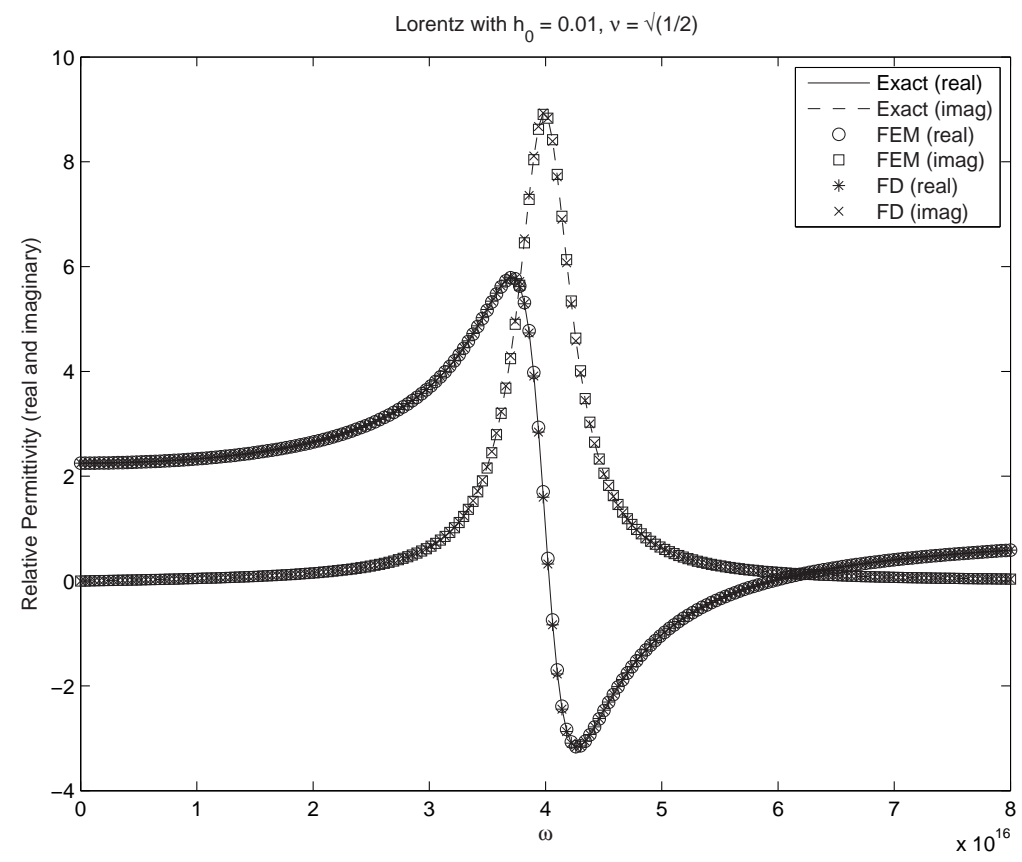

Figure 63: Plots of the real and imaginary parts of the relative complex permittivity for Lorentz media for the continuous equations, the finite element scheme with $\nu=\sqrt{1 / 2}, h_{0}=$ 0.01 and the KF finite difference scheme with $\nu=1, h_{0}=0.01$. 


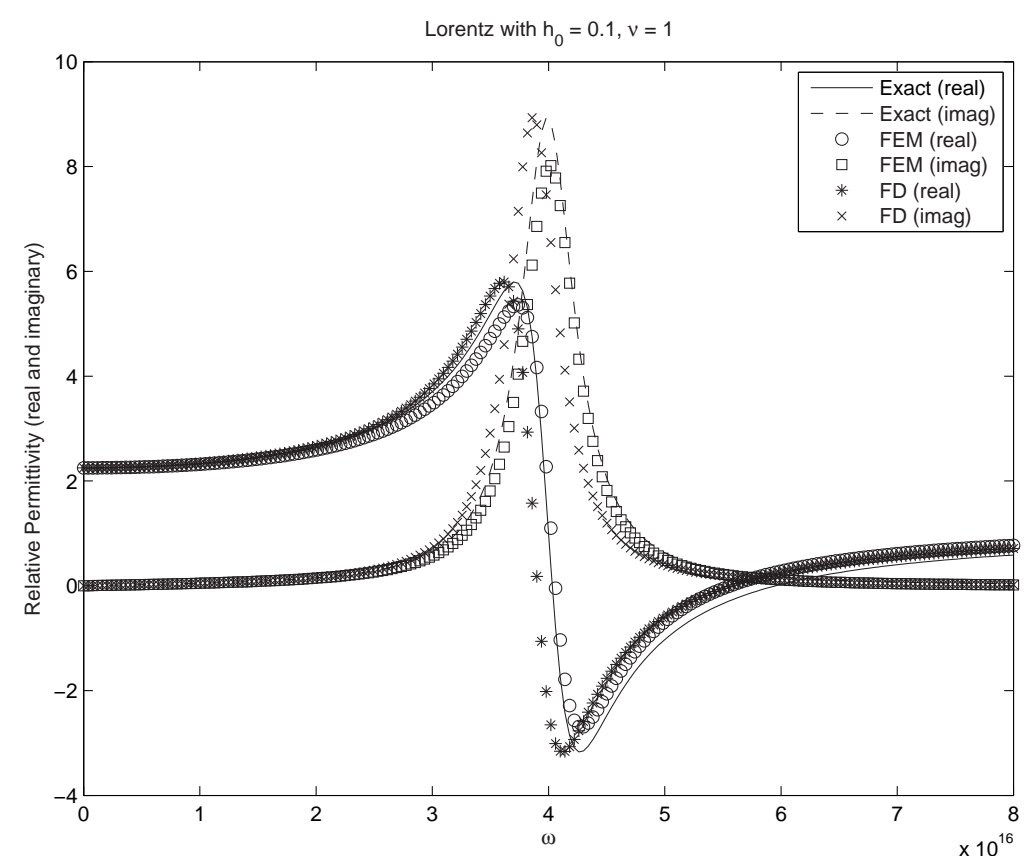

Figure 64: Plots of the real and imaginary parts of the relative complex permittivity for Lorentz media for the continuous equations, the finite element scheme with $\nu=1, h_{0}=0.1$ and the KF finite difference scheme with $\nu=1, h_{0}=0.1$.

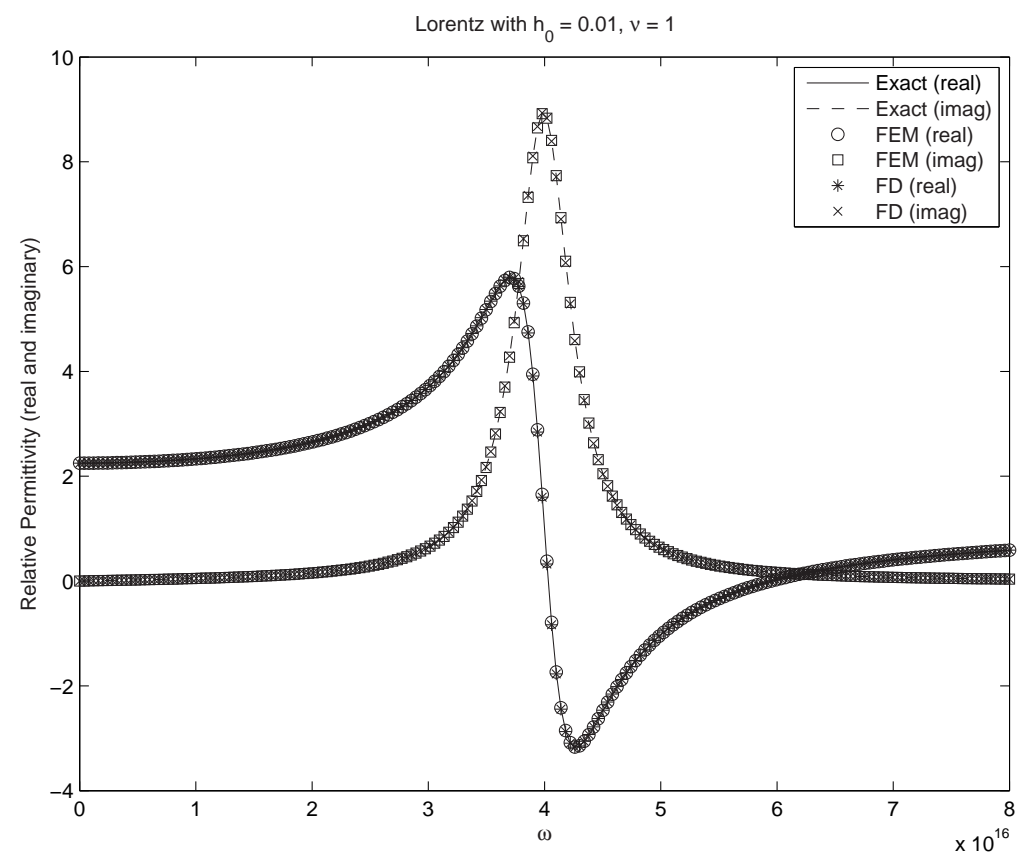

Figure 65: Plots of the real and imaginary parts of the relative complex permittivity for Lorentz media for the continuous equations, the finite element scheme with $\nu=1, h_{0}=0.01$ and the KF finite difference scheme with $\nu=1, h_{0}=0.01$. 


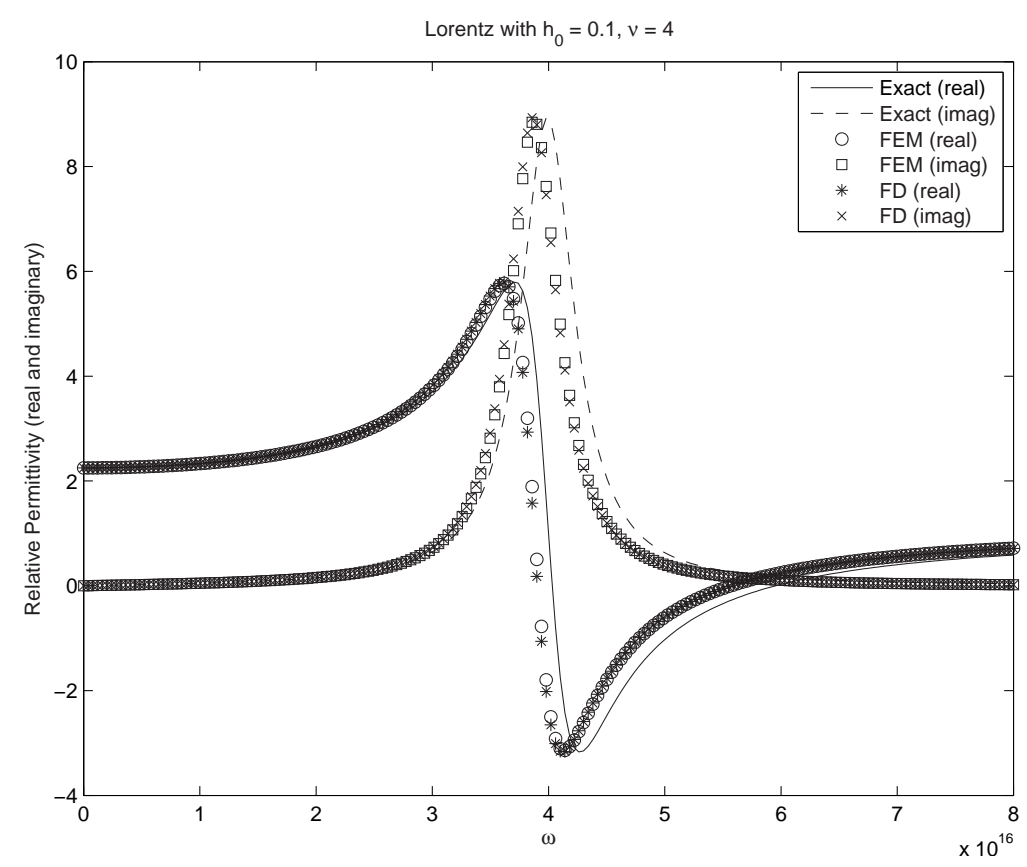

Figure 66: Plots of the real and imaginary parts of the relative complex permittivity for Lorentz media for the continuous equations, the finite element scheme with $\nu=4, h_{0}=0.1$ and the KF finite difference scheme with $\nu=1, h_{0}=0.1$.

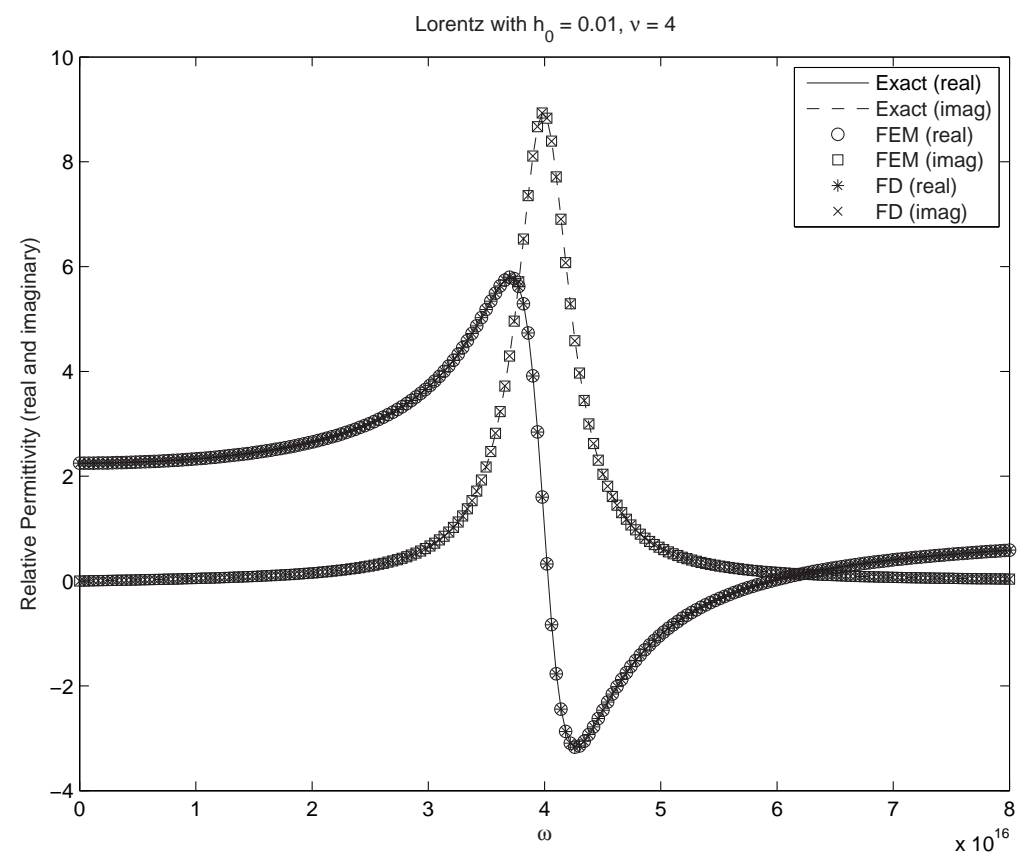

Figure 67: Plots of the real and imaginary parts of the relative complex permittivity for Lorentz media for the continuous equations, the finite element scheme with $\nu=4, h_{0}=0.01$ and the KF finite difference scheme with $\nu=1, h_{0}=0.01$. 

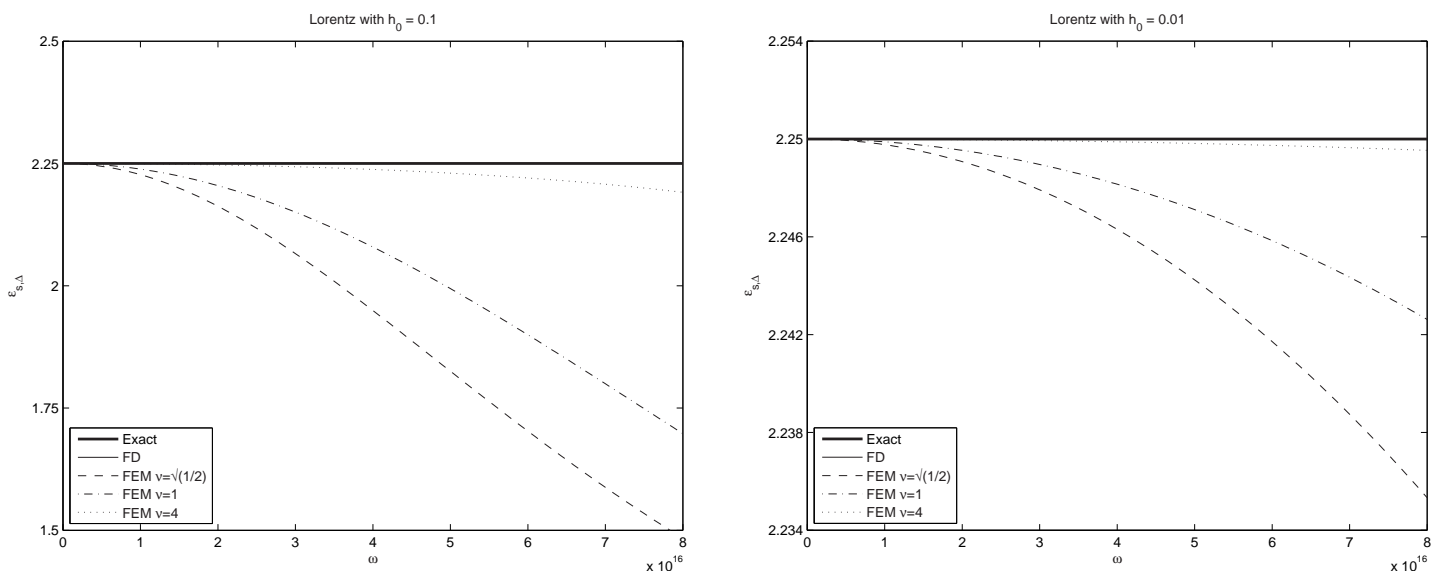

Figure 68: Plots of $\epsilon_{s}$ for Lorentz media for the continuous equations, the finite element scheme and the KF finite difference scheme $(\nu=1)$ with $h_{0}=0.1$ (left) and $h_{0}=0.01$ (right).
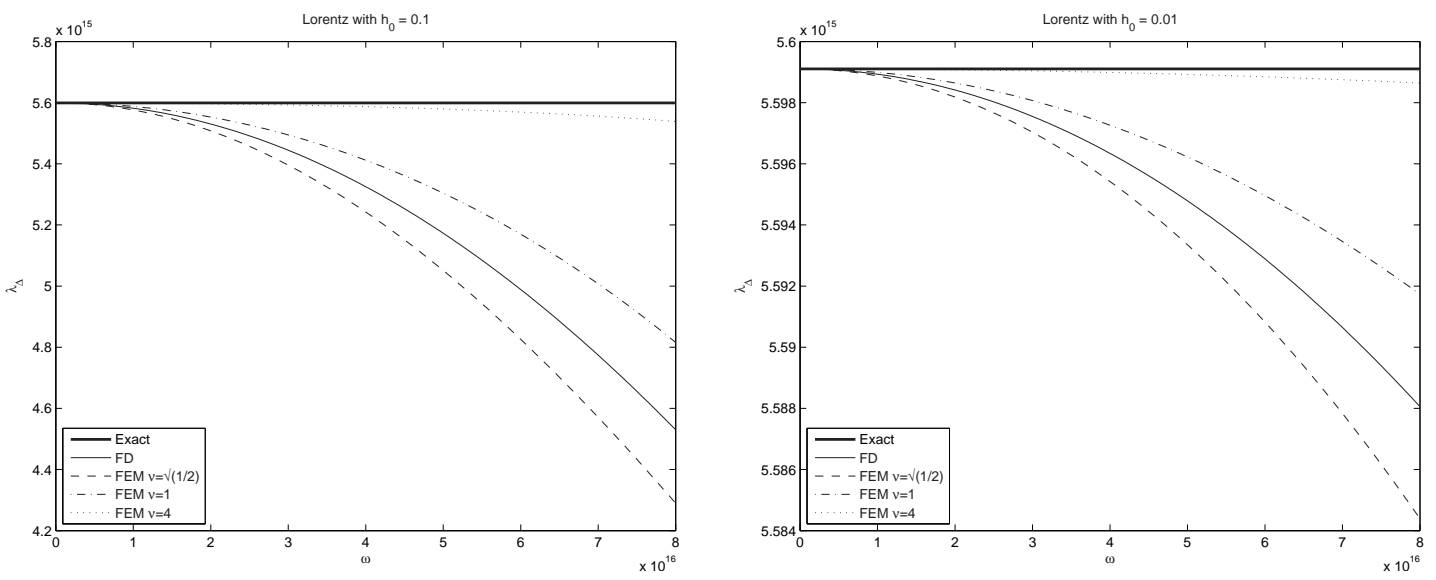

Figure 69: Plots of $\lambda$ for Lorentz media for the continuous equations, the finite element scheme and the KF finite difference scheme $(\nu=1)$ with $h_{0}=0.1$ (left) and $h_{0}=0.01$ (right). 

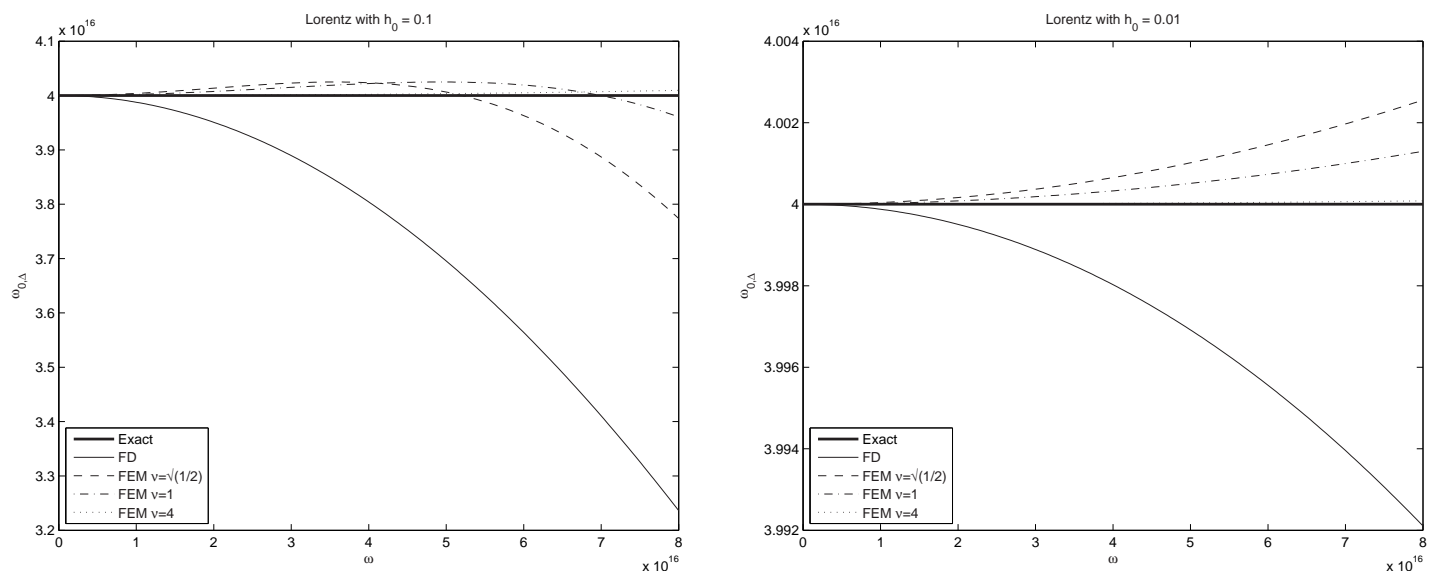

Figure 70: Plots of the resonant frequency $\omega_{0}$ for Lorentz media for the continuous equations, the finite element scheme and the KF finite difference scheme $(\nu=1)$ with $h_{0}=0.1$ (left) and $h_{0}=0.01$ (right).
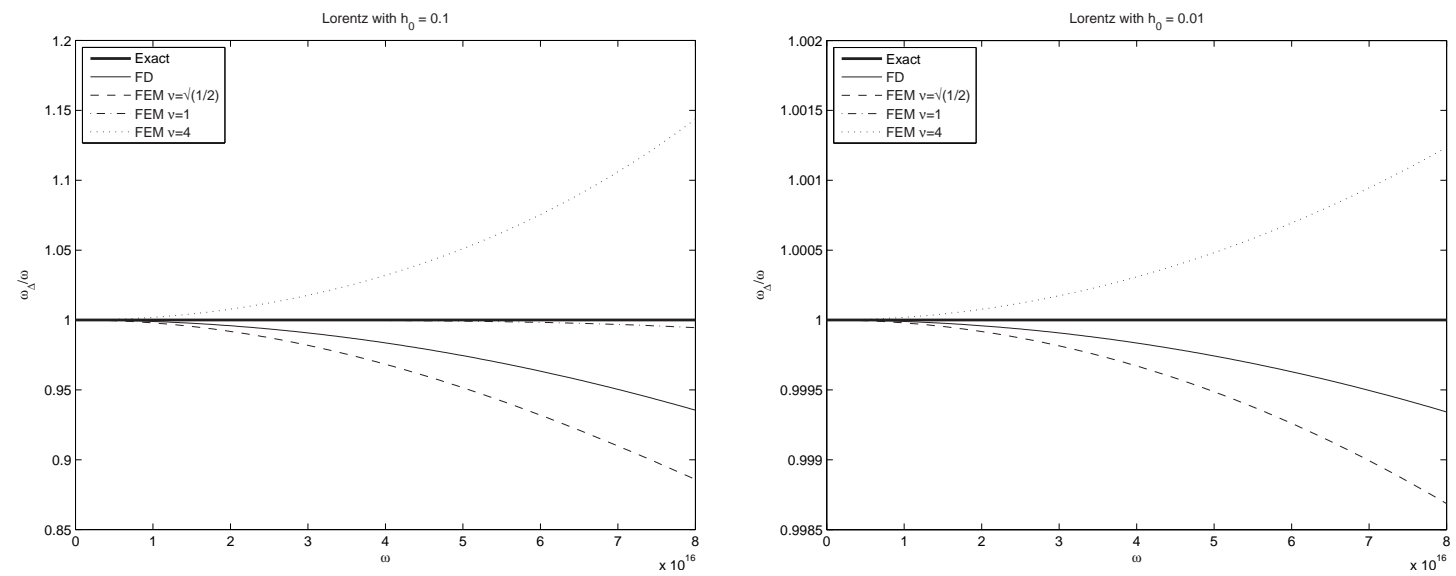

Figure 71: Plots of the frequency $\omega$ for Lorentz media for the continuous equations, the finite element scheme and the KF finite difference scheme $(\nu=1)$ with $h_{0}=0.1$ (left) and $h_{0}=0.01$ (right). 


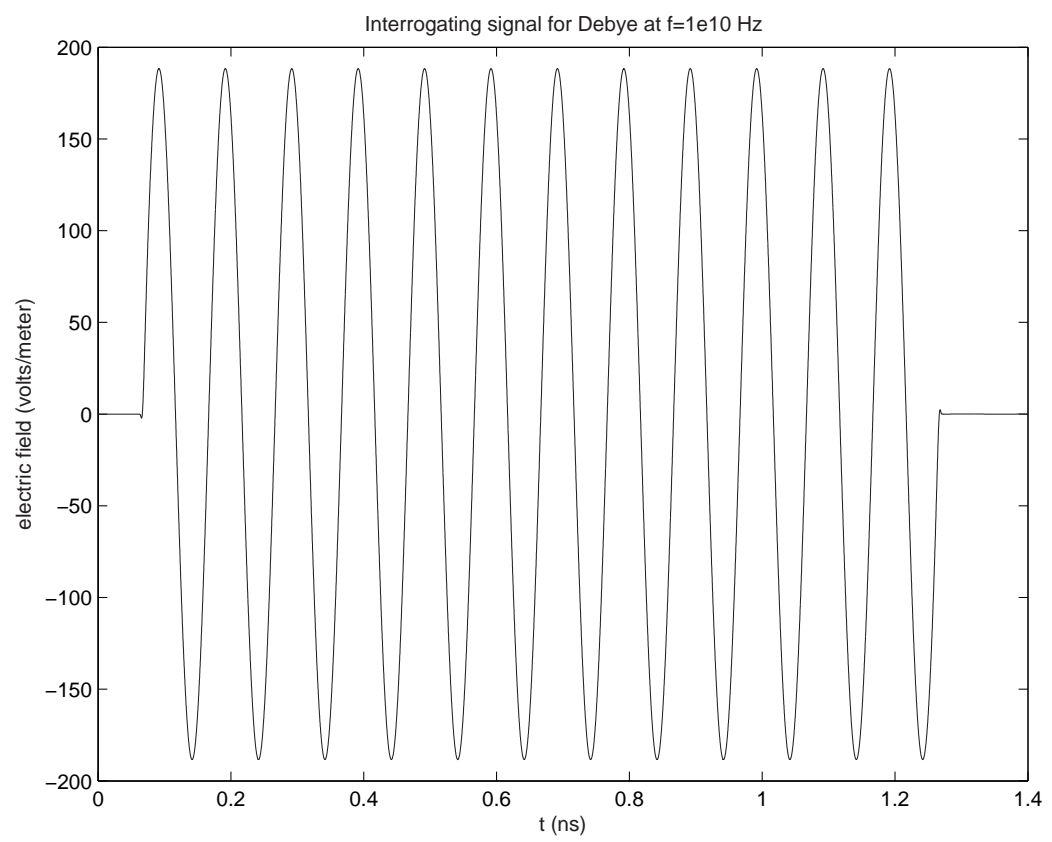

Figure 72: The interrogating signal when the frequency is $10 \mathrm{GHz}$.

\section{Numerical Simulations}

To verify the observations drawn from the stability and dispersion analyses in the previous sections, we examine simulations of several sample problems, which use either the Debye polarization model or the Lorentz model. In each case we will successively reduce the value of $h_{\tau}$, and hence the time step, holding all other parameters fixed, until convergence is achieved.

\subsection{Debye Simulations}

In our first numerical calculation we simulate the propagation of 12 cycles of a truncated sine wave with carrier frequency at $10 \mathrm{GHz}\left(1 \times 10^{10} \mathrm{~Hz}\right)$, plotted in Figure 72 , which is normally incident on a Debye medium from a vacuum. The medium is defined by the parameters given in Section 4.2, namely $\epsilon_{s}=78.2, \epsilon_{\infty}=1$, and $\tau=8.1 \times 10^{-12}$ seconds. We performed simulations with the finite element scheme using $\nu=4$, as the dispersion analysis demonstrated that for this value of $\nu$ we obtained the least phase error in the regime that is of interest in this simulation. A time trace of the electric field at a depth of $15 \mathrm{~mm}$ was recorded. Figure 73 shows the solutions for three different values of $h_{\tau}$. The traces for $h_{\tau}=0.16$ and $h_{\tau}=0.08$ appear to be identical at this scale. However, Figure 74 , which displays a closer view of the central portion of the signal, demonstrates that true convergence has not been reached. Figure 75 displays plots of one of the peaks from the central portion of the signal with smaller $h_{\tau}$ values. From this plot we can see that at most $h_{\tau}=0.01$ (i.e., at least 100 points per $\tau$ ) is necessary for convergence on this small scale. 


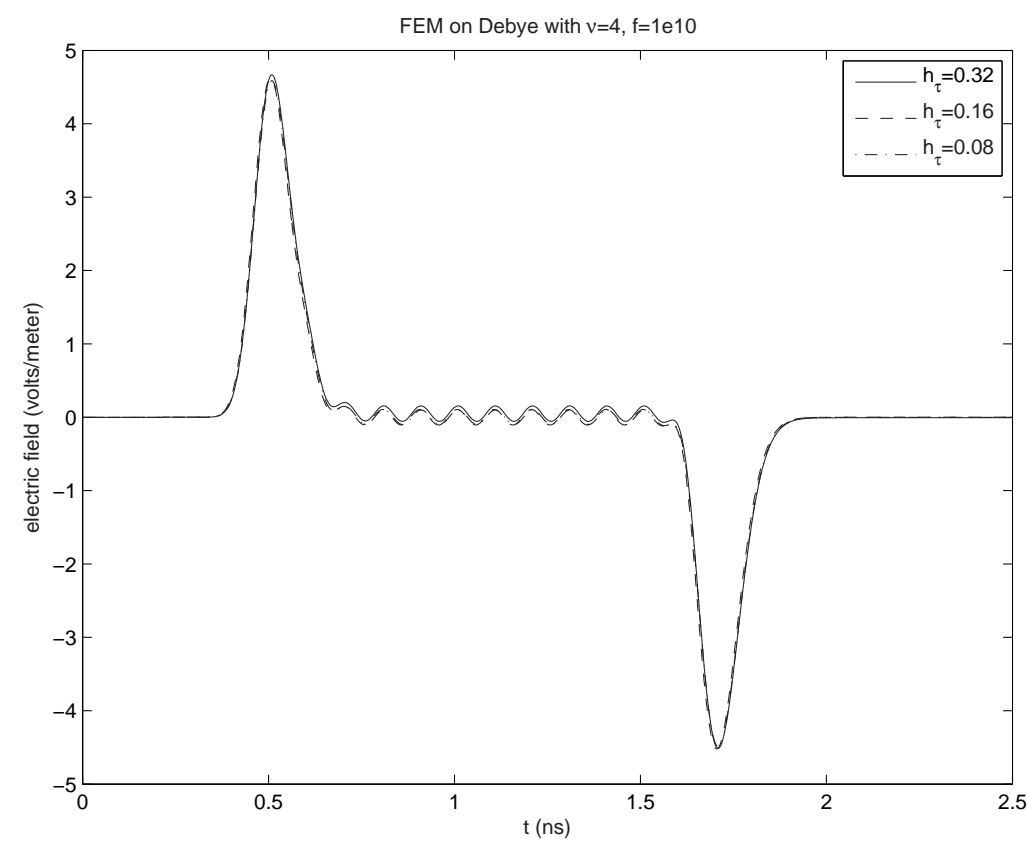

Figure 73: Time trace of the electric field at a depth of $15 \mathrm{~mm}$ into a Debye medium (frequency is $10 \mathrm{GHz}$ ) computed using the finite element scheme, for three different values of $h_{\tau}$.

We note that while this frequency is "relatively" small (i.e., $\omega \tau=0.5089<1$ ), and therefore the corresponding points per wavelength is large in this example $(p p w=873)$, however this high resolution of the wavelength is not the reason that the value of $h_{\tau}=0.01$ is required. In fact, for a "relatively" high frequency of $100 \mathrm{GHz}$ (i.e., $\omega \tau=5.089>1$, $p p w=87.3)$, we have essentially the same restriction. Figure 72 plots the interrogating signal when the frequency is $100 \mathrm{GHz}$. Figure 77 shows the solutions for three different values of $h_{\tau}$. Again, the curves corresponding to the smaller two values appear identical. However, Figure 78 shows that at most $h_{\tau}=0.01$ is necessary for convergence on this small scale. In this example, the points per $\tau$ are on the same order of magnitude as the points per wavelength. Any further increase in frequency would result in the period of the interrogating signal, $T$, being much less than the relaxation time, and therefore chosing $h_{\tau}=0.01$ would not guarantee that the smallest time scale in the problem is resolved.

These examples verify the guideline of at least 100 points per relaxation time, when $\tau$ is the smallest time scale to be resolved. This agrees with FDTD results from [28], in that to ensure minimal dispersion and dissapation error, $h_{\tau}$ should be less than 0.01 , preferably $O\left(10^{-3}\right)$. As the same number of time steps are required for both the finite element method and the finite difference method, the fact that the finite element method used here requires a linear solve at each time step is an issue. However, both of the above simulations for interrogating a Debye material using $h_{\tau}=0.01$ took less than 300 seconds on a $3.8 \mathrm{GHz}$ speed processor. The case when $h_{\tau}=0.16$ takes only .3 seconds. In order to compare the stability and phase errors between the finite element method and the finite difference 


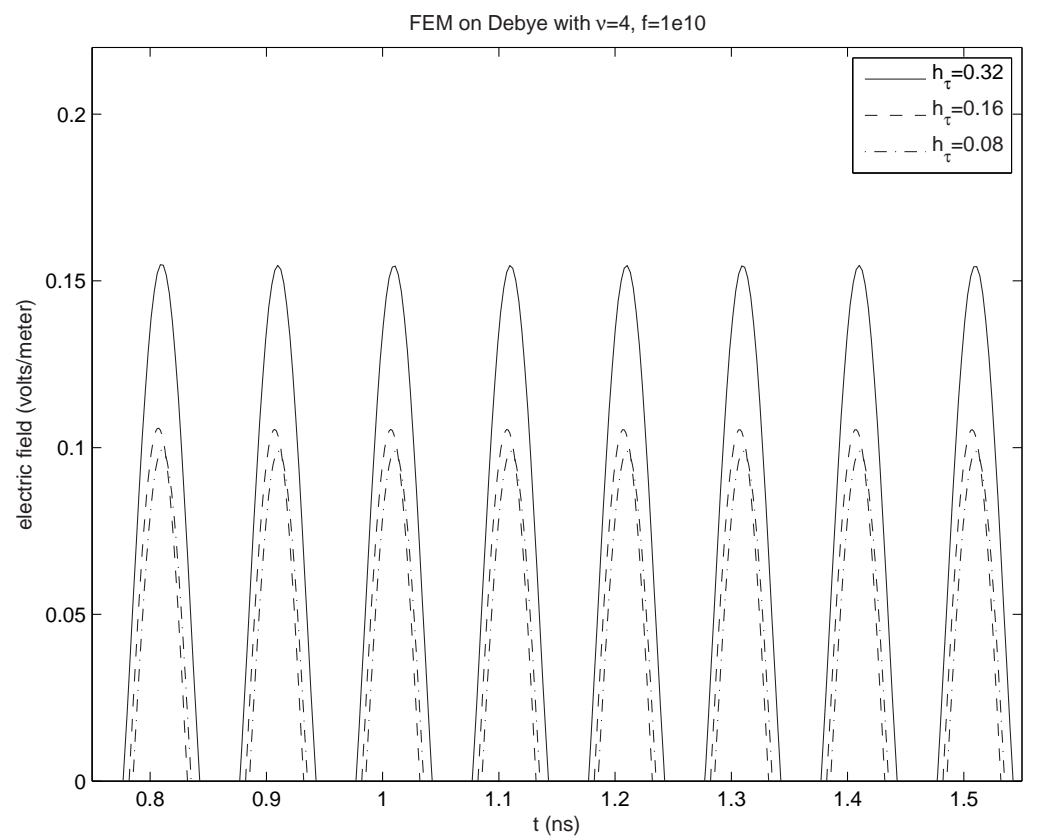

Figure 74: Closer view of the center of the time trace of the electric field at a depth of 15 $\mathrm{mm}$ into a Debye medium (frequency is $10 \mathrm{GHz}$ ), for three smaller values of $h_{\tau}$.

method, we first determine that $h_{\tau}=0.04$ requires 0.3 seconds and $h_{\tau}=0.0015$ requires 280 seconds. Figure 79 compares the stability and phase errors of the finite element method and the finite difference method for the parameter set that resulted in a 0.3 second runtime. Figure 80 displays the same comparisons for the 280 second runtime case. The finite element method has the lesser dispersion error only for the low frequency and low resolution case. However, the stability results and phase errors for the other cases are all on the same order of magnitude as the finite difference method. 


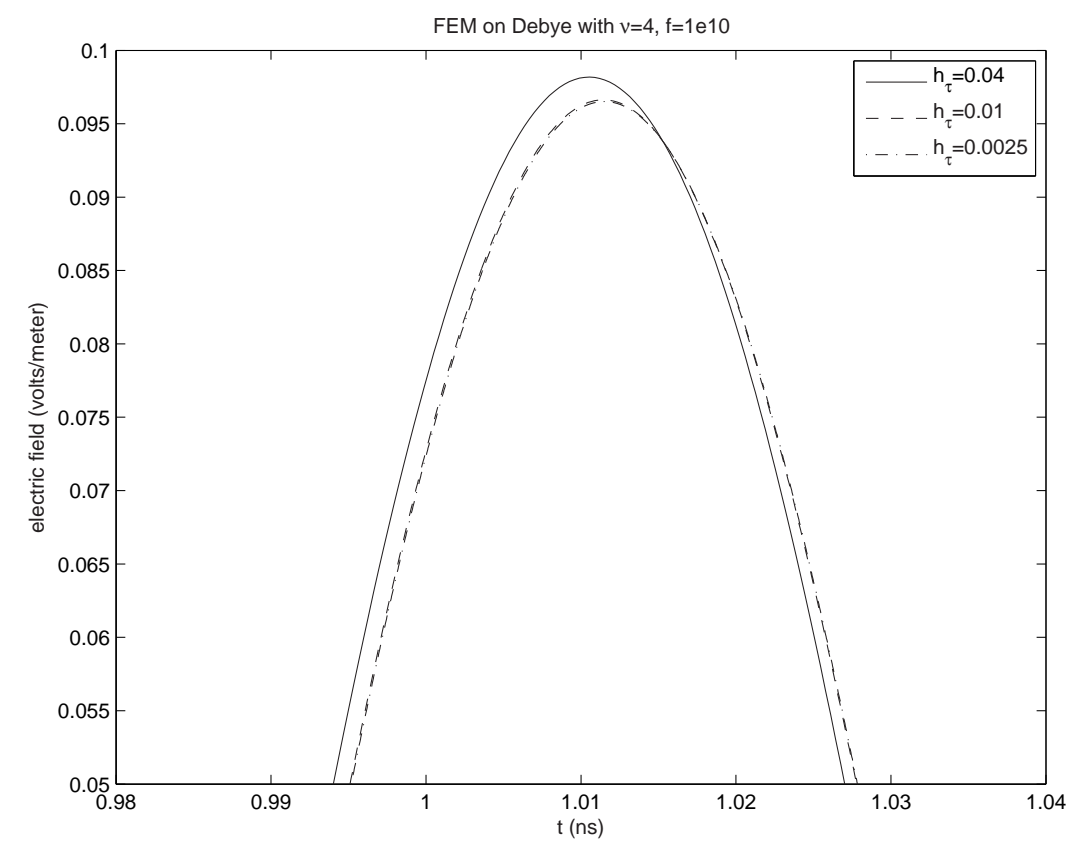

Figure 75: Magnified view of the time trace of the electric field at a depth of $15 \mathrm{~mm}$ into a Debye medium (frequency is $10 \mathrm{GHz}$ ). Convergence on this scale is achieved at $h_{\tau}=0.01$.

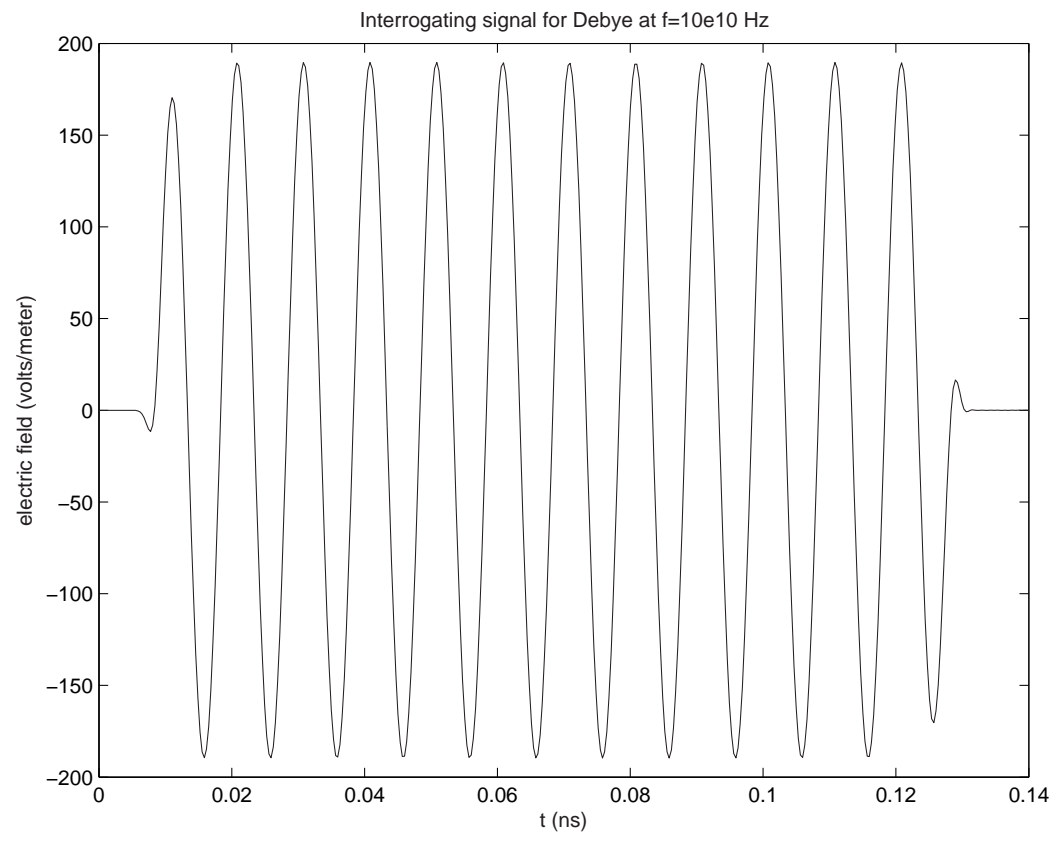

Figure 76: The interrogating signal when the frequency is $100 \mathrm{GHz}$. 


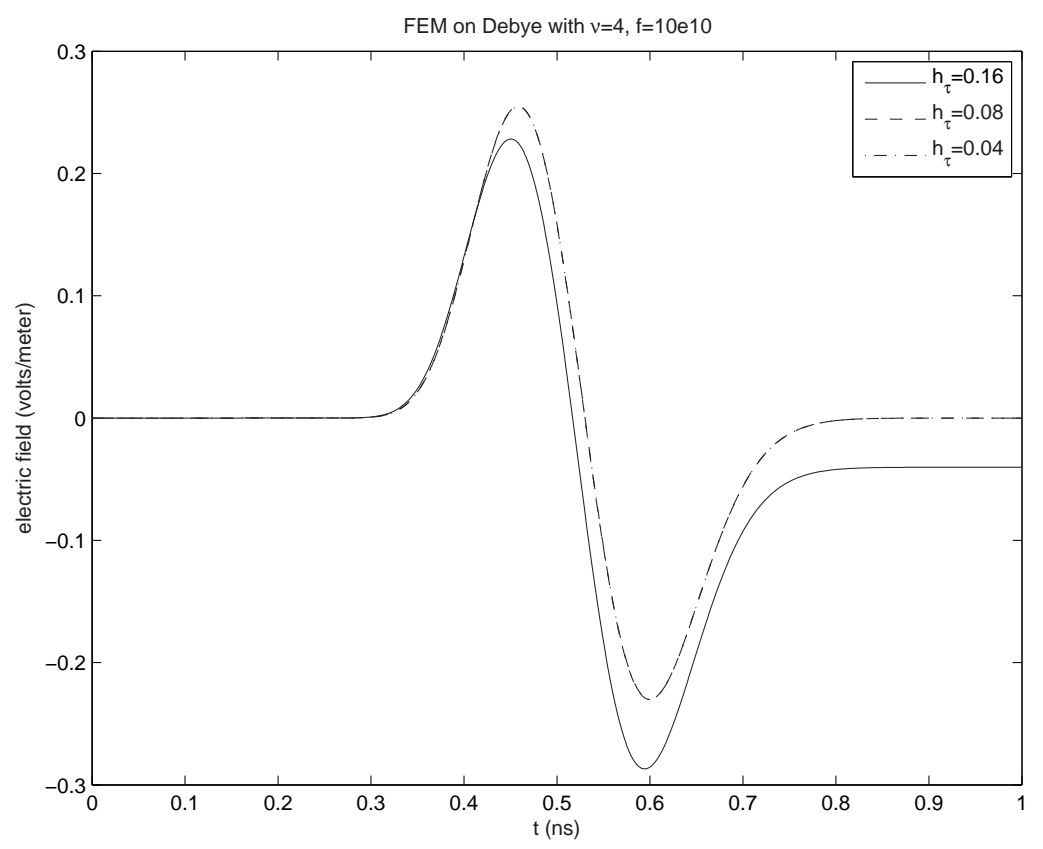

Figure 77: Time trace of the electric field at a depth of $15 \mathrm{~mm}$ into a Debye medium (frequency is $100 \mathrm{GHz}$ ) computed using the finite element scheme, for three different values of $h_{\tau}$.

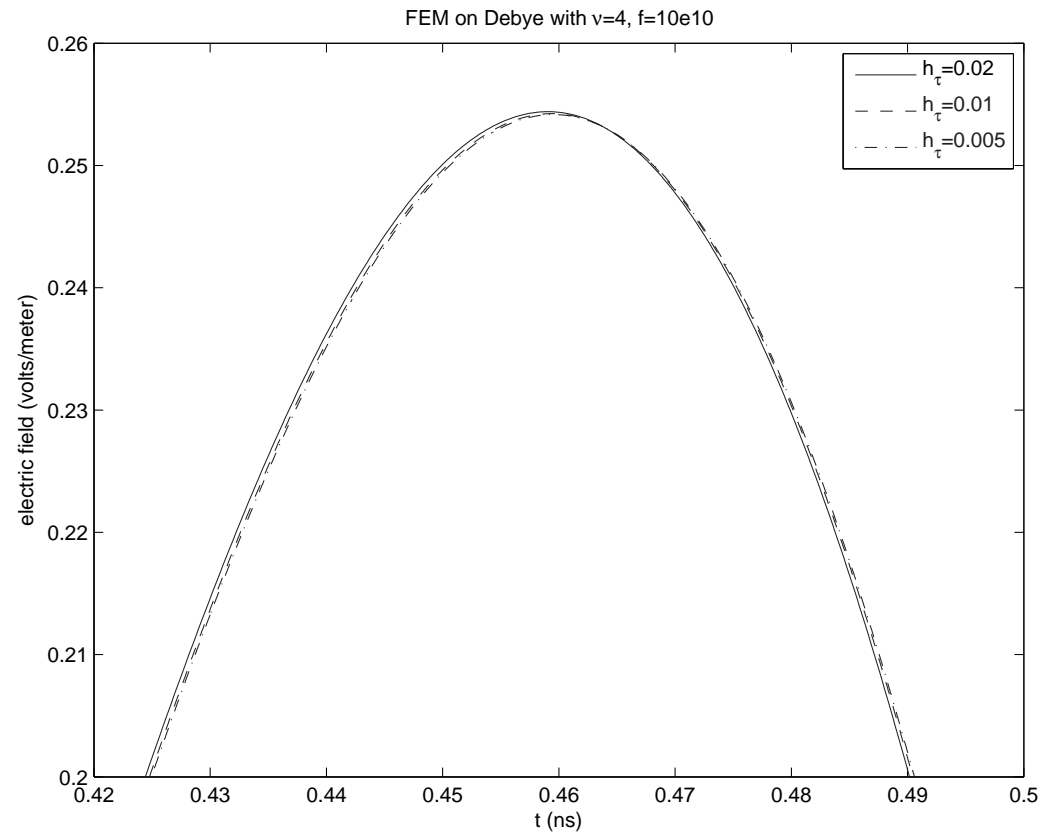

Figure 78: Magnified view of the time trace of the electric field at a depth of $15 \mathrm{~mm}$ into a Debye medium (frequency is $100 \mathrm{GHz}$ ). Convergence on this scale is achieved at $h_{\tau}=0.01$. 

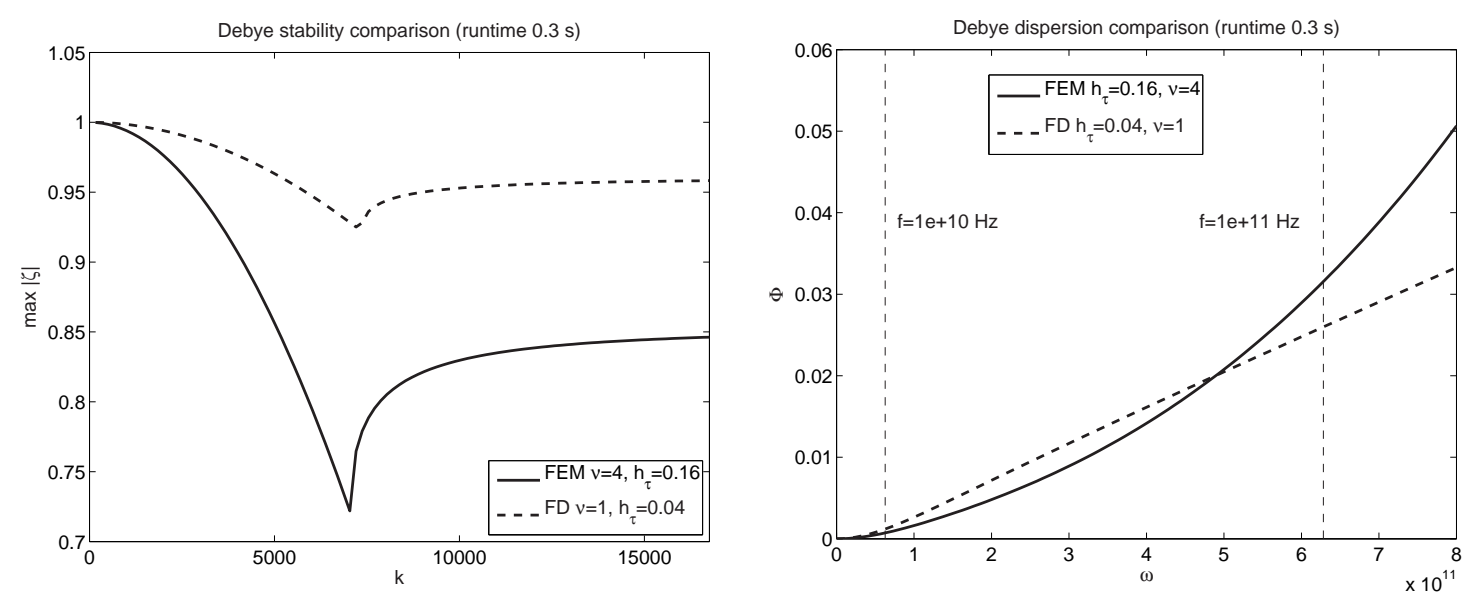

Figure 79: The stability and phase errors of the finite element method and the finite difference method applied to the Debye model for the parameter set that resulted in a 0.3 second runtime.
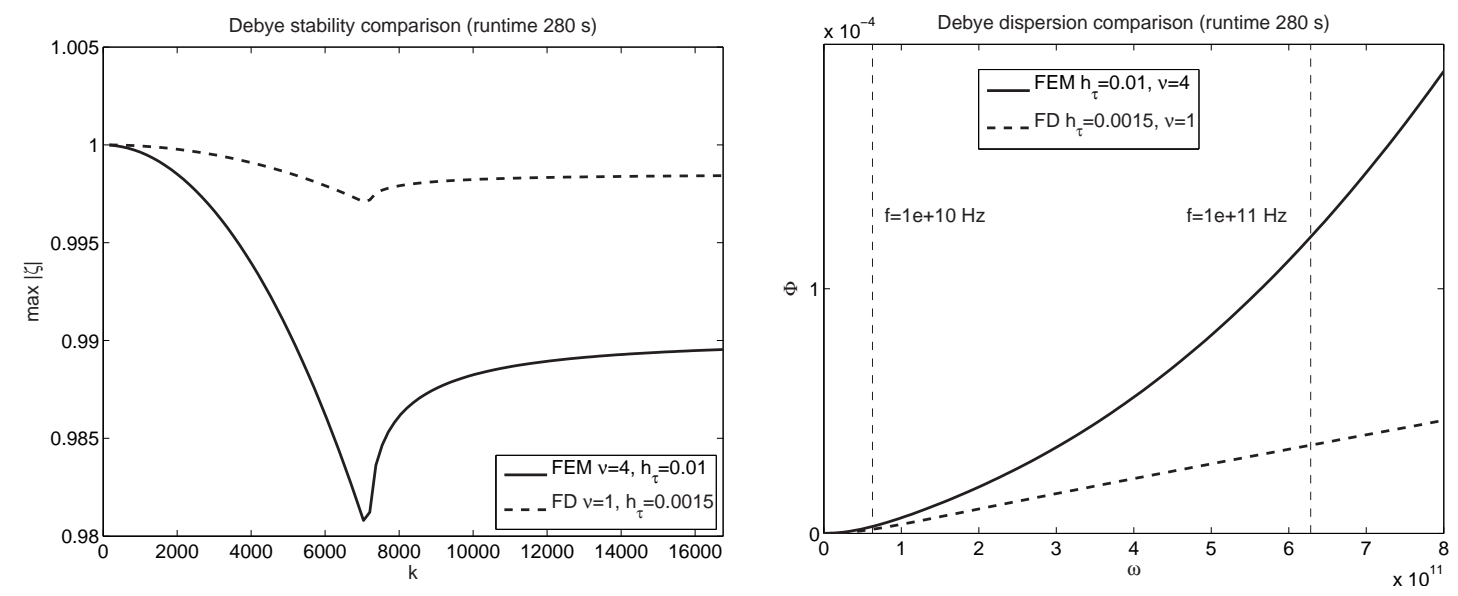

Figure 80: The stability and phase errors of the finite element method and the finite difference method applied to the Debye model for the parameter set that resulted in a 280 second runtime. 


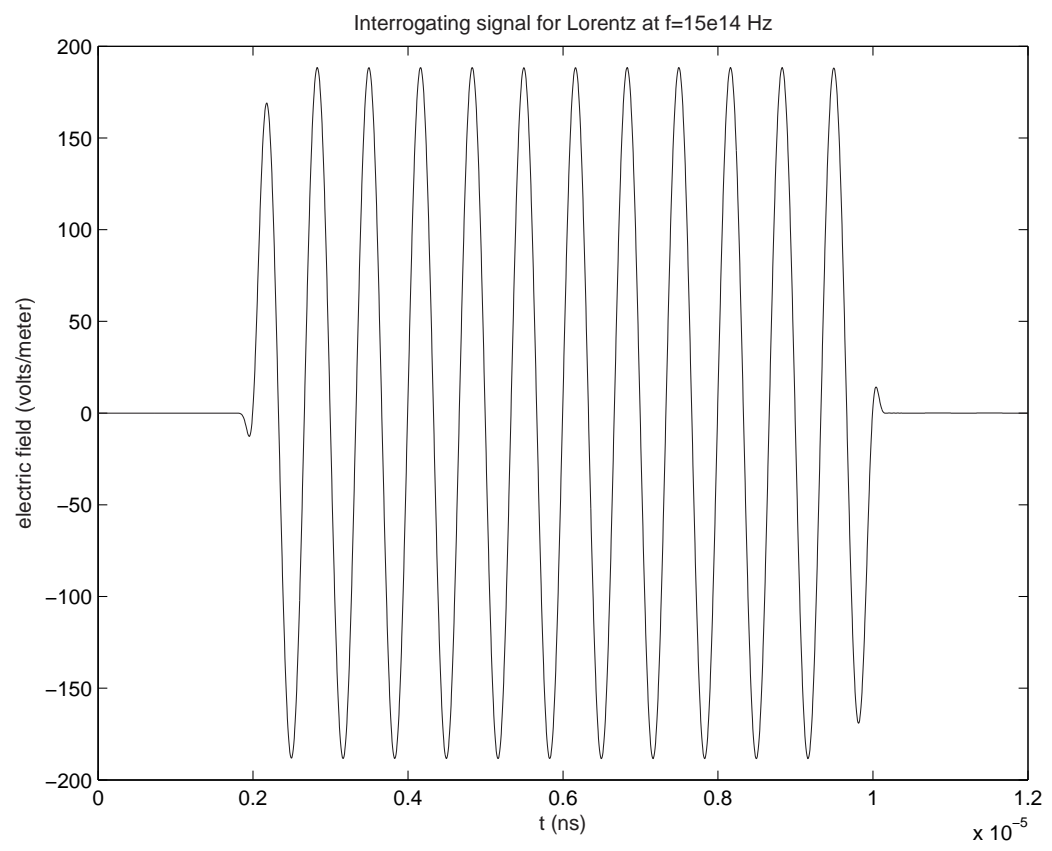

Figure 81: The interrogating signal when the frequency is $1.5 \mathrm{PHz}$.

\subsection{Lorentz Simulations}

For interrogation of a Lorentz medium, we simulate the propagation of 12 cycles of a truncated sine wave with carrier frequency at $1.5 \mathrm{PHz}\left(1.5 \times 10^{15} \mathrm{~Hz}\right)$, plotted in Figure 81 , which is normally incident on the medium from a vacuum. The medium is defined by the parameters given in Section 4.3, namely $\epsilon_{s}=2.25, \epsilon_{\infty}=1, \tau=1.786 \times 10^{-16}$, and $\omega_{0}=4 \times 10^{16}$. We performed simulations with the finite element scheme using $\nu=1$. A time trace of the electric field at a depth of $0.01 \mathrm{~mm}$ was recorded. Figure 82 shows the solutions for three different values of $h_{\tau}$. The traces for $h_{0}=0.16$ and $h_{0}=0.08$ appear to be identical at this scale. However, Figure 83, which displays a closer view of the central portion of the signal, demonstrates that true convergence has not been reached. Figure 84 displays plots of one of the peaks from the central portion of the signal with smaller $h_{0}$ values. From this plot we can see that at most $h_{0}=0.02$ (i.e., at least 50 points per $\omega_{0}$ ) is necessary for convergence on this small scale.

The required value of $h_{0}<0.02$ is in line with the suggested range of $O\left(10^{-2}\right)$. This also agrees with FDTD results from [28].

As we have simulated propagation deeper (w.r.t wavelengths) into the material in this case as compared with the Debye simulations above (6600 spatial steps, i.e. 50 wavelengths into the material versus 437 spatial steps or 0.5 wavelength for the Debye medium), our computational time is appropriately longer. For $h_{0}=0.02$, the runtime was greater than 10 minutes on a $3.8 \mathrm{GHz}$ speed processor.

Again, the finite element method used here requires a linear solve at each time step as opposed to both finite difference methods. Thus, in order to compare the stability plots 


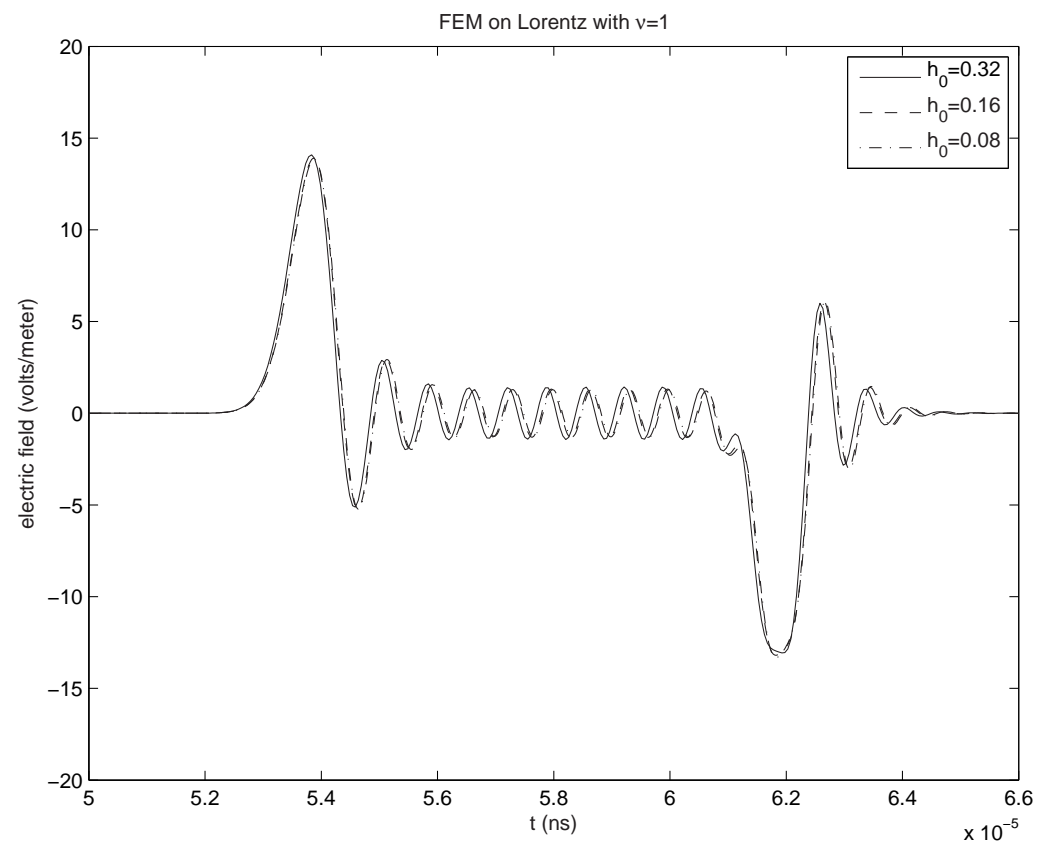

Figure 82: Time trace of the electric field at a depth of $0.01 \mathrm{~mm}$ into a Lorentz medium computed using the finite element scheme, for three different values of $h_{0}$.

and phase errors of the finite element method to the finite difference method (here, the $\mathrm{KF}$ scheme), we first determine the value of $h_{0}$ in the KF scheme which gives runtimes comparable to those of the cases with $h_{0}=0.16$ and $h_{0}=0.02$ when used in the finite element method. The runtimes are 10 seconds and 780 seconds, respectively, and the corresponding $h_{0}$ values are 0.03 and 0.0038 on a $3.8 \mathrm{GHz}$ speed processor. Figure 85 compares the stability and phase errors of the finite element method and the finite difference method for the parameter set that resulted in a 10 second runtime. Figure 86 displays the same comparisons for the 780 second runtime case. Similar to the Debye case, the stability results and phase errors are all on the same order of magnitude as in the finite difference method. 


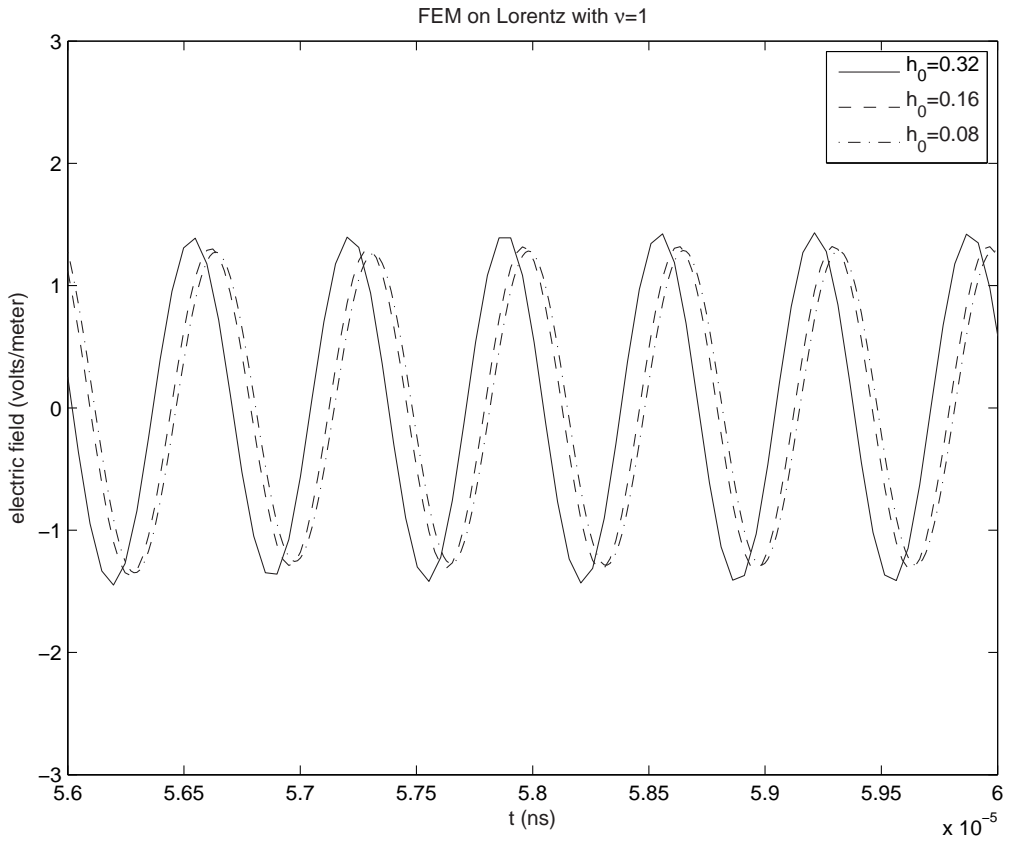

Figure 83: Closer view of the center of the time trace of the electric field at a depth of 0.01 mm into a Lorentz medium, for three smaller values of $h_{0}$.

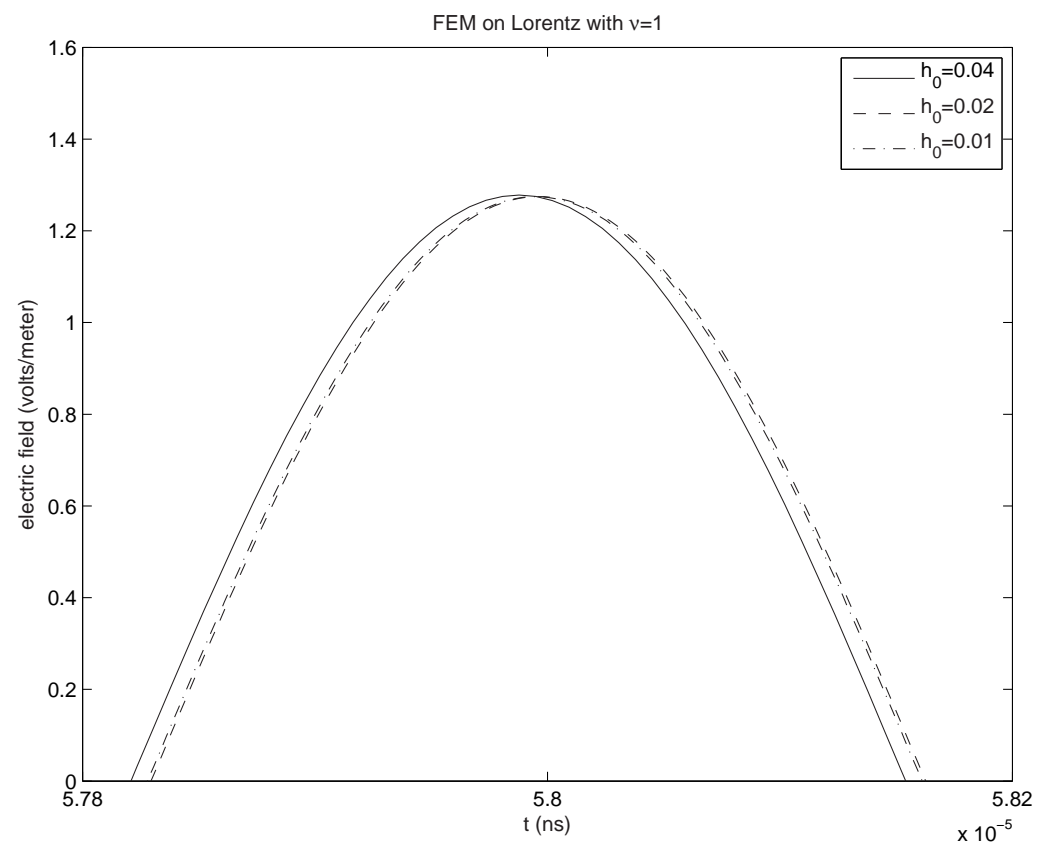

Figure 84: Magnified view of the time trace of the electric field at a depth of $0.01 \mathrm{~mm}$ into a Lorentz medium. Convergence on this scale is achieved at $h_{\tau}=0.02$. 

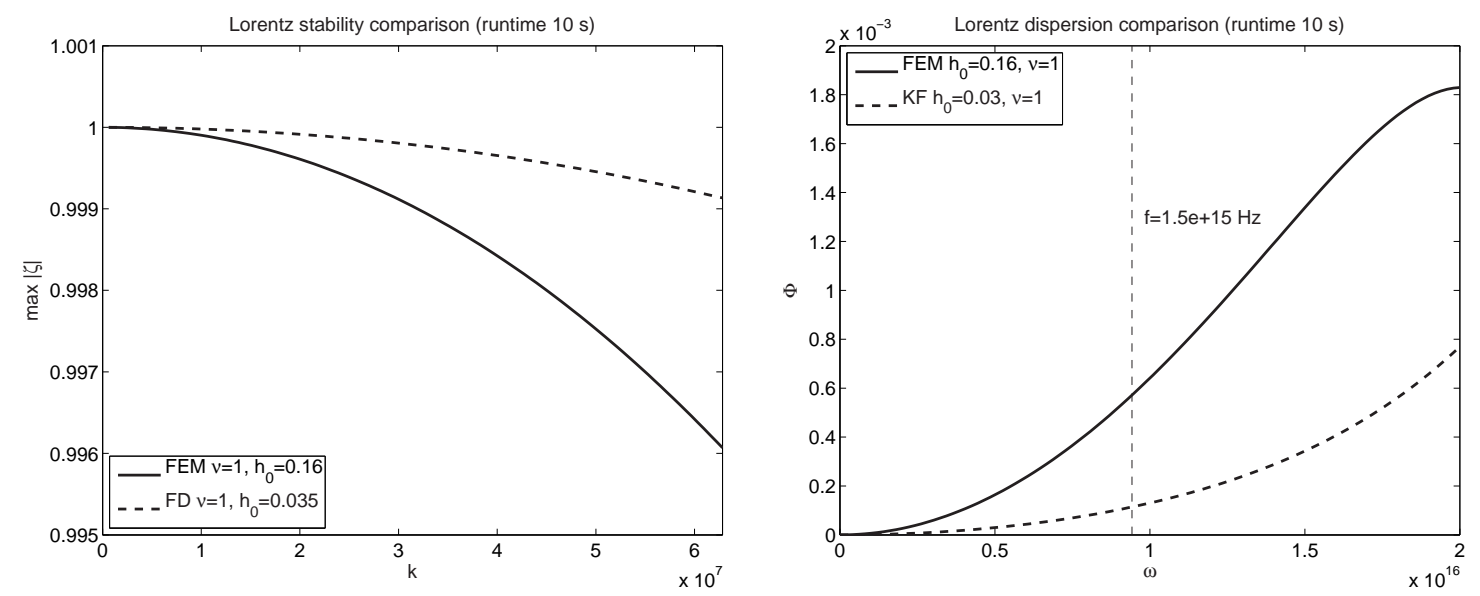

Figure 85: The stability and phase errors of the finite element method and the finite difference method applied to the Lorentz model for the parameter set that resulted in a 10 second runtime.
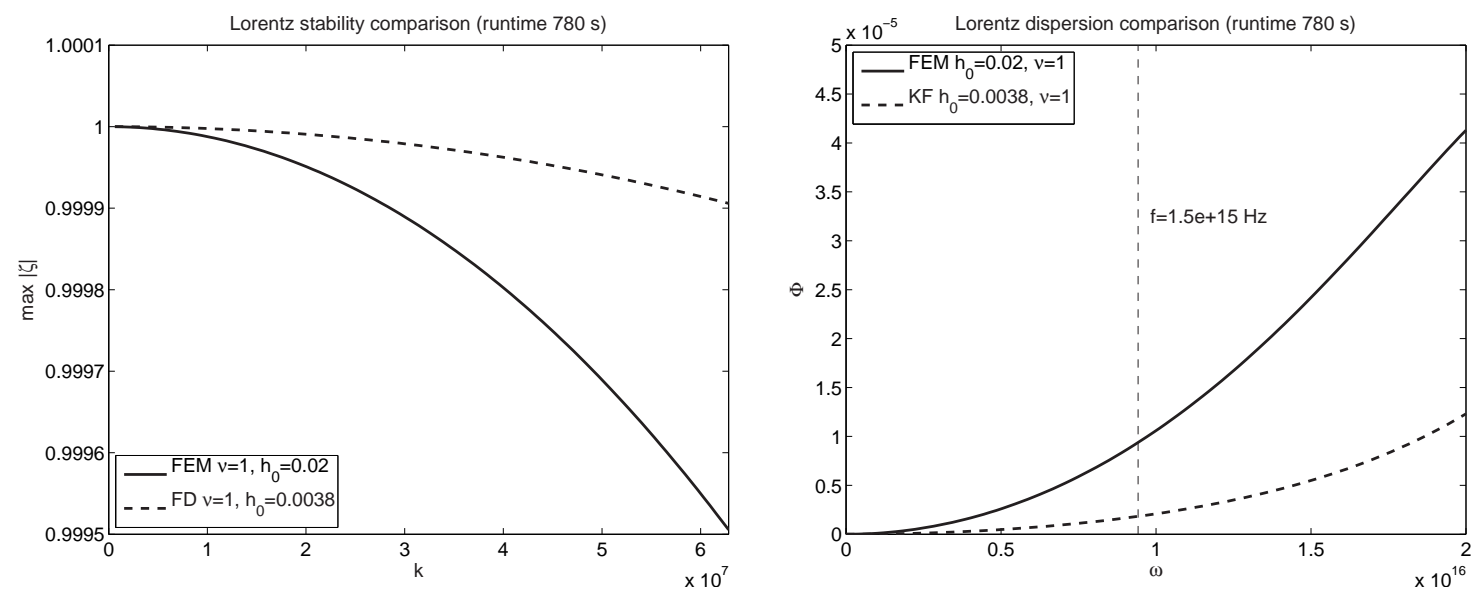

Figure 86: The stability and phase errors of the finite element method and the finite difference method applied to the Lorentz model for the parameter set that resulted in a 780 second runtime. 


\section{Conclusions}

From the stability and dispersion analysis as well as the simulations shown in the paper, we can conclude that the artificial dissipation in the finite element schemes presented here for Debye and Lorentz media are strongly dependent on the quantity $h_{\tau}$ when $\tau$ is the smallest time scale. For Lorentz media, in addition, the quantity $h_{0}$ may be the dominant quantity if $T_{0}=2 \pi / \omega_{0}$ is smaller than $\tau$. We see that $h_{\tau}$ or $h_{0}$ have to be sufficiently small so that not much energy is lost after the large amount of timesteps usually needed to propagate short pulses any appreciable distance into a medium. For Debye media $h_{\tau}$ is recommended to be at least 100 points per $\tau$, preferably $h_{\tau}=O\left(10^{-3}\right)$. For Lorentz media we recommend either $h_{0}$ or $h_{\tau}$ to be $O\left(10^{-2}\right)$ in order to minimize dissipation. These results agree with the guidelines posed in [28] for FDTD schemes.

From the dispersion analysis for the finite element schemes we see that the value of $\nu$ to be chosen should be the one that provides the best agreement of the discrete relative complex permittivity with the exact complex permittivity, i.e. the value that has the least phase error for the regime of interest. The unconditional stability of the finite element scheme allows the user to have some freedom in the choice of $\nu$. For the Debye model, the value of $\nu$ that best represents $\lambda$ should sufficiently model the complex permittivity. For the Lorentz model, the value of $\nu$ that correctly represents $\omega$ should be chosen.

\section{Acknowledgments}

This research was supported in part by the U. S. Air Force Office of Scientific Research under grant AFOSR FA9550-04-1-0220 and in part by the National Institute of Aerospace (NIA) and NASA under grant NIA/NCSU-03-01-2536-NC.

\section{References}

[1] R. A. Albanese, H. T. Banks, and J. K. Raye. Nondestructive evaluation of materials usig pulsed microwave interrogating signals and acoustic wave induced reflections. Inverse Problems, 18:1935-1958, 2002.

[2] R. A. Albanese, R. L. Medina, and J. W. Penn. Mathematics, medicine and microwaves. Inverse Problems, 10:995-1007, 1994.

[3] R. A. Albanese, J. W. Penn, and R. L. Medina. Short-rise-time microwave pulse propagation through dispersive biological media. J. Optical Society of America A, $6: 1441-1446,1989$.

[4] J. C. Anderson. Dielectrics. Chapman and Hall, London, 1967.

[5] H. T. Banks, M. W. Buksas, and T. Lin. Electromagnetic Material Interrogation Using Conductive Interfaces and Acoustic Wavefronts, volume FR21 of Frontiers in Applied Mathematics. SIAM, Philadelphia, PA, 2000.

[6] H. T. Banks and N. L. Gibson. Electromagnetic inverse problems involving distributions of dielectric mechanisms and parameters. Technical Report of the Center for 
Research in Scientific Computation: CRSC-TR05-29, Aug., 2005, Quarterly of Applied Mathematics, to appear.

[7] H. T. Banks and N. L. Gibson. Well-posedness in maxwell systems with distributions of polarization relaxation parameters. Technical Report of the Center for Research in Scientific Computation: CRSC-TR04-01, Jan., 2004, Applied Math. Letters, to appear.

[8] H. T. Banks, N. L. Gibson, and W. P. Winfree. Gap detection with electromagnetic terahertz signals. Nonlinear Analysis: Real World Applications, 6:381-416, 2005.

[9] I. Barba, A. C. L. Cabeceira, M. Panizo, and J. Represa. Modelling dispersive dielectrics in TLM method. Int. J. Numer. Model., 14:15-30, 2001.

[10] B Bidegaray-Fesquet. Stability of fd-td schemes for maxwell-debye and maxwell-lorentz equations. Technical Report LMC-IMAG 1077-M, Laboratoire de Modélisation et de Calcul, CNRS, 2005.

[11] K. S. Cole and Cole R. H. Dispersion and absorption in dielectrics. J. Chem. Phy., 9:341-351, 1941.

[12] S. A. Cummer. An analysis of new and existing FDTD methods for isotropic cold plasma and a method for improving their accuracy. IEEE Trans. Antennas Propagat., 45:392-400, 1996.

[13] T. A. Davis and I. S. Duff. An unsymmetric-pattern multifrontal method for sparse lu factorization. SIAM J. Matrix Analysis and Applications, 1997.

[14] P. Debye. Polar Molecules. Chemical Catalog Co., New York, 1929.

[15] F. Edelvik and B. Strand. Frequency dispersive materials for 3-d hybrid solvers in time domain. IEEE Trans. Antennas Propagat., 51:1199-1205, 2003.

[16] E. C. Fear, P. M. Meaney, and M. A. Stuchly. Microwaves for breast cancer detection. IEEE Potentials, pages 12-18, 2003.

[17] J. D. Jackson. Classical Electromagnetics. John Wiley and Sons, New York, 1999.

[18] R. M. Joseph, S. C. Hagness, and A. Taflove. Direct time integration of Maxwell's equations in linear dispersive media with absorption for scattering and propagation of femtosecond electromagnetic pulses. Optics Lett., 16(18):1412-1414, 1991.

[19] T. Kashiwa and I. Fukai. A treatment by the FD-TD method of the dispersive characteristics associated with electronic polarization. Microwave Opt. Technol. Lett., 3(6):203-205, 1990.

[20] T. Kashiwa, N. Yoshida, and I. Fukai. A treatment by the finite-difference time domain method of the dispersive characteristics associated with orientational polarization. IEEE Transactions of the IEICE, 73(8):1326-1328, 1990.

[21] D. Kelley and R. Luebbers. Piecewise linear recursive convolution for dispersive media using FDTD. IEEE Trans. Antennas Propagat., 44:792-797, 1996. 
[22] Burden R. L. and J. D. Faires. Numerical Analysis. PWS, Boston, 1993.

[23] J-F. Lee, Lee Robert, and A. Cangellaris. Time-domain finite element methods. IEEE Trans. Antennas Propagat., 45:430-442, 1997.

[24] R Lee and A. C. Cangellaris. A study of discretization error in the finite element approximation of wave solutions. IEEE Trans. Antennas Propagat., 40:542-549, 1992.

[25] R. Luebbers and F. P. Hunsberger. FDTD for Nth-order dispersive media. IEEE Trans. Antennas Propagat., 40:1297-1301, 1992.

[26] R. Luebbers, F. P. Hunsberger, K. S. Kunz, R. B. Standler, and M. Schneider. A frequency dependent finite-difference time-domain formulation for dispersive materials. IEEE Trans. Electromag. Compat., 32:222-227, 1990.

[27] Peter Monk. A comparison of three mixed methods for the time-dependent Maxwell's equations. SIAM J. Sci. Stat. Comput., 13(5):1097-1122, September 1992.

[28] P. Petropoulos. Stability and phase error analysis of FDTD in dispersive dielectrics. IEEE Trans. Antennas Propagat., 42(1):62-69, 1994.

[29] K. P. Prokopidis, E. P. Kosmidou, and T. D. Tsiboukis. An fdtd algorithm for wave propagation is dispersive media using higher-order schemes. J. Electromagnetic Waves Appl., 18:1171-1194, 2004.

[30] R. Siushansian and LoVetri J. A comparison of numerical techniques for modeling electromagnetic dispersive media. IEEE Microwave Guided Wave Lett., 5:426-428, 1995.

[31] N. S. Stoykov, T. A. Kuiken, M. M. Lowery, and A. Taflove. Finite element timedomain algorithms for modeling linear Debye and Lorentz dielectric dispersions at low frequencies. IEEE Trans. Biomed. Enggr., 50:1100-1106, 2003.

[32] J. C. Strikverda. Finite Difference Schemes and Partial Differential Equations. SIAM, 2004 .

[33] D. Sullivan. A frequency-dependent FDTD method for biological applications. IEEE Trans. Microwave Theory, 40:532-539, 1992.

[34] D. Sullivan. Z-transform theory and the fdtd method. IEEE Trans. Antennas Propagat., 44:28-34, 1996.

[35] A. Taflove and S. C. Hagness. Computational Electrodynamics: The Finite-Difference Time-Domain method. Artech House, Norwood, MA, 3rd edition, 2005.

[36] J. L. Young and R. O. Nelson. A summary and systematic analysis of fdtd algorithms for linearly dispersive media. IEEE Antennas and Propagation Magazine, 43:61-77, 2001. 